RECFIVED

$\angle A-5 U B \cdot C D-T^{\prime}$

$$
\begin{aligned}
& \text { JIII } 312000 \\
& \text { Russian Academy of Sciences } \\
& \text { P. N. Lebedev Physical Institute }
\end{aligned}
$$

Фияический инстнтут им.П.Н.Лебедева РАН ФИAН

\title{
INVESTIGATION REPORT
}

Report no. 2(1995-2) File Ref. Repo95/R2-1908.doc Date Aug. 25,1995

Prepared for: Los Alamos National Laboratory

Authority: Agreement no. 8989Q0004-35, October 3, 1994

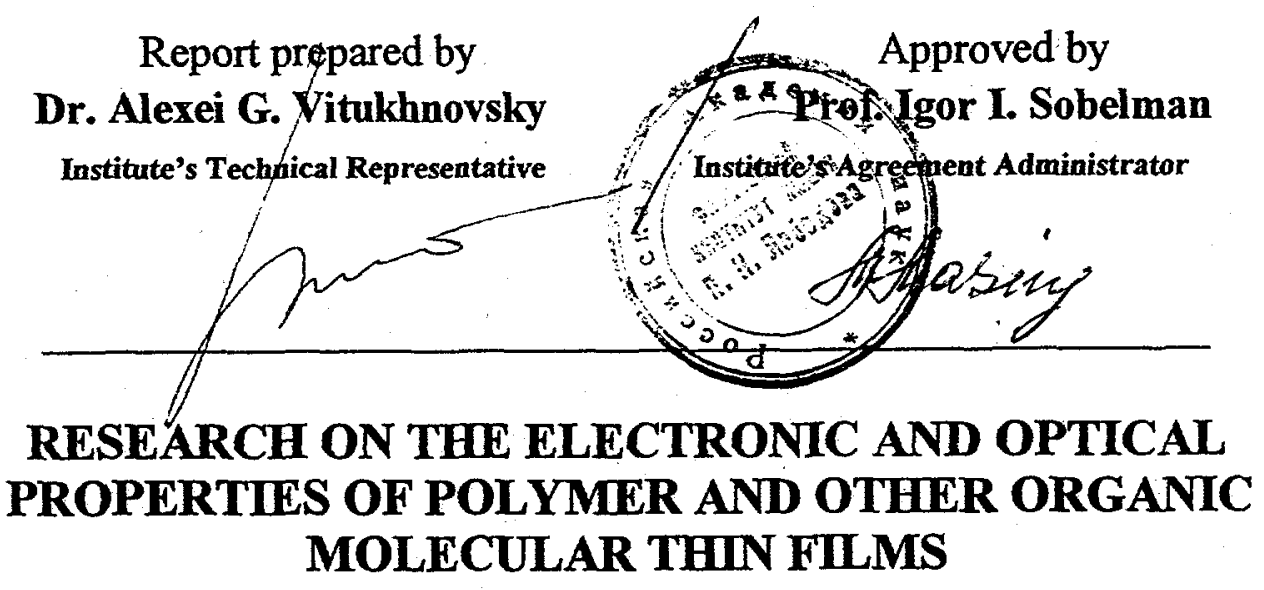

Moscow Russia

1995 


\section{DISCLAIMER}

This report was prepared as an account of work sponsored by an agency of the United States Government. Neither the United States Government nor any agency thereof, nor any of their employees, make any warranty, express or implied, or assumes any legal liability or responsibility for the accuracy, completeness, or usefulness of any information, apparatus, product, or process disclosed, or represents that its use would not infringe privately owned rights. Reference herein to any specific commercial product, process, or service by trade name, trademark, manufacturer, or otherwise does not necessarily constitute or imply its endorsement, recommendation, or favoring by the United States Government or any agency thereof. The views and opinions of authors expressed herein do not necessarily state or reflect those of the United States Government or any agency thereof. 


\section{DISCLAIMER}

Portions of this document may be illegible in electronic image products. Images are produced from the best available original document. 


\section{CONTENTS}

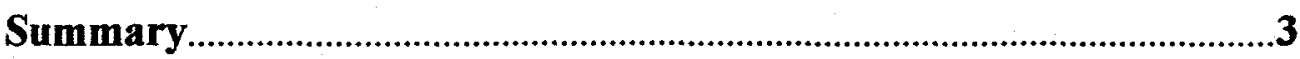

1. Introduction

2. Vacuum Deposition Prepared Poly(p-Phenylene) (PPP) Ordered Films

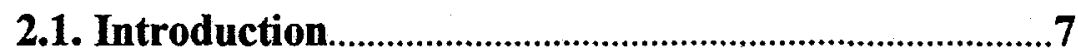

2.2. Photoluminescence Studies..............................................

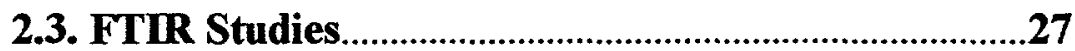

2.4. Studies of the PPP Film Crystal Structure.................31

2.5. Study of PPP Films with the Optical Microscopy....34

2.6. Selective Luminescence Spectroscopy at Low

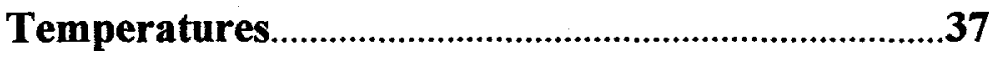

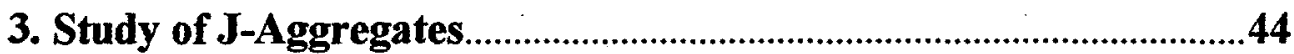

3.1. Introduction

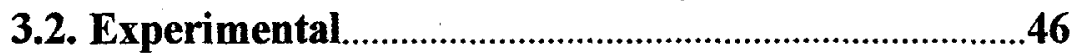

3.3. Results and Discussion.....................................................47

4. Fluorescence Properties of Polymer-Fullerene (Polyacetylene)

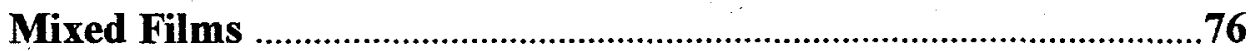

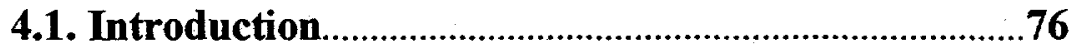

4.2. Structure and Optical Characteristics of Vacuum Deposited Fullerene $C_{60}$ Films........................................76

4.3. Electronic Structure of PPV - Fullerene $C_{60}$ Compositions .78

4.4. Electronic Structure of Fullerene $C_{60}$ - PA Composite Films..............................................................82

4.5. Electronic Structure of PPV - PA Mixtures..............87

5. Selective Laser Spectroscopy: Modern Aspects ………………........89

5.1. Introduction

5.2. Monochromatic Excitation of Luminescence of Molecules and Molecular Aggregates in Solids .90

5.3. Burning Persistent Holes in Absorption Spectra of Molecules and Molecular Aggregates............................98

6. Conclusions .108

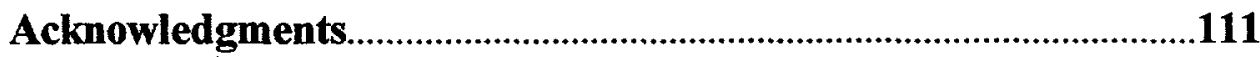

List of Researches.........................................................................111 


\section{SUMMARY}

Optical properties of highly ordered films of poly(p-phenylene) (PPP) on different substrates, thin films of mixtures of conjugated polymers, of fullerene and its composition with polymers, molecular J-aggregates of cyanine dyes in frozen matrices have been studied within the framework of the Agreement.

Procedures of preparation of high-quality vacuum deposited PPP films on different substrates (TTO, Si, GaAs and etc.) were developed. Using time-correlated single photon counting technique and fluorescence spectroscopy the high quality of PPP films has been confirmed. Dependences of structure and optical properties on the conditions of preparation were investigated. The fluorescence lifetime and spectra of highly oriented vacuum deposited PPP films were studied as a function of the degree of polymerization. It was shown for the first time that the maximum fluorescence quantum yield is achieved for the chain length approximately equal to 35 monomer units. The selective excitation of luminescence of thin films of PPP was performed in the temperature range from 5 to $300 \mathrm{~K}$. The total intensity of luminescence monotonically decreases with decreasing temperature.

Conditions of preparation of highly cristallyne fullerene $\mathbf{C}_{6}$ films by the method of vacuum deposition were found. Composites of $\mathbf{C}_{60}$ with conjugated polymers PPV and polyacetylene (PA) were prepared. Our results on fluorescence quenching, $I R$ and resonant Raman spectroscopy are consistent with earlier reported ultrafast photoinduced electron transfer from PPV to $\mathbf{C}_{60}$ and show that the electron transfer is absent in the case of the PA - $\mathrm{C}_{6}$ composition. Strong quenching of PPV fluorescence was observed in the PPV - PA blends. The electron transfer from PPV to PA can be considered as one of the possible mechanisms of this quenching.

The dynamics of photoexcitations in different types of J-aggregates of the carbocyanine dye was studied at different temperatures in frozen matrices. The optical properties of relatively simple J-aggregates with pure intrasegment relaxation, which we have found, may clarify the problem of the relationship between intrasegment and intersegment processes in the formation of luminescent states in more complicated conjugated polymers, which is important for construction of electroluminescence and photosensitive devices. 


\section{INTRODUCTION}

There is considerable scientific and technological interest in design, synthesis and characterization of organic materials that possess unusual solid state properties. Of practical interest are conjugated polymers [1] and linear molecular aggregates [2], materials in which cooperative effects have been observed.

The motivation of this research is essentially two-fold. On the fundamental level, there is a wealth of new solid state chemistry and physics to be uncovered. More practically, there is the potential for technological applications in the rapidly expanding field of molecular electronics.

As compared to widely investigated inorganic structures there are some qualitative differences which are responsible for the interesting optical and transport properties in these materials. Due to weaker intersite bonding, the electronic structure of organic systems is much less rigid than in inorganic semiconductors and changes considerably upon excitation. The strong electron-phonon interaction leads to highly nonlinear effects such as the photogeneration of solitons and polarons in conjugated polymers. A complicated interplay between the strengths of intrachain bonding, electron-electron and electron-phonon interactions in conjunction with the low dielectric constant and relatively strong disorder forms the physical picture of the elementary excitations in conjugated polymers. Because of complexity the controversy still exists in description of such excitations in conjugated polymers in terms of either of exciton or semiconductor band model predicting different processes being principal for the dynamics of the excitations. [3-6]. Recent subpicosecond fluorescence and transient absorption studies [7], fieldinduced fluorescence quenching [6] in addition to series of works on site-selective spectroscopy ([5] and references therein, this report part I) show that the primary excitations in many conjugated polymers with nongenerated ground state ( including PPV) should be excitons. In this case the associated lattice relaxation energy is relatively small $[5,7]$ On the other hand, experimental results for some structurally ordered polymer samples indicate the intrinsic energy eigenstates to be highly delocalized [4] and they can be characterized in terms of band states approach [3].

Therefore the questions what theoretical model is the most adequate one for real polymer samples (PPV,PPP etc.) and what could be the intrinsic optical and electric properties of sufficiently ordered structures are closely related to the problem of the achievable level of structural order. In the first part of this Report we described some

optical properties of highly ordered vacuum deposited films of PPP. The dynamics of excitations in such structures is of great fundamental and practical interest and we present new results on this subject in the current report. 
Optical properties of conjugated polymers are being studied in a large extend in close connection with production and transport of charge carriers. This problem is extremely important for the development of efficient light-emitting diodes (LED) and organic photodetectors as well.

Very intriguing dual function characteristic of conjugated polymer systems has been recently demonstrated: not only are organic LEDs realizable, but efficient organic photodetectors [9] and even optocouples [10] can be fabricated. The photosensitivity of some polymeric photodetectors is comparable to that of inorganic photodiodes. The photoinduced ultrafast electron transfer from the excited state of conducting polymer onto buckminsterfullerene $\mathrm{C}_{60}$ has been shown to be the key process in such photodetectors $[10,11]$. The conditions under which this process is favored are not yet clear. But it was demonstrated that for conjugated polymers with degenerated ground state (polyacetylene) the electron transfer to $\mathrm{C}_{60}$ is inhibited [12]. The search and study of new polymeric systems with photoinduced ultrafast electron transfer open up fresh opportunities in the development of new generation of organic photoelectronic devices.

There is another example of low-dimensional organic structure - molecular Jaggregates. In this system, the intersite interaction (dipole-dipole) is much less than in conjugated polymers while in many other aspects they are very similar (quasi-one dimensional structure with delocalized excitations, large disorder etc.) [2,13-15]. The elementary excitations in J-aggregates are known to be true Frenkel excitons interacting with phonons [15]. Comparing the optical excitations dynamics, "clothing" by phonons (self-trapping) in conjugated polymers and in J-aggregates one can gain a better insight into the nature of elementary excitations in conjugated polymers as well as in $\mathrm{J}$ aggregates.

In many photobiological systems, clusters of organic molecules absorb the incoming light and transport the captured energy to a reaction center, where the photonic energy is converted into a energetic intermediate [16]. Aggregates also play an important role in industry, where they are used as sensitizers for photographic materials [17] and photoconductors for electrographyc applications [18]. It was shown [2] that some aggregates exhibit a very large nonlinear optical coefficient, a feature which can be used for applications in the field of optical communications. Scientifically, aggregates are also very interesting because they bridge the gap between a single molecule and an ordered crystal.

Although a progress has been achieved in studying optical properties and energy transfer in systems with localized excitation centers, insignificant attention was paid to systems of linear chain segments, particularly, to energy transfer between chains. 
In this Report we studied the optical properties of highly ordered films of poly(pphenylene) (PPP) on different substrates, J-aggregates of the cyanine dyes in different matrices, and the mixture thin films of conjugated polymers and fullerene and polyacetylene.

Two optical experimental techniques were mainly used: time-resolved spectroscopy (nano- and picosecond time domains) and selective laser spectroscopy (fluorescence line narrowing and spectral hole burning). The method of electron diffraction, the IR-spectroscopy and optical microscopy were also used. The selective laser spectroscopy, which has been originally developed for studying impurity molecules and ions in solids, was also recently applied to investigations of polymers and molecular aggregates, however, without detailed analysis of its features. For this reason, we consider the general principles of selective laser spectroscopy of molecular systems in solids.

\section{References}

1. R.H. Friend, D.D.C. Bradley and A. Holmes, Physics World, 5 (1992) 42 N.S.Sariciftci, D.Broun, C.Zhang, V.Srdanov, A.J.Heeger, G.Stucky, and F.Wudl, Appl.Phys.Letters, 62 (1993) 585.

2. S. Kobayashi, F. Sasaki, Nonlinear Optics, 4 (1993) 305, J. Knoester, Phys.Rev.

A 47 (1993) 2083 , M.A.Drobizhev et al,Chem.Phys.Letters, 234 (1995) 425.

3. W.P. Su, J.P. Srieffer and A.J. Heeger, Phys.Rev.Letters, 42 (1979) 1698.

4. T.W. Hagler, K. Pakbaz, and A.J. Heeger, Phys.Rev.B,49 (1994) 10968.

5. U. Rauscher, H. Bassler, D.D.C. Bradley, M. Hennecke, Phys.Rev., B42 (1990) 9830.

6. R. Kersting, U. Lemmer, M. Deussen, H.J. Bakker, RF. Mahrt, H. Kurz, V.I. Arkhipov, H. Bassler, and E.O. Goebel, Phys.Rev.Lett.73 (1994) 1440.

7. R. Kersting, U. Lemmer, R.F. Mahrt, K. Leo, H. Kurz, H. Bassler, and E.O. Goebel, Phys.Rev.Lett., 70 (1993) 3820, G.S.Kanner, X.Wei, B.C.Hess, L.R.Chen, and Z.V.Vardeny, Phys.Rev.Lett., 69 (1992) 538, T. Kobayashi, Excitonic Processes in Condensed Matter, Proc. SPIE-Int. Soc . for Opt. Engin Ed. J.Singh, Darvin, 1994, Vol.2362, p. 19.

8. G. Yu, C. Zhang, A.J. Heeger, Appl.Phys.Lett., 64 (1994) 1540.

9. G. Yu, K. Pakbaz, A.J. Heeger, Appl.Phys.Lett., 64 (1994) 3422.

10. N.S. Sariciftci, L. Smilowitz, and A.J. Heeger, F.Wudl, Science, 258 (1992) 1474. 
11.K. Lee, R.A.J. Janssen, N.S. Sariciftci, and A.J. Heeger, Phys Rev.B, 49 (1994) 5781.

12. L. Smilowits, N.S. Sariciftci, R. Wu, C. Gettinger, A.J. Heeger, and F. Wudl, Phys.Rev.B, 47 (1993) 13835.

13. A.S. Davydov, Zh.Eksp.Teor.Fiz. 18 (1948) 55.

14. E.I. Rashba, Opt. Spektrosk., 2 (1957) 88.

15. H. Fidder, J. Knoester, D.A. Wịersma, J.Chem.Phys.,95 (1991) 7880.

16.G. Feher, M.Y. Okamaura, in The photosynthetic bacteria, eds.R.K.Clayton and

W.F.Sistrom (Plenum Press, New York, 1978) p.349.

17.C.H Brito Cruz, R.L.Fork, W.H.Knox and C.V.Shank, Chem.Phys.Letters 132 (1986) 341.

18.A.J. Taylor, D.J. Erskine and C.L.Tang, Chem.Phys.Letters 103 (1984) 430.

\section{VACUUM DEPOSITION PREPARED POLY(p-PHENYLENE) (PPP) ORDERED FLLMS}

\subsection{Introduction}

In the Report no. 1 [1] we showed the possibility of obtaining highly oriented PPP films using the vacuum deposition method. In this report, we thoroughly investigated the influence of the distance between initial PPP powder and a substrate on which the film is formed on the molecular structure, crystal structure and electronic excitation characteristics of vacuum deposited PPP films. According to our notations, the distance between the evaporated powder and a substrate increases linearly with decreasing film number by steps of $4 \mathrm{~mm}$ from $2 \mathrm{~cm}$ for the no. $8 \mathrm{film}$ to $4.8 \mathrm{~cm}$ for the no. 1 film. The increase in the distance between the PPP powder and a substrate results in the linear decrease in the temperature from $400^{\circ} \mathrm{C}$ for the no. $8 \mathrm{film}$ to $100^{\circ} \mathrm{C}$ for the no. 1 film

\subsection{Photoluminescence Studies}

\subsubsection{Introduction}

Optical properties of PPP films obtained by vacuum deposition from Kovacic's PPP powder have been investigated in [1]. It was found that the quality and uniformity of thin films and their fluorescence quantum yields depend on the type of a substrate and its temperature. We also detected the fluorescence quenching by the $\mathrm{Si}$ substrate. This study attempts to investigate more accurately and exactly dependencies of fluorescence spectra 
and fluorescence lifetime on the temperature of a quartz substrate and type of substrates (metal, semiconductor and dielectric). We report the results of an investigation of the effect of the substrate temperature on the length of a polymer chain and crystallinity degree of PPP films.

\subsubsection{Experimental}

Fluorescence spectra were investigated using multichannel optical analyser (EG\&G) with nitrogen laser excitation $(337 \mathrm{~nm}$ ). To investigate fluorescence spectrum of PPP films we use lens to focus laser light in a spot (less $0.5 \mathrm{~mm}$ in diameter). Fluorescence decay curves were measured with an Edinburgh Instruments spectroffuorimeter 199 by time-correlated single photon technique. The flash was a pulsed source with a repetition rate of $20 \mathrm{kHz}$ and a single-pulse duration of $1.2 \mathrm{~ns}$ full width at half maximum (fwhm). The excitation wavelength was $337 \mathrm{~nm}$. An analysis of fluorescence decay curve and experimental control were carried out with a computer. The ftuorescence decay curves were analysed by a least-squares iterative convolution method based on the Marquardt algorithm [2]. The interpretation of results is complicated by the fact that at present there is no single criterion by which the time-resolution of a fluorescence decay time instrument can be assessed. The most widely used criterion, which is adequate for most purposes if used wisely, is that of the normalised chi-squared $\left(\chi^{2}\right)$. For a good fit, $\chi^{2}$ should be "close to unity". There do not seem to be any hard rules established for the minimum number of counts required to obtain a given number of decay parameter. Often seemingly conflicting observation relates to the fact the more counts acquired in a decay the worse the $\chi^{2}$ value. The reason lies in the systematic errors becoming relatively more important than the random fluctuations of the Poissonian data. There are several sources of error: (1) the photomultiplier colour effect, (2) the monochromator colour effect, (3) the photomultiplier spatial illumination effect and (4) source instabilities. Each of them can limit time-resolution and provide spurious kinetic interpretation if not recognized and minimized. Our reconvolution software corrects influences of photomultiplier and monochromator colour effect. The linear focused photomultiplier XP2020Q which was designed for collecting photoelectrons over a relatively large photocathode area was used for overcoming spatial illumination effects. But stability cannot be guaranteed with any light source (flash-lamp and mode-locked laser). Some differential techniques which automatically correct for changes in the excitation profile offer advantages in this respect. Although differential techniques have made a considerable contribution to other branches of spectroscopy, their potential benefits have received little attention with time-correlated single photon counting. 
In order to interpret the process of fluorescence decay we use only one, two and three exponential functions for convolution. One exponential analysis is the most encountered model. It provides the most suitable model for testing the time-resolution of a fluorimeter and the level of systematic error. We tested our fluorimeter using the pterphenyl standard (fluorescence lifetime $1.2 \mathrm{~ns}, \chi^{2}=1.1$ ). Two-exponential analysis is known to be applied for many years in numerous fields. However, sometimes the decreasing the $\chi^{2}$ value when using multi-exponential analysis results only in the appearance of redundant parameters rather than in providing a complete description of the actual kinetics. Three-component analysis often has physical meaning. The decay components must be well separated to minimize correlation of the parameters. As a rule, many decay curves can be described fairly well using three-exponential law irrespective of whether or not their values have physical meaning. Hence, some knowledge of the possible photophysical behavior of the sample, but not only $\chi^{2}$ is necessary for reliable interpretation of the results. In applying three-exponential fit instead of two-exponential fit the danger of over-parameterization greater. Unfortunately, we have no more complex functions to fit the fluorescence decay (for example, to fit the Forster energy transfer kinetics).

\subsubsection{Results and Discussion}

1. Studies of dependences of fluorescence spectra and the fluorescence decay of PPP films vacuum deposited onto quartz substrates on the length of a polymer chain.

We studied eight samples deposited onto a quartz substrate. The temperature of substrates was changed linearly from $100^{\circ} \mathrm{C}$ till $400^{\circ} \mathrm{C}$. This resulted in dramatic changes of all parameters of PPP films. Investigation of the structural parameter of PPP films and details of preparation of them are described below in sections 2.3 and 2.4. Here we briefly consider the basic structural results to compare structure of PPP films with their fluorescence parameters.

FTIR studies showed that the length of phenylene chain increases with increasing substrate temperature. Fig.1(2.2) shows the dependence of the length of polymer chain ( the number of monomer units in polymer chain) on the substrate temperature. The degree of crystallinity of PPP films was investigated with the electron diffraction method. The degree of crystal order increases with increasing substrate temperature. Samples nos. from 5 to 7 have the orthorhombic crystal structure. The samples are two-dimensional polycrystals. The crystal constants are $a=7.8 \mathrm{~A}$ and $b=5.6 \mathrm{~A}$. The sample no. 8 has a poor crystal structure. The samples nos. from 1 to 4 also have a poor crystal structure. Y.Yamazaki et.al. [3] investigated the structure of vacuum evaporated thin PPP film using 
IR spectra and X-ray diffraction. These results indicate the existence of oriented single crystals in the PPP films formed on gold-coated grids $(a=7.8 \mathrm{~A}, b=5.56 \mathrm{~A}, \mathrm{c}=4.2 \mathrm{~A})$. Note that single crystals were not found in the films formed on the grids which were not coated with gold. Note that there is a good agreement between our results and [3]. For this reason, we have a good possibility for investigating photoluminescence properties depending on the length of a polymer chain and the degree of the crystal structure of PPP films deposited on quartz substrates.

Fig.2(2.2) to Fig.9(2.2) show fluorescence spectra of PPP films deposited on quartz substrates at the laser excitation at $337 \mathrm{~nm}$. These spectra strongly differ from each other. The spectra differ in: (1) positions of their maxima, (2) shapes , (3) widths, (4) and the presence or absence of the fine vibronic structure.

These figures clearly demonstrate the difference between the fluorescence spectra of different samples and the distinct dependence on the length of a polymer chain. Fig.10(2.2) shows the fluorescence excitation spectra for samples nos. 1 - 8, which were monitored at $480 \mathrm{~nm}$. One can see that the excitation spectra of different samples are different. Fig.11(2.2) and Fig.12(2.2) show the dependence of the fluorescence intensity and of the fluorescence bandwidth on the length of a polymer chain. One can see that all these dependencies exhibit the same extremum (for the 35 monomer unit). After this extremum, the fluorescente intensity and the bandwidth monotonically decrease. The fluorescence spectra of samples nos. from 5 to 7 , which are two-dimensional polycrystals, have the distinct vibronic structure (the peaks at 436,459 and $484 \mathrm{~nm}$ ). Note that fluorescence spectra of highly crystalline polyparaphenylene have no the vibronic structure $[5,6]$. It is possible that a decrease in the fluorescence intensity results from increasing number of nonfluorescent defects that quench the fluorescence.

E.M. Knapp derived the relation between the length of a molecular chain and its spectroscopic properties [4]. The theory [4] predicts that the Ionger the length of a chain, the narrower the fluorescence band. The experimental points (Fig.12(2.2)) are in agreement with [4]).

There is considerable disorder in samples of conjugated polymers, and it is the extent of the electron delocalization rather than the polymer chains that determines the optical properties. Each polymer chain is believed to consist of many conjugated segments separated by defects such as kinks in the polymer chain. We suppose that these defects of film structure do not emit. The degree of crystallinity of films increases and fluorescence spectra become more structured with increasing substrate temperature, however, the fluorescence intensity decreases because of an increase in the number of quenching defects. The sample no. 8 is not a crystal and its fluorescence spectrum is very broad, 


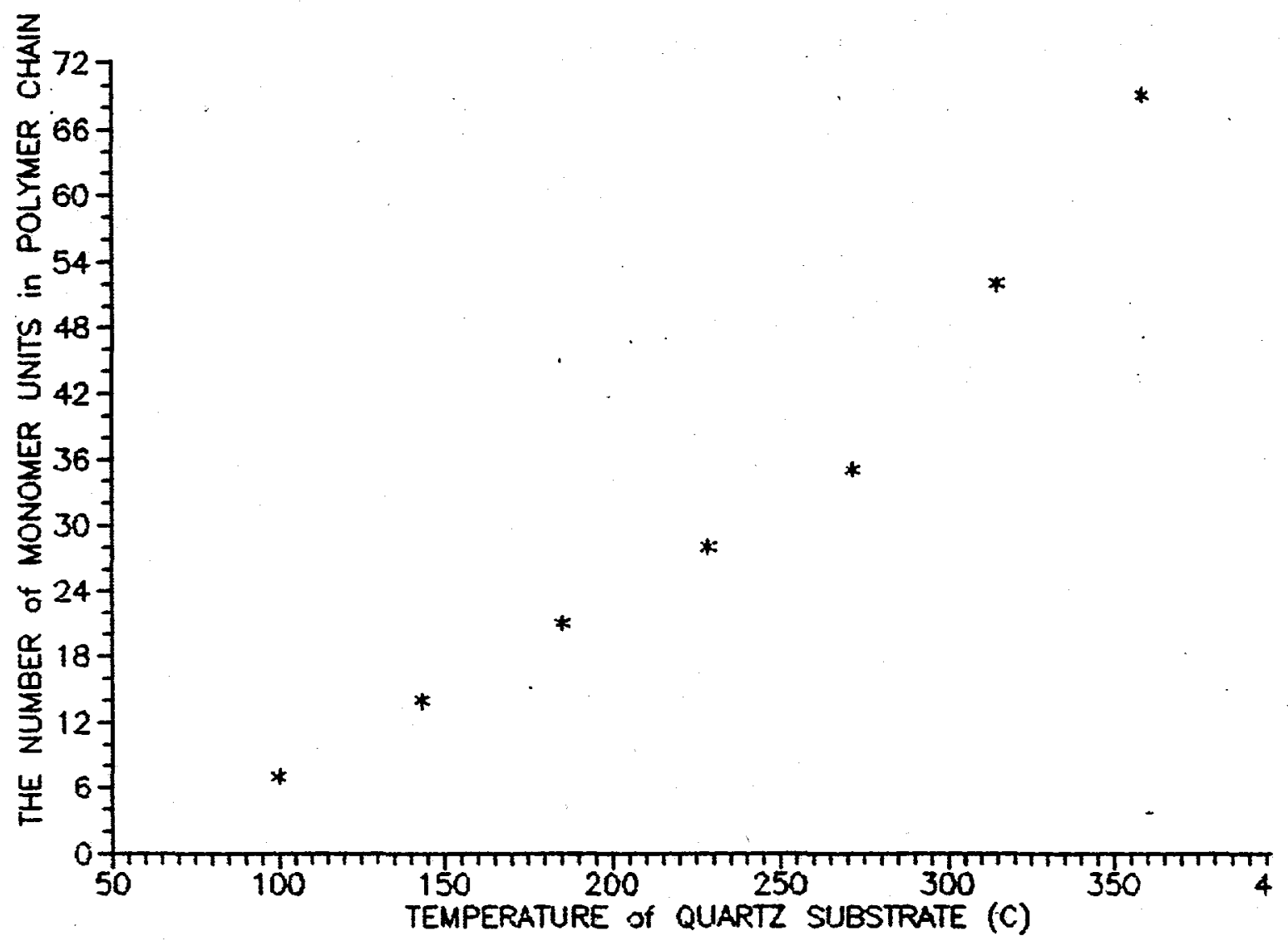

Fig.1(2.2). The dependence of length of polymer chain on temperature of substrate. 


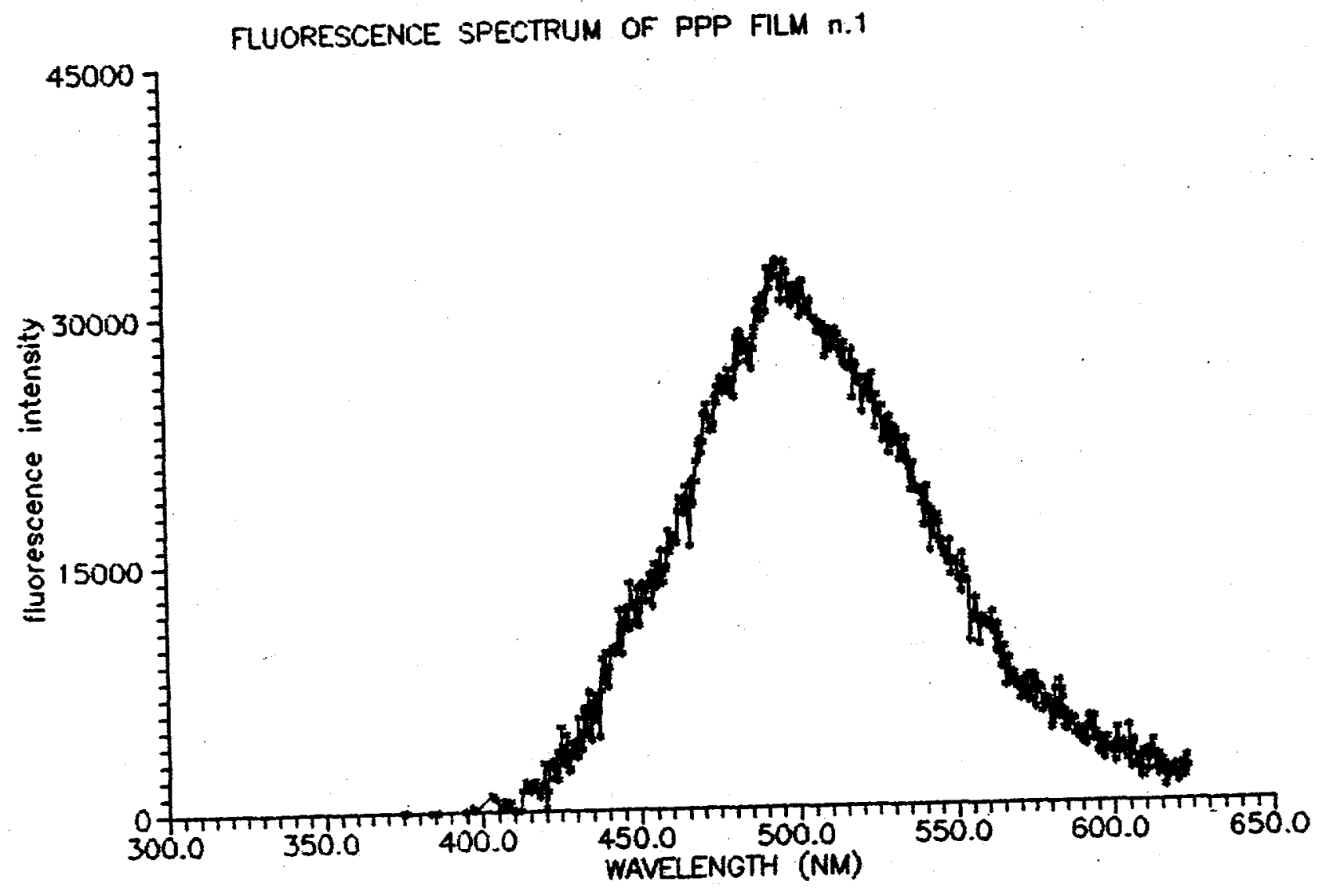

Fig.2(2.2). The fluorescence spectrum of PPP films deposited at $100^{\circ} \mathrm{C}$, sample 1, (length of polymer chain 7 monomer units). 


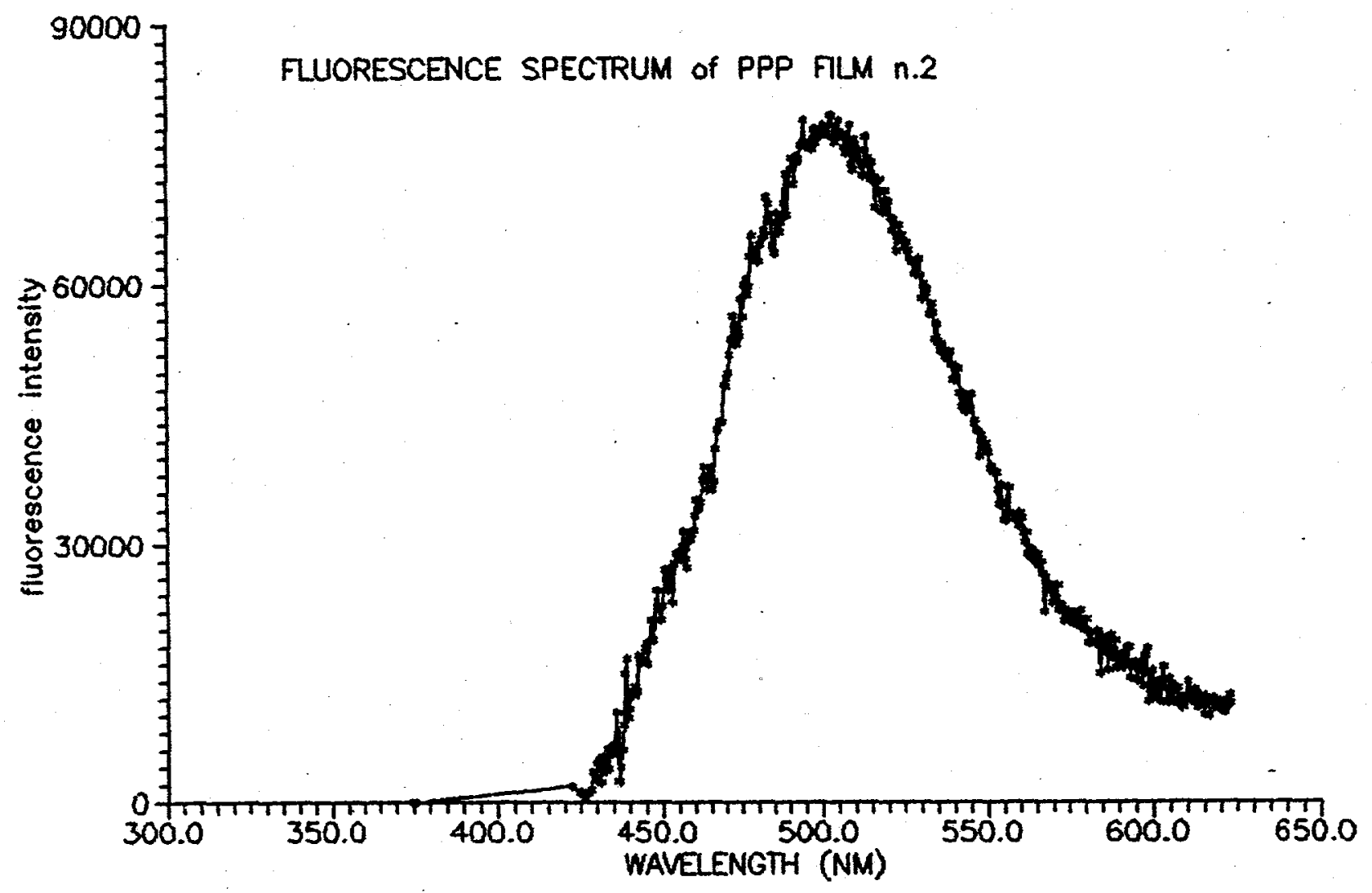

Fig.3(2.2). The fluorescence spectrum of PPP films deposited at $143^{\circ} \mathrm{C}$, sample 2 , (length of polymer chain 14 monomer units). 


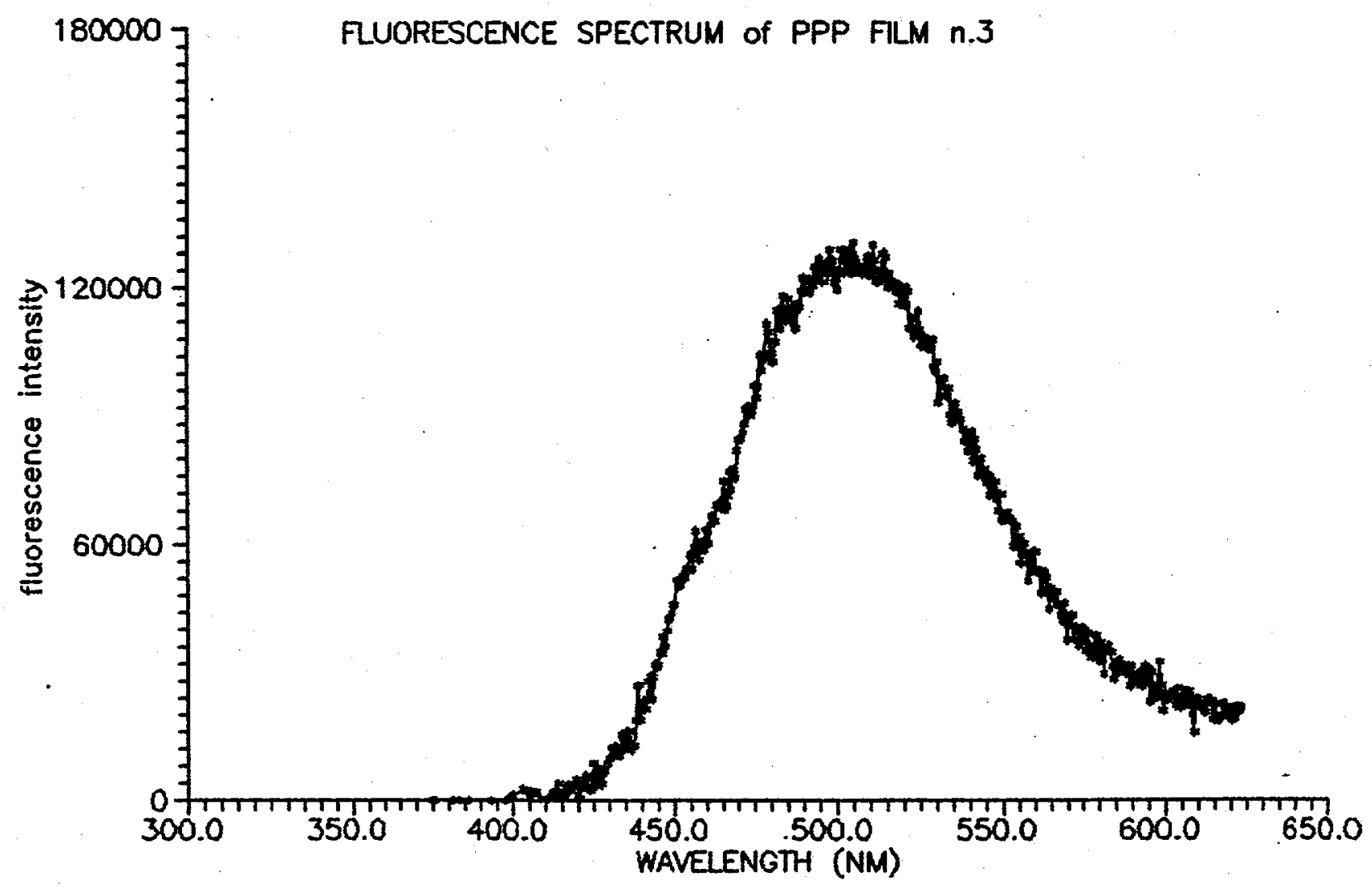

Fig.4(2.2). The fluorescence spectrum of PPP films deposited at $186^{\circ} \mathrm{C}$, sample 3, (length of polymer chain 21 monomer units). 


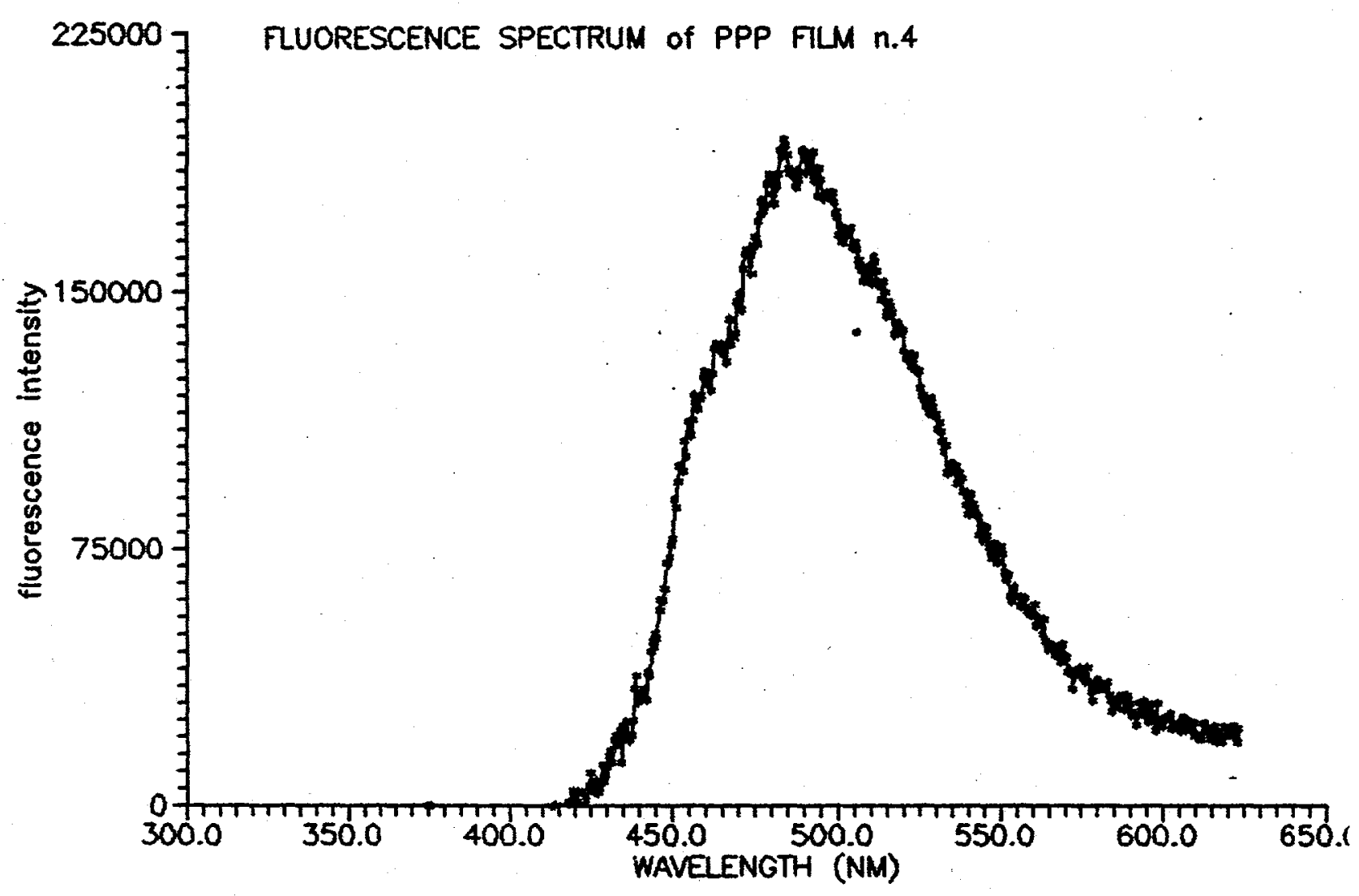

Fig.5(2.2). The fluorescence spectrum of PPP films deposited at $229^{\circ} \mathrm{C}$, sample 4, (length of polymer chain 28 monomer units). 


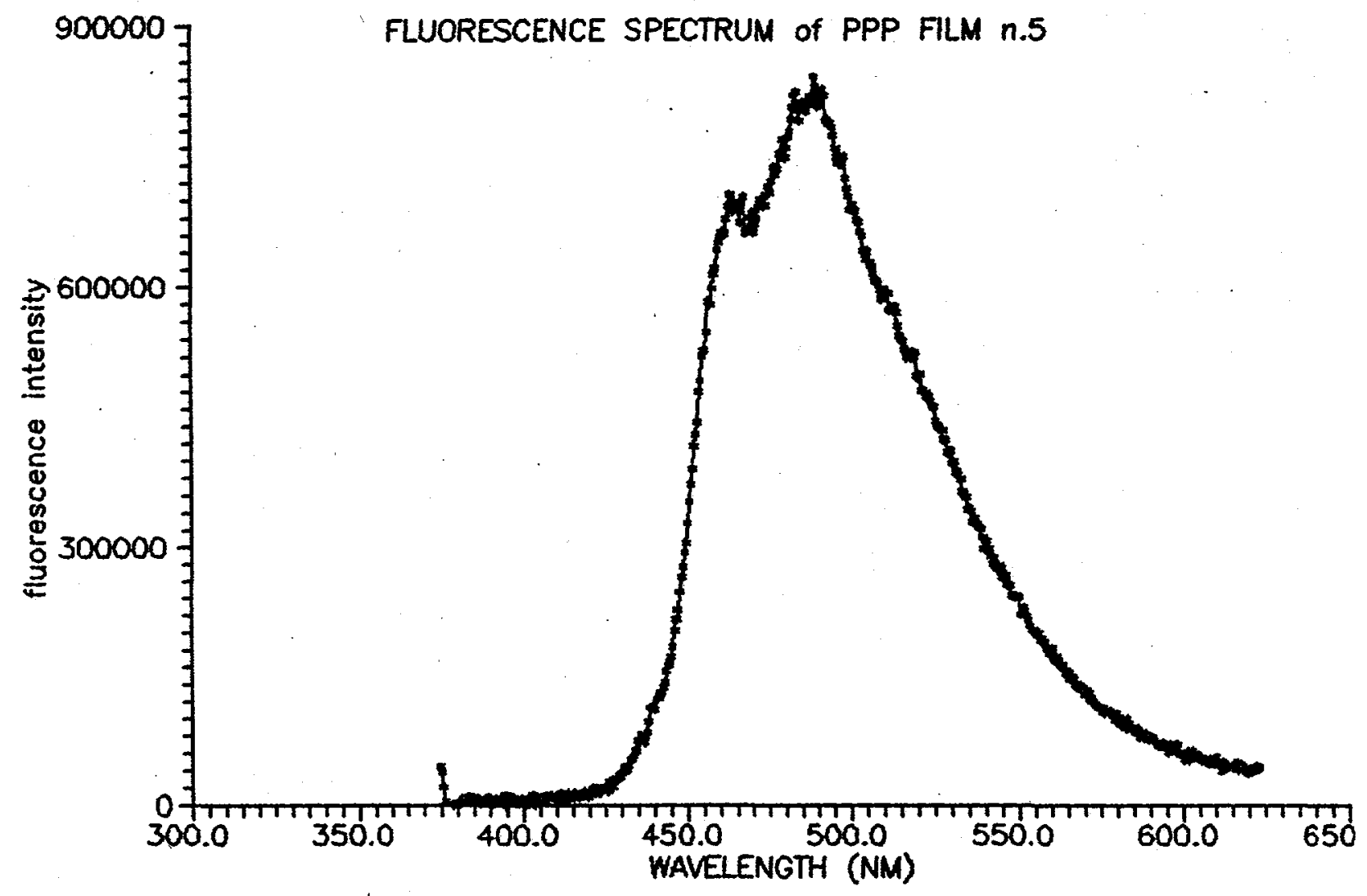

Fig.6(2.2). The fluorescence spectrum of PPP films deposited at $271^{\circ} \mathrm{C}$, sample 5, (length of polymer chain 35 monomer units). 


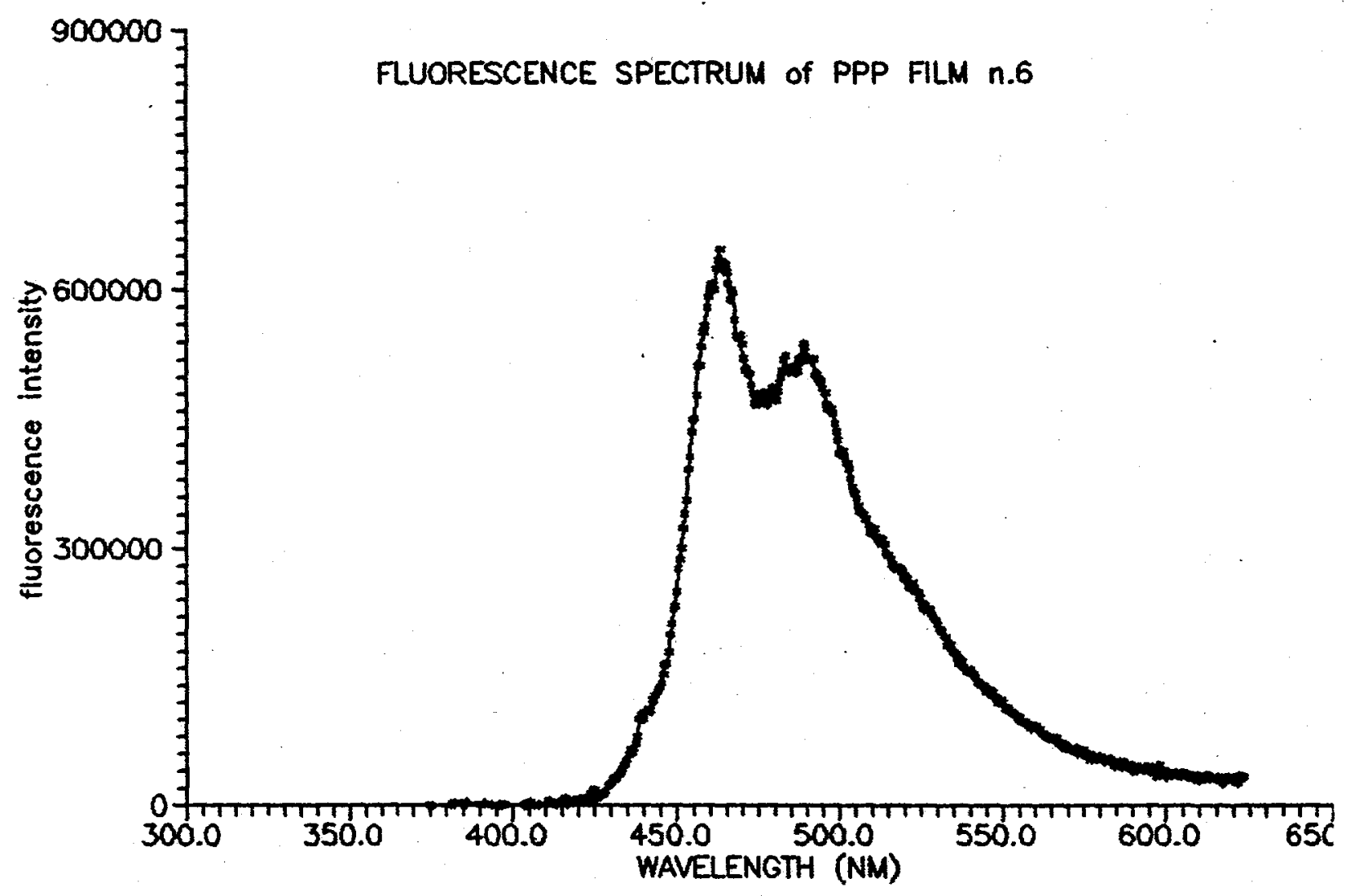

Fig.7(2.2). The fluorescence spectrum of PPP films deposited at $314^{\circ} \mathrm{C}$, sample 6 , (length of polymer chain 52 monomer units). 


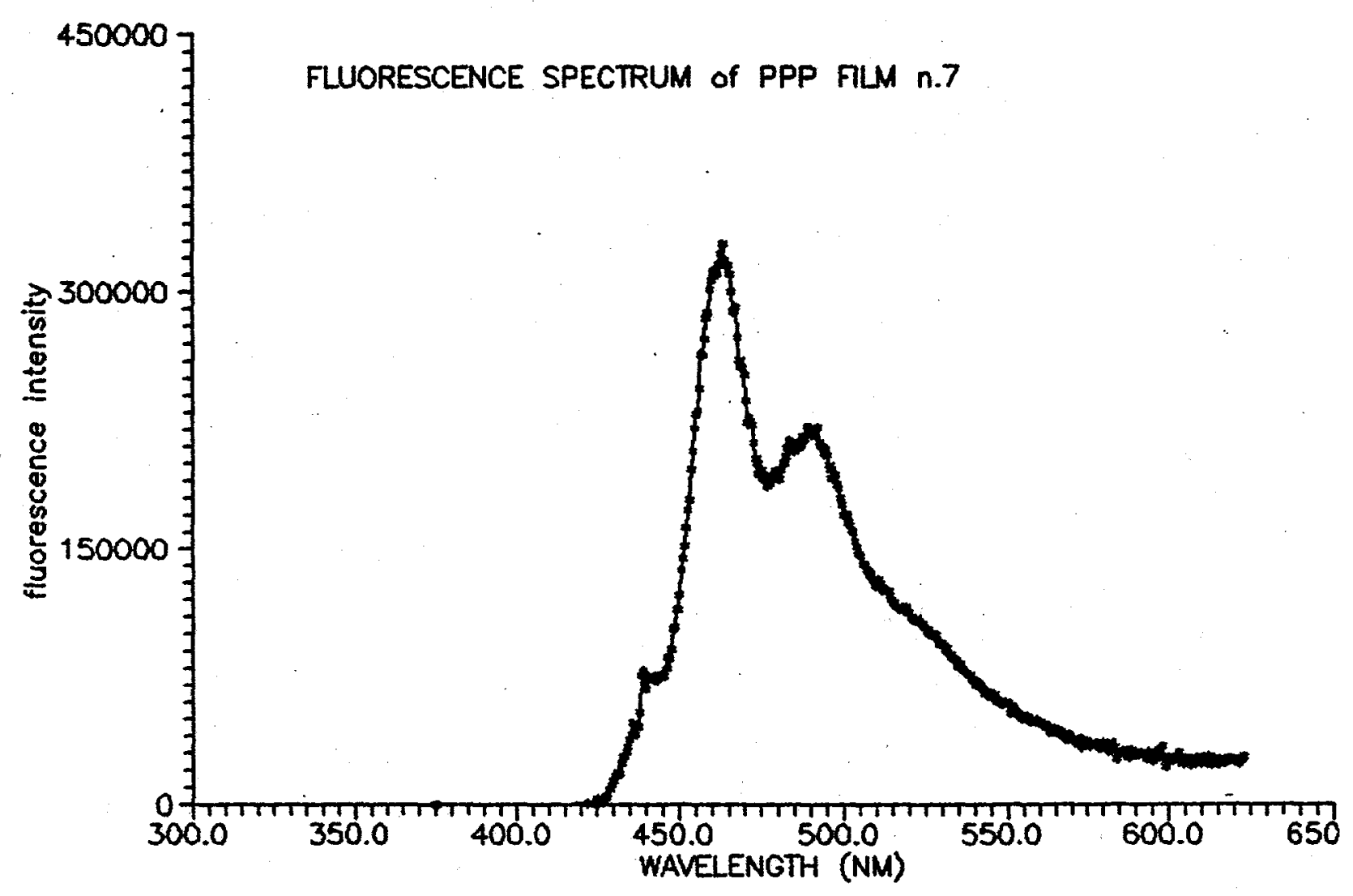

Fig.8(2.2). The fluorescence spectrum of PPP films deposited at $357^{\circ} \mathrm{C}$, sample 7 , (length of polymer chain 69 monomer units). 


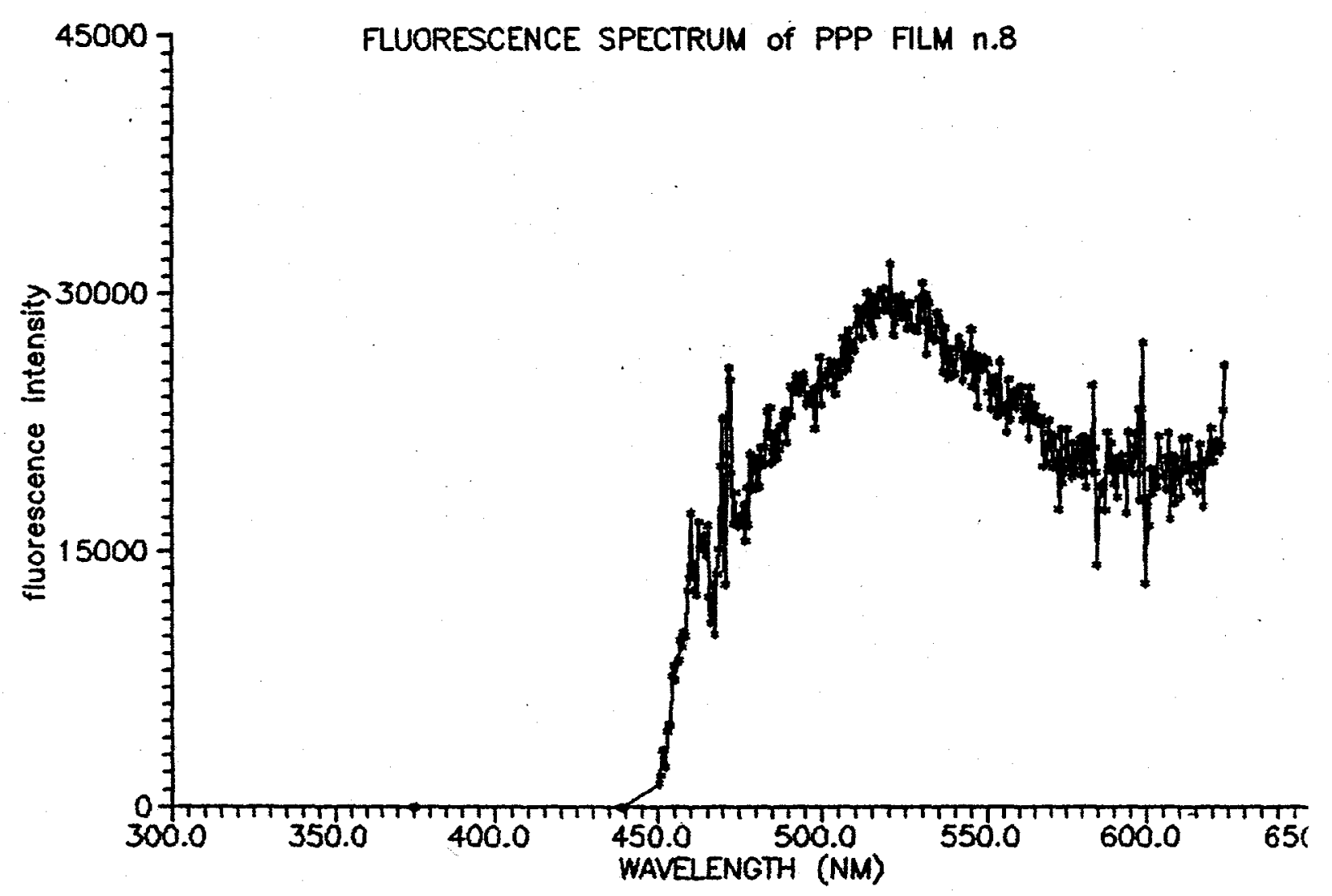

Fig.9(2.2). The fluorescence spectrum of PPP films deposited at $400^{\circ} \mathrm{C}$, sample 8, (length of polymer chain 86 monomer units). 


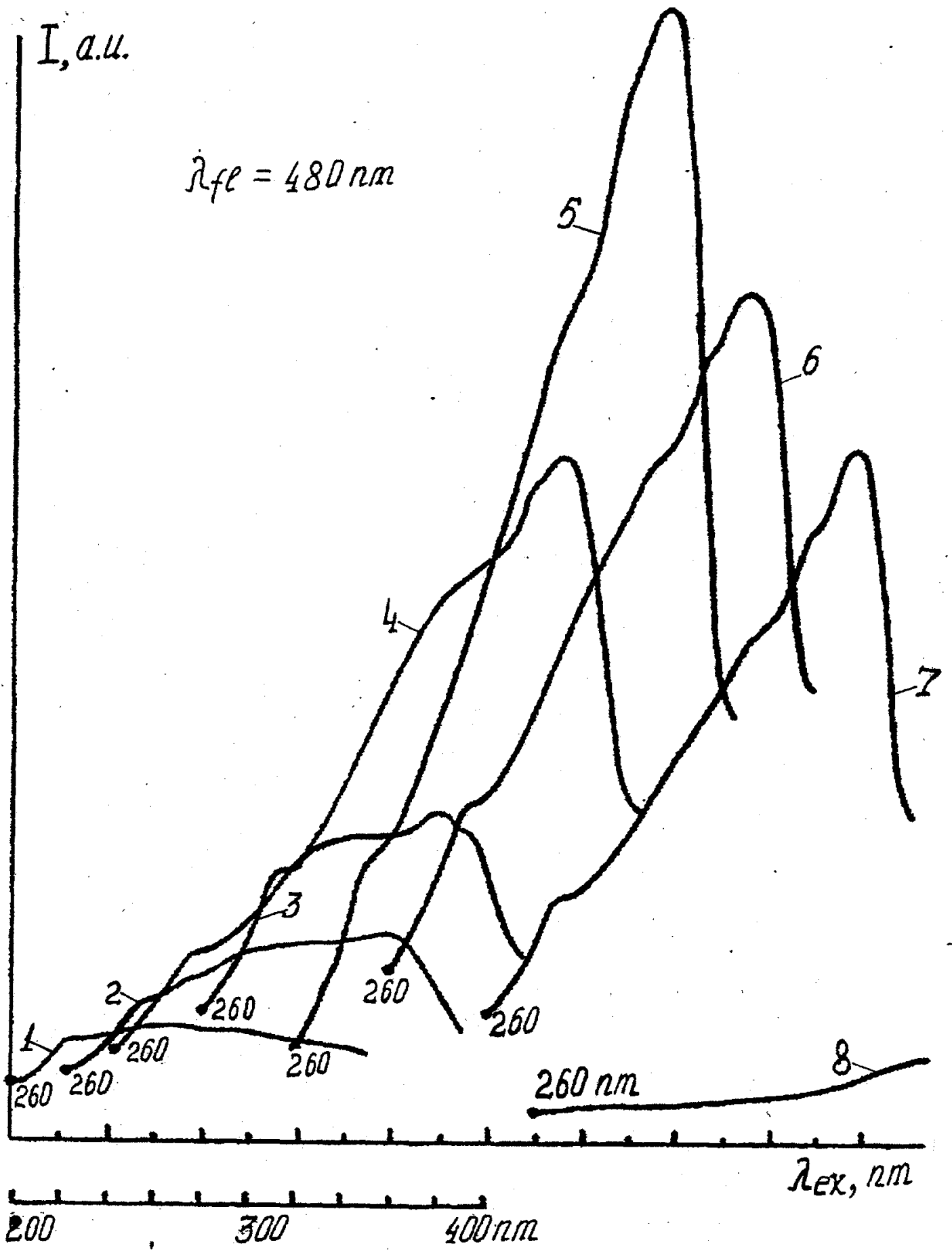

Fig.10(2.2) The excitation spectra of vacuum deposited PPP films obtained at evaporation temperature $680^{\circ} \mathrm{C}$ and distance between PPP powder and substratum from $2 \mathrm{~cm}$ for number 8 to $4.8 \mathrm{~cm}$ for number 1 with step $4 \mathrm{~mm}$ (emission - $480 \mathrm{~nm}$ ). 


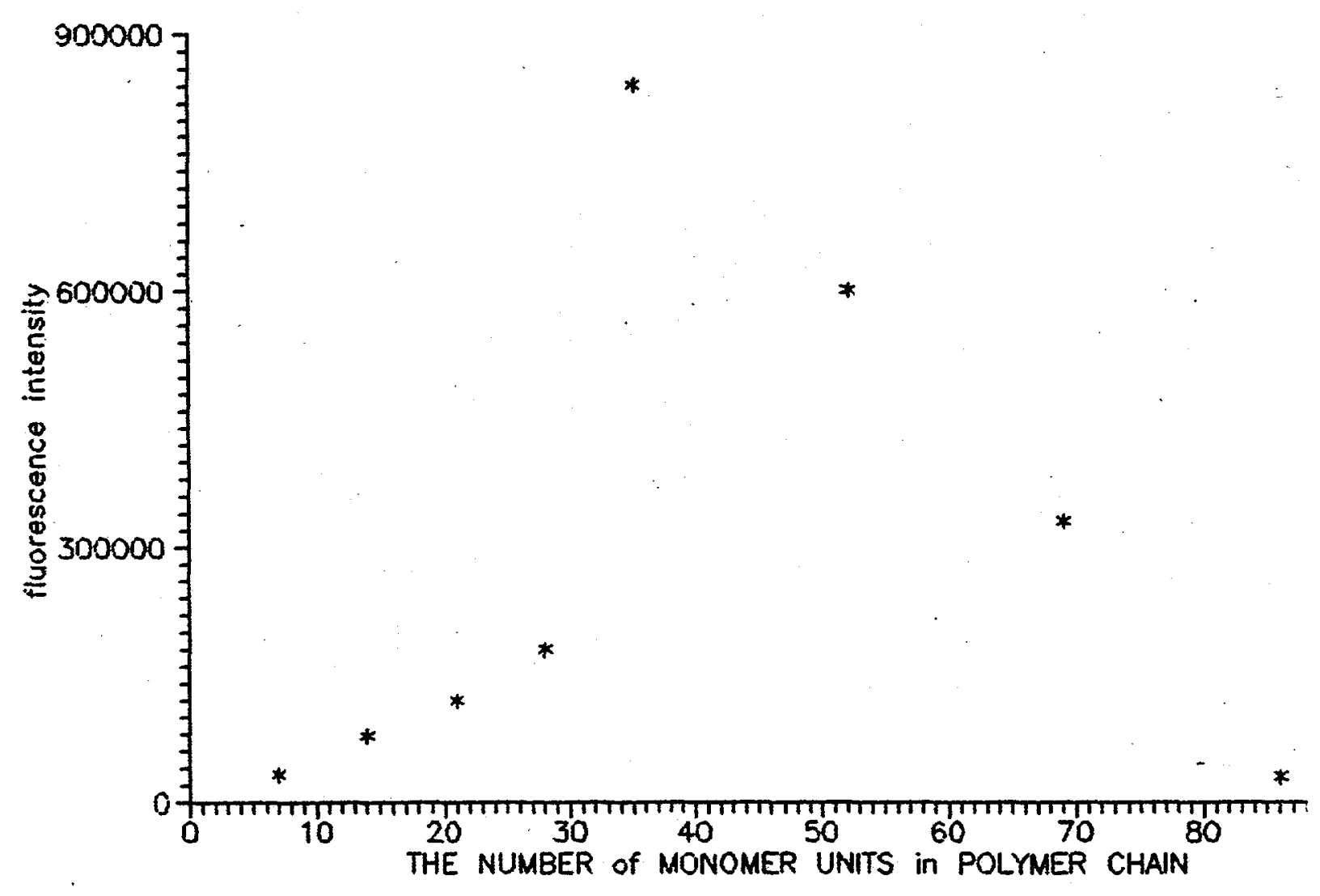

Fig. 11(2.2). Dependence of fluorescence intensity from length of polymer chain. 


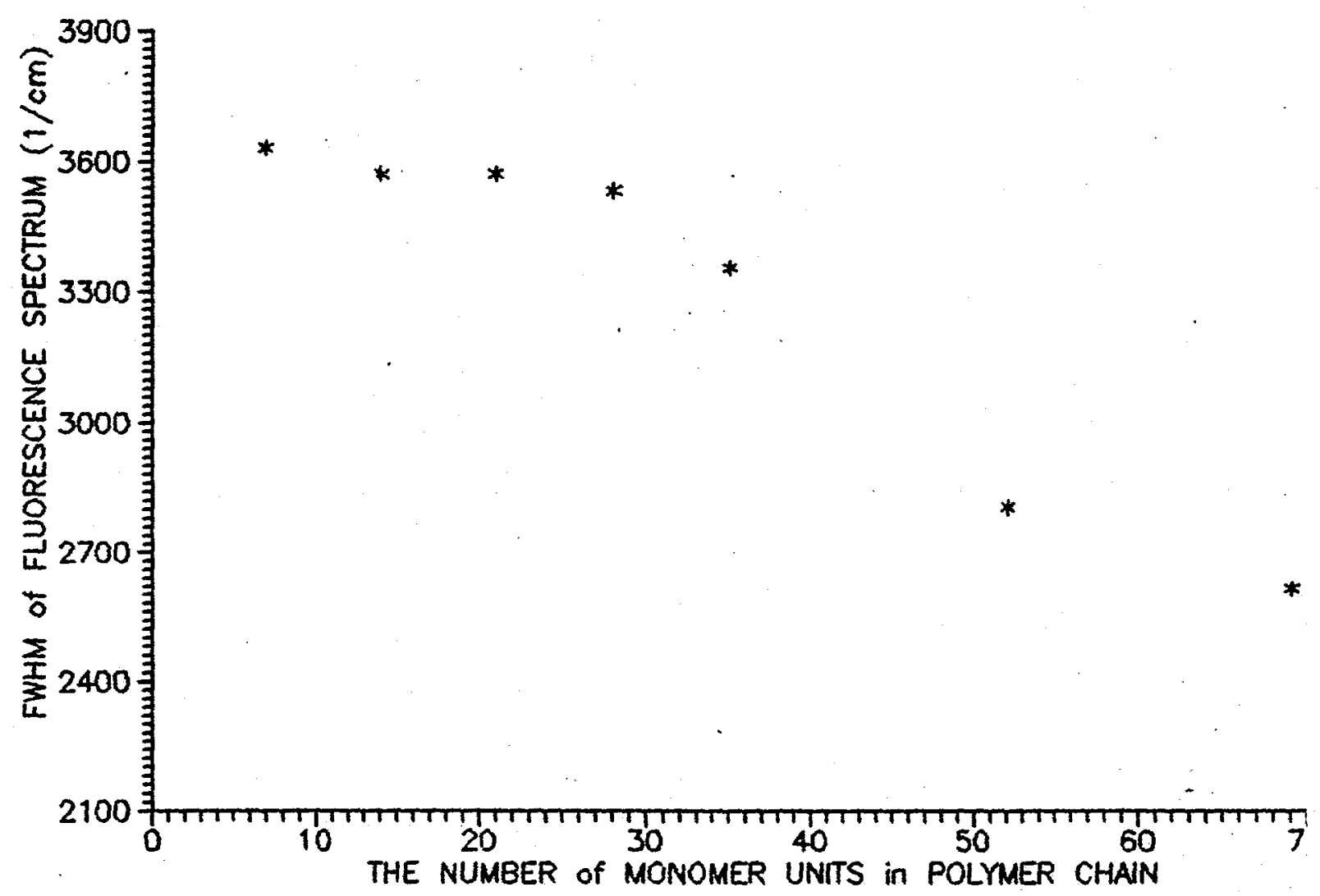

Fig. 12(2.2). Dependence of width of fluorescence band from length of polymer chain. 
shows no vibronic structure, and is shifted to the red relative to that of samples Nos. 1-7. We assume that the substrate temperature is too high, which results in the destruction of the crystal structure and in the disappearance of the vibronic structure.

Table 1 shows the fluorescence lifetime of PPP films deposited onto a quartz substrate. We obtained dependencies of the crystal structure of the PPP film and the fluorescence decay on the length of a polymer chain. The fluorescence decay was usually nonexponential because conjugated segments of polymer chains have different lengths. The decay law of samples nos. from 1 to 4 are described by two exponentials. The twoexponential decay can be related to the fluorescence of defects. If this assumption is true, the fluorescence defects will distort the fluorescence spectra. Note that fluorescent defects exist in samples with a poor crystal structure. The fluorescence lifetime virtually is independent of the length of a polymer chain. This is possibly related to the conjugated length of a polymer chain rather than to its physical length. But in crystalline samples, the fluorescence decay is described by only a single exponential. In our opinion, the value of $\chi^{2}$ is not quite good for complete interpretation of the results. We think that exponential decay is not adequate for description of the fluorescence decay in conjugated polymers. Maybe it is more acceptable to use for fitting a set of exponentials (from 1 to 100) by the maximum entropy method [7]. The distribution of coefficients $B_{i}$ in this method corresponds to the distribution of conjugated polymer lengths. Unfortunately, we have no corresponding software. But in any case, a conventional two-exponential approach gave consistent results, which are in agreement with structural investigations of PPP films.

2. Studies of dependencies of fluorescence spectra and fluorescence lifetimes of vacuum deposited PPP films on the type of substrates (metal $\mathrm{Au}$, semiconductor $\mathrm{Si}$ and GaAs, fullerene $\mathrm{C}_{60}$ )

Preparing of PPP films on different substrates is very complicated task. We do not know optimum conditions for preparing PPP films onto $\mathrm{Si}, \mathrm{GaAs}, \mathrm{Au}$ and fullerene $\mathrm{C}_{60}$ substrates. The best quality PPP films are obtained on the Si substrate. Fig.13(2.2) shows fluorescence spectra of PPP films deposited at $300^{\circ} \mathrm{C}$. Unfortunately, the substrate temperature was not measured exactly. The shape and vibronic structure of spectra are the same as that for a quartz substrate. The electron diffraction data show the crystal structure of PPP films on Si But measurements of the fluorescence decay show that there are two emitters in films. One of them has the same fluorescence decay as in the case of a quartz substrate ( $1 \mathrm{~ns}$ ), whereas the other , 4 ns. We suppose that the first emitter is related to the conjugated polymer, whereas the latter one corresponds to defects.

Fig. 14(2.2) shows the fluorescence spectrum of the PPP film deposited onto a GaAs substrate at $300^{\circ} \mathrm{C}$. The data on the fluorescence decay are presented in Table 1. 
Table 1. The best-fit parameter for fluorescence decay of PPP polymer film deposited onto different substrate. Decay law is $B 1 \exp \left(-t / t_{1}\right)+B 2 \exp \left(-t / t_{2}\right)$

\begin{tabular}{|c|c|c|c|c|c|}
\hline $\begin{array}{l}\text { The number of } \\
\text { monomer link } \\
\text { in polymer } \\
\text { chain, number } \\
\text { of sample, } \\
\text { substrate, } \\
\text { fluorescence } \\
\text { wavelength } \\
\text { (nm) }\end{array}$ & $\tau_{1}(\mathrm{~ns})$ & B1 & $\tau_{2}$ (ns) & B2 & $x^{2}$ \\
\hline $\begin{array}{c}\text { 7, n.1, quartz, } \\
490\end{array}$ & 2.3 & 0.149 & 10.1 & 0.029 & 1.8 \\
\hline $\begin{array}{c}14, n .2, \text { quartz, } \\
490 \\
\end{array}$ & 2.2 & 0.191 & 11.9 & 0.022 & 1.5 \\
\hline $\begin{array}{c}21, \text { n. } 3 \text {, quartz, } \\
490 \\
\end{array}$ & 2.6 & 0.231 & 13.2 & 0.019 & 1.8 \\
\hline $\begin{array}{c}28, n .4 \\
\text { quartz, } 460\end{array}$ & 1.3 & 0.233 & 9.8 & 0.0114 & 1.8 \\
\hline $\begin{array}{c}28, \text { n. } 4 \\
\text { quartz, } 484\end{array}$ & 1.8 & 0.232 & 10.6 & 0.0175 & 1.9 \\
\hline $\begin{array}{c}35, \text { n. } 5, \text { quartz, } \\
460\end{array}$ & 1.1 & 0.298 & & & 1.8 \\
\hline $\begin{array}{c}35, \text { n.5, quartz, } \\
484\end{array}$ & 1.4 & 0.316 & & & 1.9 \\
\hline $\begin{array}{c}52, \text { n. } 6, \\
\text { quartz, } 460\end{array}$ & 1.0 & 0.1447 & & & 1.9 \\
\hline $\begin{array}{c}52, \mathrm{n} .6 \\
\text { quartz, } 484\end{array}$ & 1.2 & 0.352 & & & 1.8 \\
\hline $\begin{array}{c}69, \text { n.7, } \\
\text { quartz, } 460\end{array}$ & 1.3 & 0.326 & & & 1.9 \\
\hline $\begin{array}{c}69, \text { n.7, } \\
\text { quartz, } 484\end{array}$ & 1.4 & 0.351 & & & 1.7 \\
\hline $\begin{array}{c}86, \text { a.8, } \\
\text { quartz, } 516 \\
\end{array}$ & 1.5 & 0.207 & & & 1.9 \\
\hline $\mathrm{Si}, 455$ & 0.9 & 0.110 & 4.1 & 0.009 & 1.1 \\
\hline $\mathrm{GaAs}, 460$ & 0.8 & 0.333 & 3.8 & 0.045 & 1.7 \\
\hline GaAs, 484 & 1.1 & 0.235 & 4.5 & 0.055 & 1.8 \\
\hline $\mathrm{Au}, 484$ & 0.5 & 0.174 & 2.1 & 0.109 & 1.7 \\
\hline $\mathrm{Au}, 500$ & 0.5 & 0.135 & 2.2 & 0.135 & 1.8 \\
\hline Fullerene, 484 & 1.2 & 0.300 & & & 1.7 \\
\hline
\end{tabular}




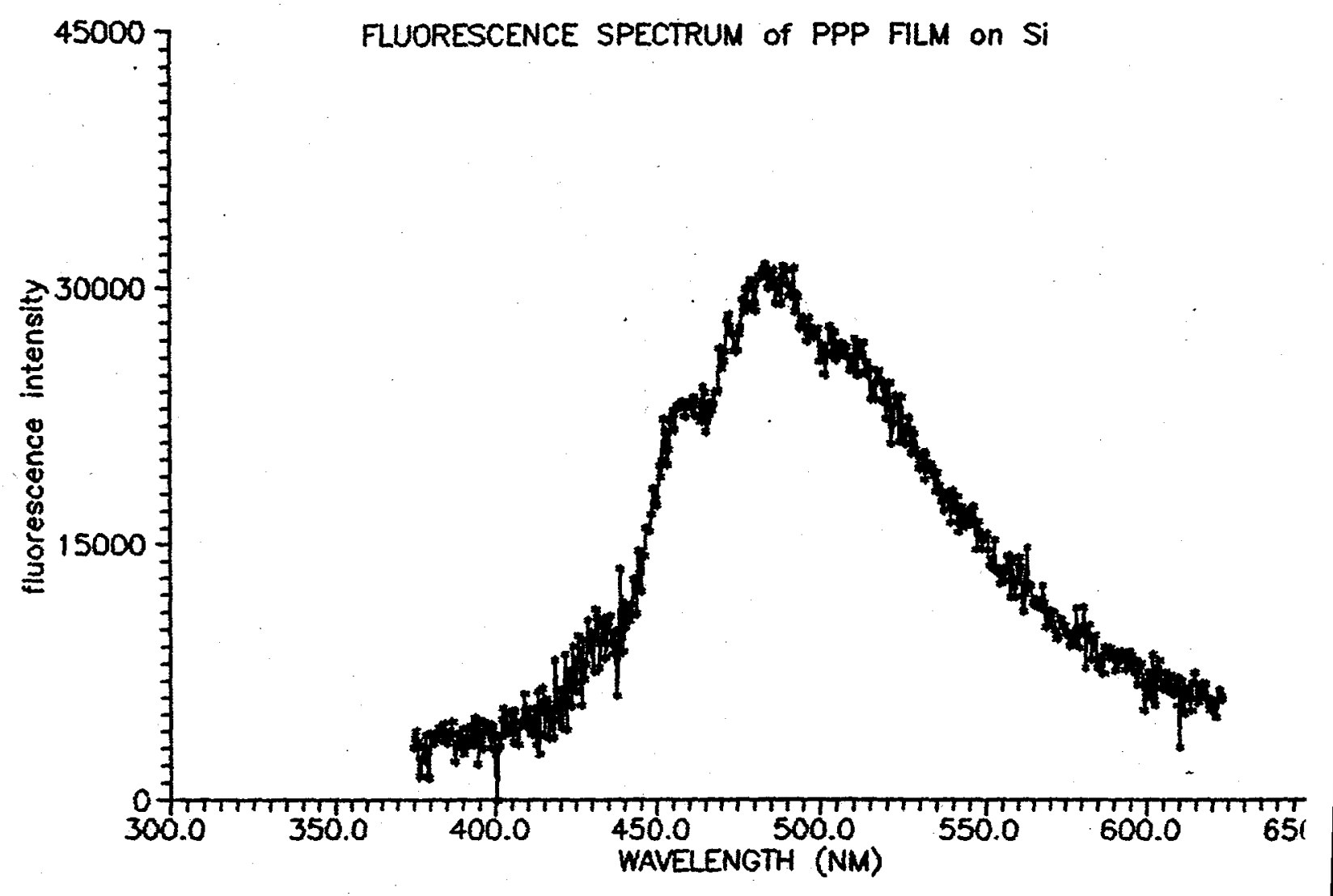

Fig. 13(2.2). Fluorescence spectra of PPP films deposited onto Si substrate (temperature of substrate is $300^{\circ} \mathrm{C}$ ). 


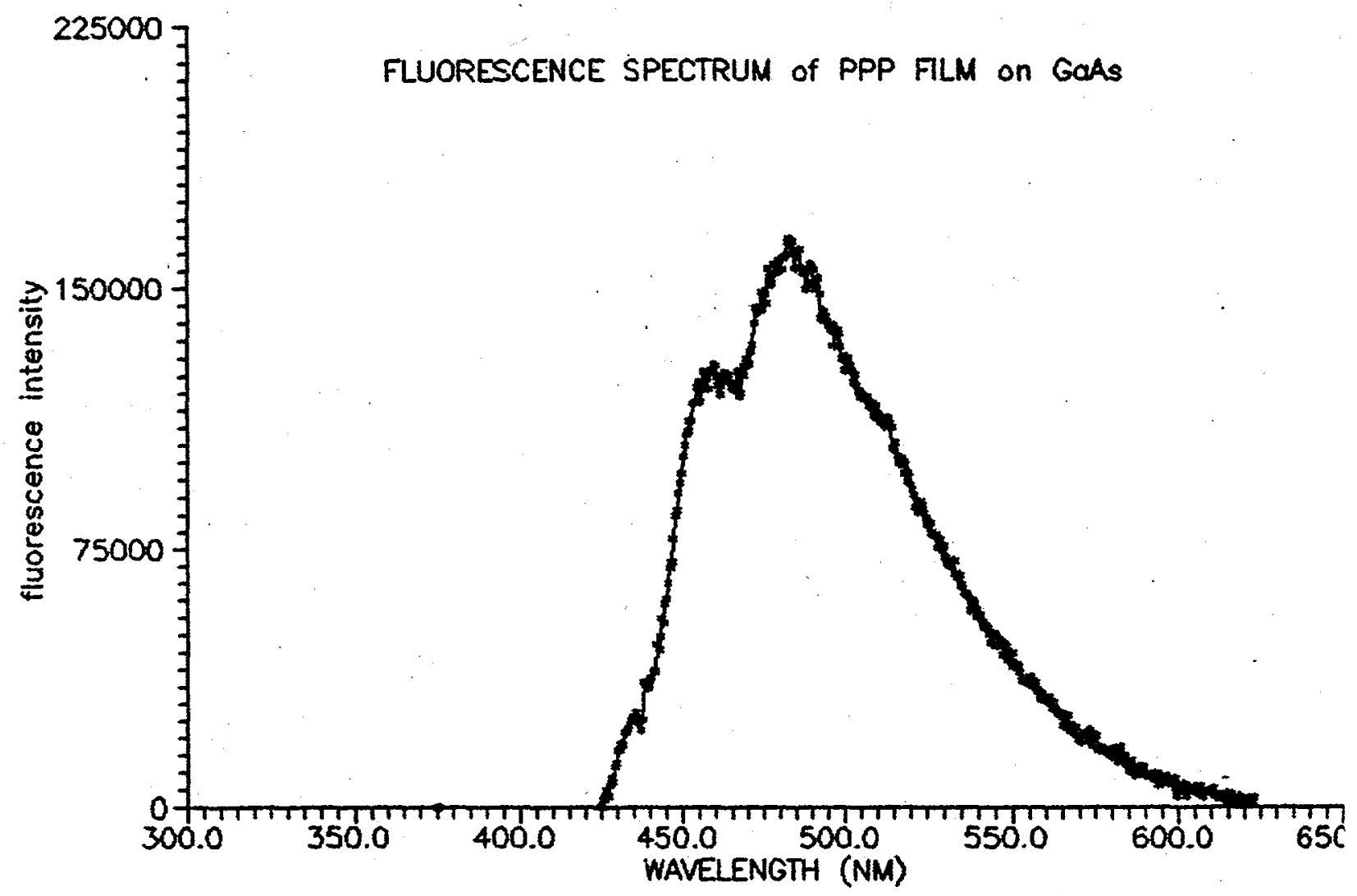

Fig.14(2.2). Fluorescence spectrum of PPP film deposited onto GaAs substrate, (temperature of substrate is about $300^{\circ} \mathrm{C}$ ). 
They are similar to those for Si substrate, being described by two exponentials and having the same fluorescence lifetimes.

Fig.15(2.2) shows the fluorescence spectrum of PPP films deposited onto $\mathrm{Au}$ substrate. Unfortunately, the spectrum does not show the vibronic structure and the fluorescence intensity is not so high as in the case of a semiconductor. This may result whether from the fluorescence quenching by a metal or from poor conditions of deposition of the PPP powder onto the Au substrate. Note that while the fluorescence decay is described by two exponentials as in the cases of Si and GaAs, the fluorescence lifetimes are smaller ( $(0.5 \mathrm{~ns}$ and $2.1 \mathrm{~ns})$. This supports the assumption about the fluorescence quenching by a metal [8].

The fluorescence quenching by Si, GaAs, and Au substrates were described within the framework of the classical treatment of energy transfer from a sensitizer molecule to a substrate. This theory considers the coupling between electric dipoles of the electronically excited sensitizer and of the substrate acceptor [8,9]. Heeger et.al [10] have established that the luminescence of MEH-PPV is quenched by fullerene $\mathrm{C}_{60}$. They proved the existence of the photoinduced electron transfer from conducting polymer to fullerene. The quantum efficiency for the charge transfer is about unity. The photoinduced electron transfer from MEH-PPV to $\mathrm{C}_{60}$ occurs on the subpicosecond time scale. Therefore, it is of particular interest to study the fluorescence of the PPP film on the fullerene layer (Fig.16(2.2)). This spectrum shows the same vibronic structure and the fluorescence lifetime as those observed for a quartz substrate. However, the fluorescence intensity is lower by a factor of 13 . This can be explained if one assumed that the fluorescence of the PPP film located near the fullerene layer is completely quenched by fullerene, so that only the fluorescence from the other side of the PPP film is observed.

\subsection{FTIR Studies}

The $\mathrm{KBr}$ substrates were prepared for measurement of IR spectra of vacuum deposited PPP films. The substrates were placed at the same distance from the PPP powder as the quartz substrate. Analysis of IR spectra allows one to determine the PPP film structure and the conjugation chain length $[1,11]$. Analysis of the IR spectra showed that PPP samples obtained on $\mathrm{KBr}$ substrate generally could be divided into two groups. The first one consists of samples no. 1 and no. 2 obtained at temperature below $150^{\circ} \mathrm{C}$. For example Fig.1(2.3) shows spectrum of the sample No. 1. The second one consists of samples obtained at higher temperatures. The spectrum that is typical for that temperature interval is shown in Fig.3(2.4) in the [1]. A comparison of the spectra in Fig.1(2.3) and Fig.3(2.4) with the spectrum of the initial PPP powder (Fig.2(2.4) of [1]) shows that 


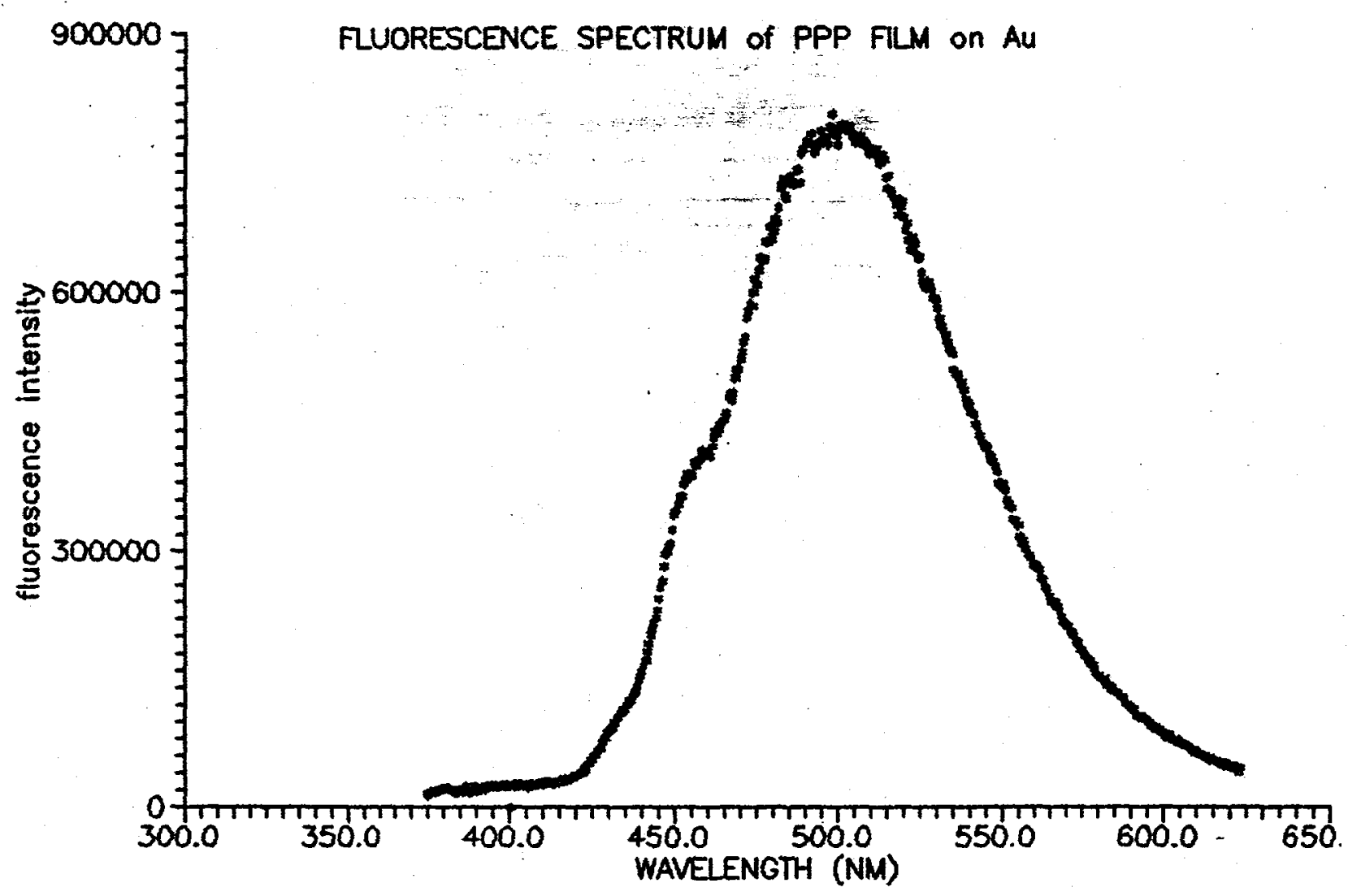

Fig.15(2.2) Fluorescence spectrum of PPP films deposited onto Au substrate. 


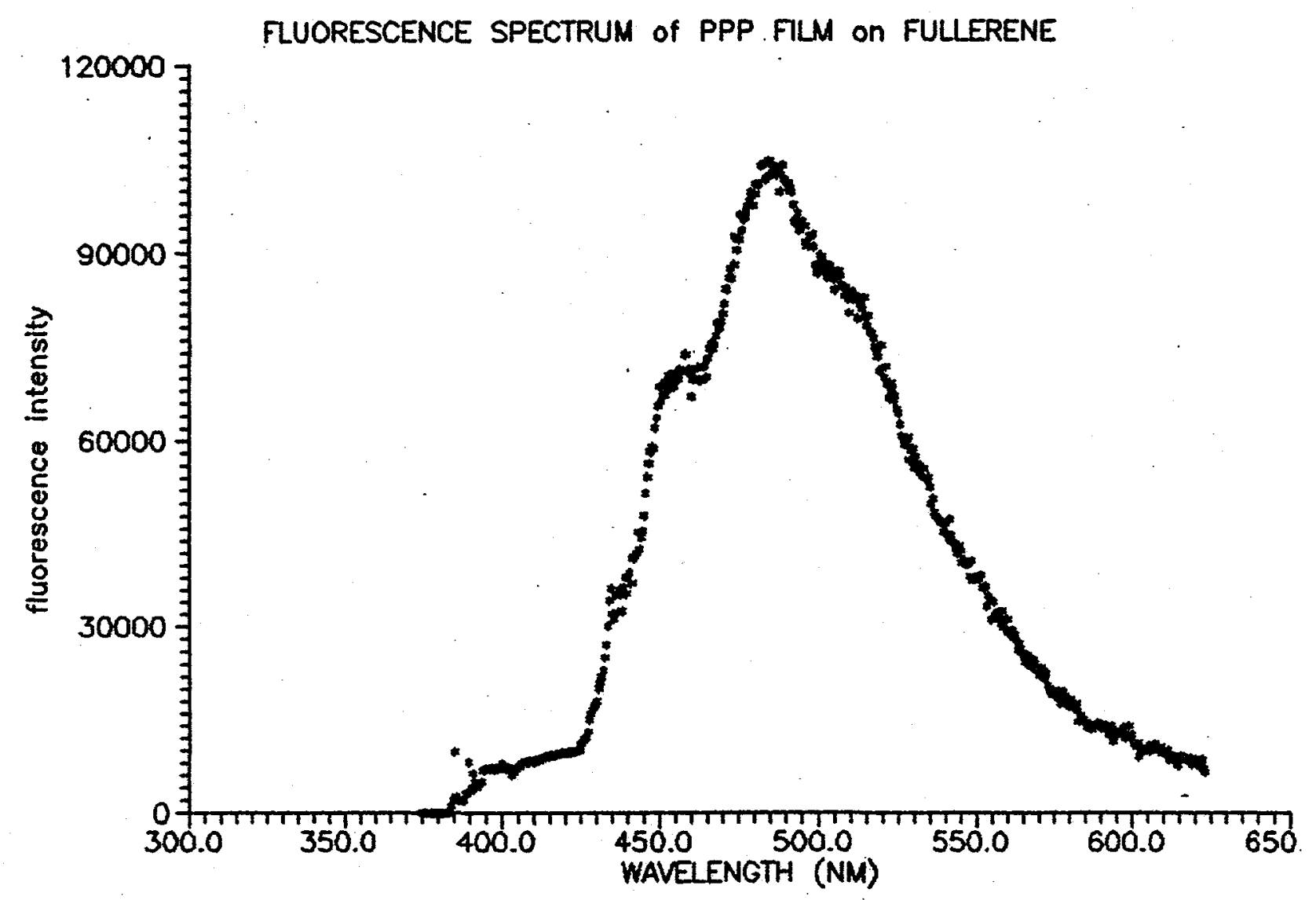

Fig.16(2.2). Fluorescence spectrum of PPP films deposited onto fullerene substrate. 


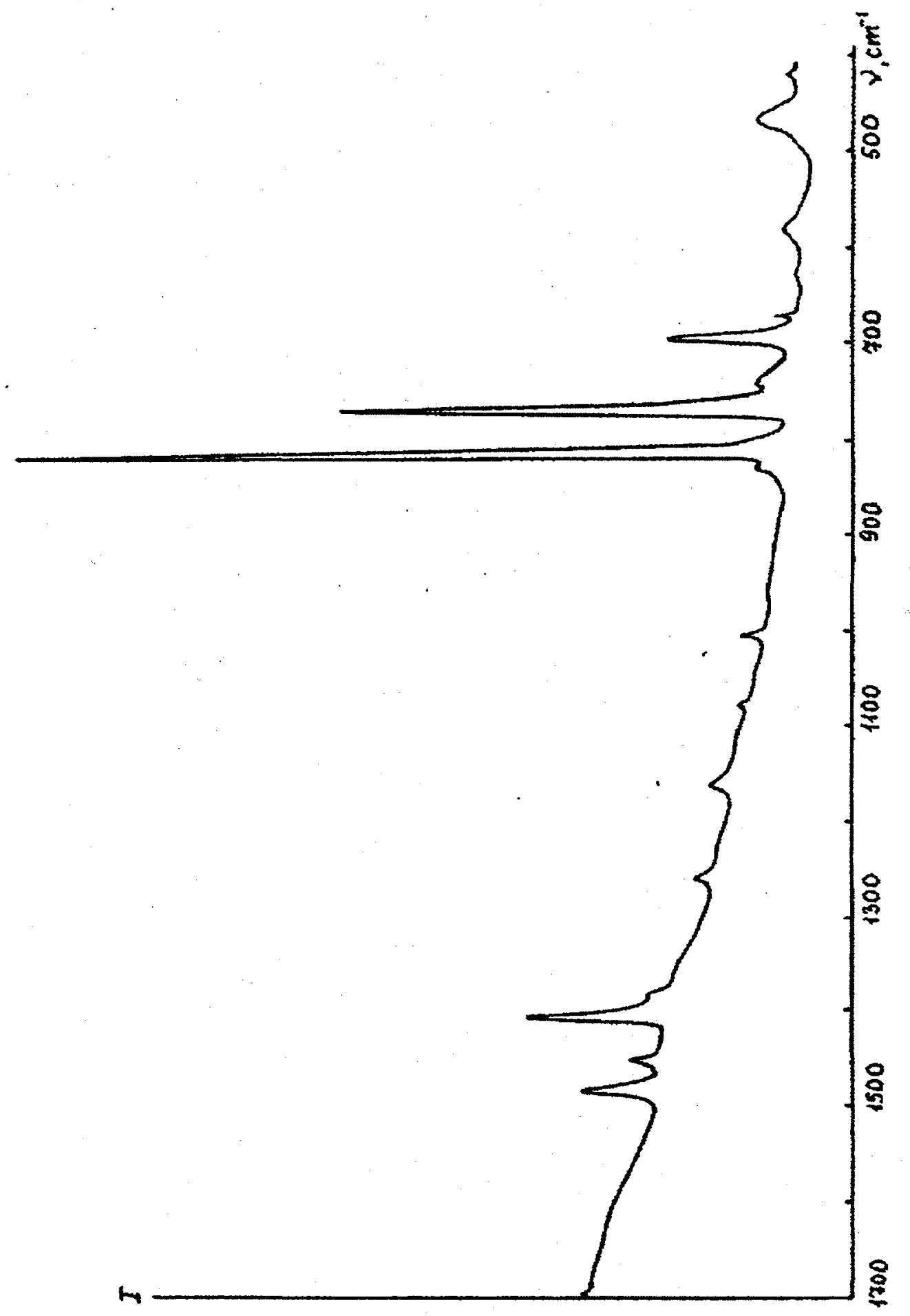

Fig.1(2.3).IR spectrum of sample number 1 . 
IR spectra of the samples obtained at low substrate temperature are very much alike the spectrum of the initial PPP powder. With increasing substrate temperature, the 1400$1375 \mathrm{~cm}^{-1}$ band intensity also rapidly increases. For PPP samples obtained at the substrate temperature $300^{\circ}-350^{\circ} \mathrm{C}$, the $1375 \mathrm{~cm}^{-1}$ band is the most intense among absorption bands in the IR spectra of PPP (Fig.3(2.4) and Fig.4(2.4) of the [1]). Probably, this is connected with the growth of the quinoid structure in PPP chains. In addition, as the temperature increases, the PPP chain length also increases. Calculations of the chain length give 6 - 8 phenyl rings for the sample no. $1,35-40$ phenyl rings for the sample no. 5 and 80-100 rings for the sample no. 8. The IR spectrum of the sample no. 8 is shown to exhibit the additional bands in the $600-1000 \mathrm{~cm}^{-1}$ region. The bands in this region characterize the type of substitution in phenyl ring and suggest the formation of intermolecular bonds at high temperature.

\subsection{Studies of the PPP Film Crystal Structure}

Investigations of the crystal structure of vacuum deposited PPP films were performed with the method of electron diffraction on an MP-102 electronograph with the accelerating voltage of $75 \mathrm{kV}$ and beam diameter of $0.2 \mathrm{~mm}$. In addition, studies were performed with a TESLA-BS-540 transmission electron microscope with the accelerating voltage of $100 \mathrm{kV}$. The crystal structure of different modifications of PPP was investigated by methods of X-ray [12-14] and electron [15-17] diffraction. Electron diffraction analysis of vacuum deposited PPP films were described in $[16,17]$. It was shown that PPP films evaporated on the carbon-coated grids gave nine electron diffraction rings [16]. Many diffaction patterns indicating single crystals were obtained in the PPP films formed on gold-coated grids [16]. The regular electron diffraction spots are taken as an indication of the formation of oriented crystalline PPP forms on carbon substrate at $150^{\circ} \mathrm{C}$ [17]. The results were explained by assuming that PPP molecules are arranged essentially perpendicularly to the surface of the substrate.

In this report, we studied transmission and reflection electron diffraction of vacuum deposited PPP films. In the latter case we used quartz and silicon substrates. It was shown that an increase in the substrate temperature results in an increase in the crystallinity of PPP films. The highest crystallinity degree was shown by films with nos. from 5 to 7. For example, Fig.1(2.4) shows the electron diffraction pattern of the film no. 6 on the carbon-coated grid taken with a transmission electron microscope with the electron beam incident perpendicularly to the substrate surface. Fig.2(2.4) shows the electron diffraction pattern of free standing film no. 6, which was transferred onto the pure

grid, taken with an electronograph with the electron beam incident perpendicularly to the 


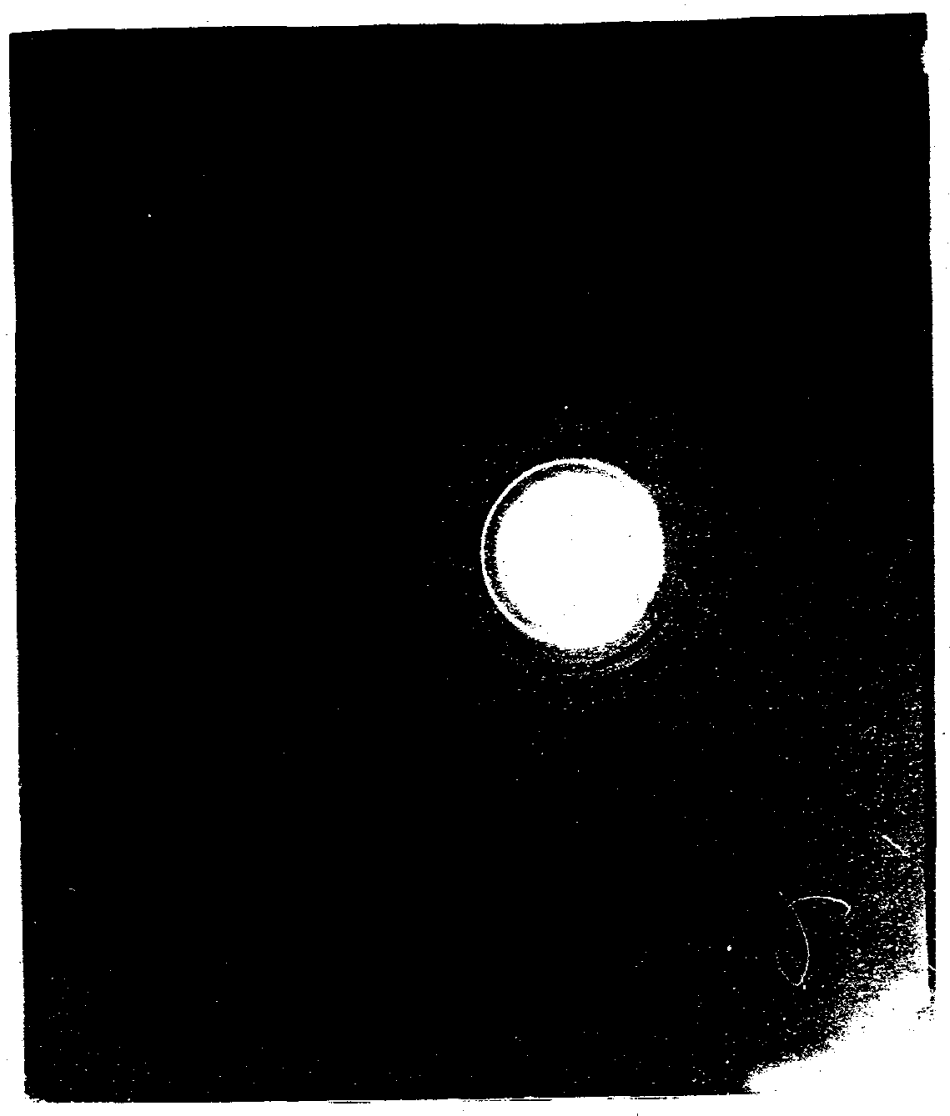

Fig.1(2.4). Transmission electron diffraction pattern of PPP film number 6 prepared on carbon-coated grid. 


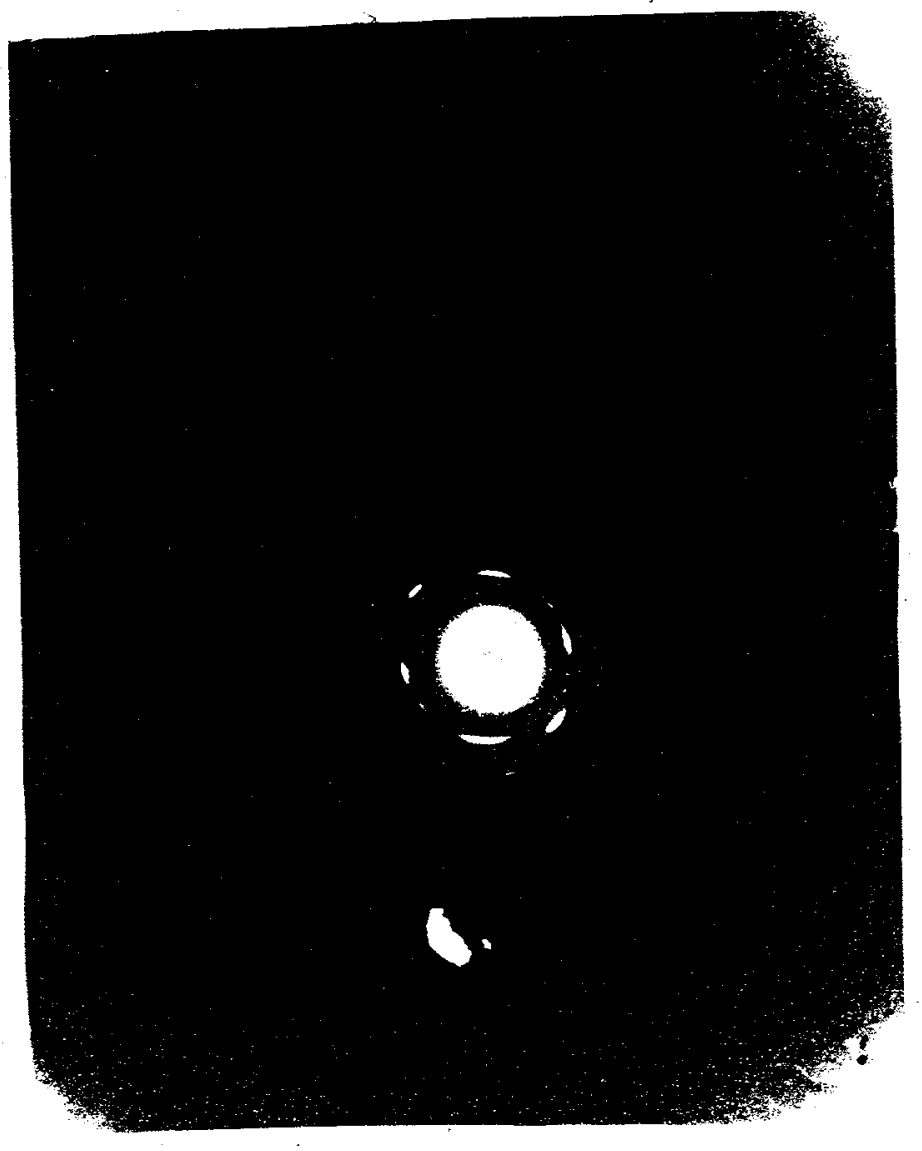

Fig.2(2.4). Transmission electron diffraction pattern of free standing PPP film number 6 prepared on cvartz substratum. 
grid surface. Unfortunately, the method of transferring of PPP films on grids did not allow to guarantee their horizontal location relative to the electron beam. For this reason we took electron diffraction patterns with different angles between the electron beam and a grid. It was shown that most crystal PPP films are formed at the substrate temperature $300-350^{\circ} \mathrm{C}$. The diffraction pattern showing spots (Fig.1(2.3) for the electron beam diameter $0.2 \mathrm{~mm}$ allows one to consider these films as high oriented two-dimensional polycrystals. The crystal orientation of PPP films and their lattice parameters were calculated from the electron diffraction data. Calculations were made by assuming that the crystal system is orthorhombic with $a=0.78 \mathrm{~nm}$ and $b=0.56 \mathrm{~nm}$. All planar distances calculated from the electron diffraction spots agree with the calculated values of parameters $a$ and $b$. These parameters are the same as those in literature $[16,17]$.

It was shown that crystal structure of vacuum deposited PPP films changes with a decrease in the substrate temperature from almost monocrystal via curved polycrystal to powder with low crystallinity. The crystal stricture of PPP films also depends on the substrate nature. Fig.3(2.4) shows the reflection electron diffaction pattern of the PPP film obtained on $\mathrm{Si}$ at $300^{\circ} \mathrm{C}$. One can see that the $\mathrm{Si}$ substrate also allows one to obtain high-oriented PPP films.

All the results obtained can be reasonably explained by assuming that at the substrate temperature $300^{\circ}-350^{\circ} \mathrm{C}$ vacuum deposited PPP molecules are arranged substantially perpendicular to the surface of the substrate.

\subsection{Study of PPP Films with the Optical Microscopy}

An MBI-15 optical microscope was used for investigation. It was shown that the transparency of PPP films transparency and their ability to scatter the light depend both on the evaporation temperature and the substrate temperature. The most transparent HT PPP films which form at the substrate temperature above $400^{\circ} \mathrm{C}$. Unfortunately, these films show very low photoluminescence intensity. The MT PPP films are highly oriented and crystalline. However, they partially scatter the light and their morphology resembles that of liquid crystal films in the electric field. Fig.1(2.5) shows the optical microscope image of the MT PPP film no. 5 taken at the magnification of 1400. Two types of fragments can be seen from the Fig.1(2.5) which are, probably, characterized by different refraction index and belong to areas with different crystallinity. 


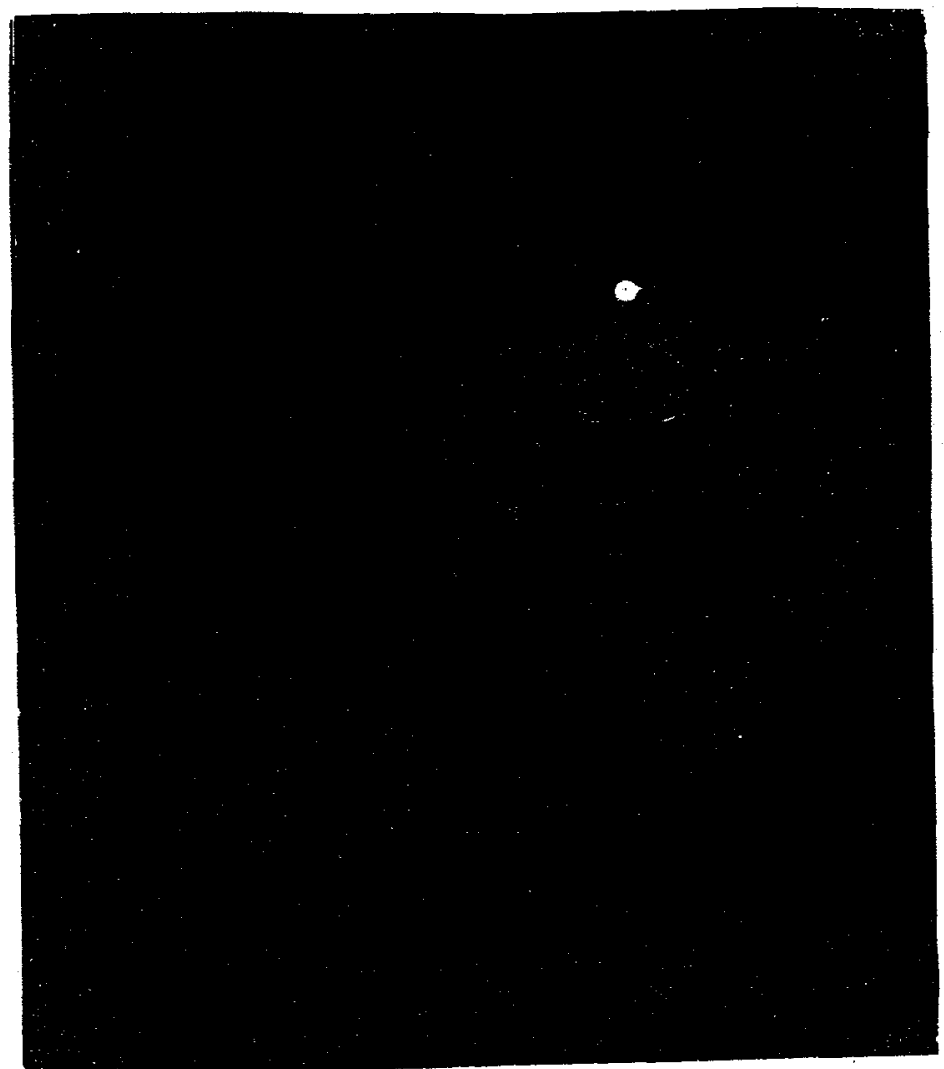

Fig.3(2.4), Reflection electron diffraction pattern of PPP film prepared on Si substratum at substratum temperature $300^{\circ} \mathrm{C}$. 


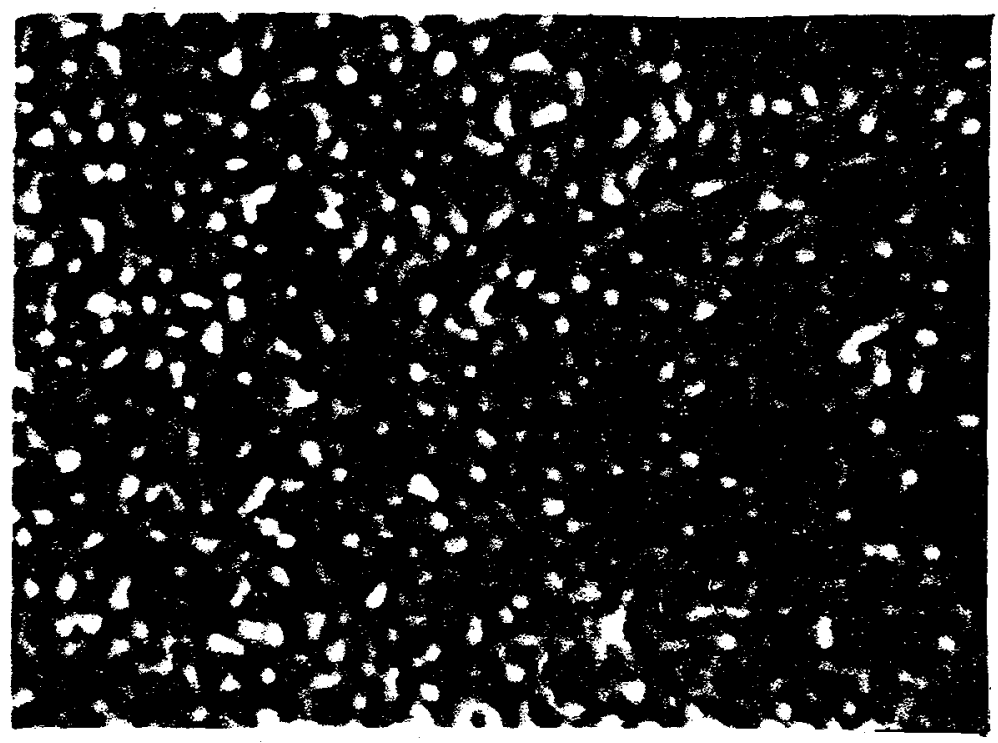

Fig.1(2.5). Optical micrograph of vacuum deposited MT PPP film number 5 taken with magnification 1400 . 


\subsection{Selective Luminescence Spectroscopy at Low Temperatures}

The luminescence spectrum of the PPP film excited by laser in the long-wavelength absorption region $\left(v_{\mathrm{cx}}<21840 \mathrm{~cm}^{-1}\right)$ reveals the following features with decreasing temperature :

(1) The total intensity of huminescence decreases monotonically (see Fig. 1(2.6)).

(2) The ill-resolved structure appears at $\mathrm{T}<200 \mathrm{~K}$ instead of the smooth red shoulder observed at room temperature (see Fig. 2(2.6)).

(3) The first intense vibronic peak exhibits the relatively narrow shoulder on its high-ferquency side at $T<80 \mathrm{~K}$.

(4) The spectral width of the transition related to this shoulder determined by computer fitting increases with temperature. However, the poor resolution of the two peaks does not allow one to infer the particular broadening law.

(5) The spectral position of the main vibronic peak is virtually independent of temperature in the range studied $(6-200 \mathrm{~K})$.

(6) Thus, the dependence of $v_{e m}$ on $v_{e x}$ at $10 \mathrm{~K}$ differs only slightly from that obtained previously [1] at room temperature for the same sample (Fig. 3(2.6)).

The first point is very surprising at the first glance. The temperature dependence of the luminescence intensity (Fig. 1(2.6)) is inversed with respect to that generally observed for molecules and polymers (e.g., PPV). But if one adopts the widely used double-well ground state potential model elaborated for $p$-terphenyl molecule $[18,19]$, this behavior becomes clear. Indeed, $p$-terphenyl molecule, which can be considered as a parent molecule for PPP, has non-planar configuration in the ground state with the internal phenyl ring twisted from the plane of the two end phenyl rings. At high temperatures, the molecules are statistically planar. In the excited state, the molecules are planar at all temperatures. Therefore, because p-terphenyl molecules are stabilized at low temperatures in one of the two valleys of the double-well potential, the absorption oscillator strength is limited by a small Frank-Condon factor. In this model, the luminescence oscillator strength is independent of temperature. The huminescence intensity is proportional to the absorption oscillator strength multiplied by the luminescence quantum yield. Because the luminescence intensity of PPP increases with temperature (Fig. 1(2.6)) in the same way as the absorption coefficient of p-terphenyl [20], we can suppose that the quantum yield is virtually independent of temperature at least for the excitation frequency used. Therefore, we can apply the following expression for the luminescence intensity

$$
F=F_{0}+A \exp (-E / k T)
$$




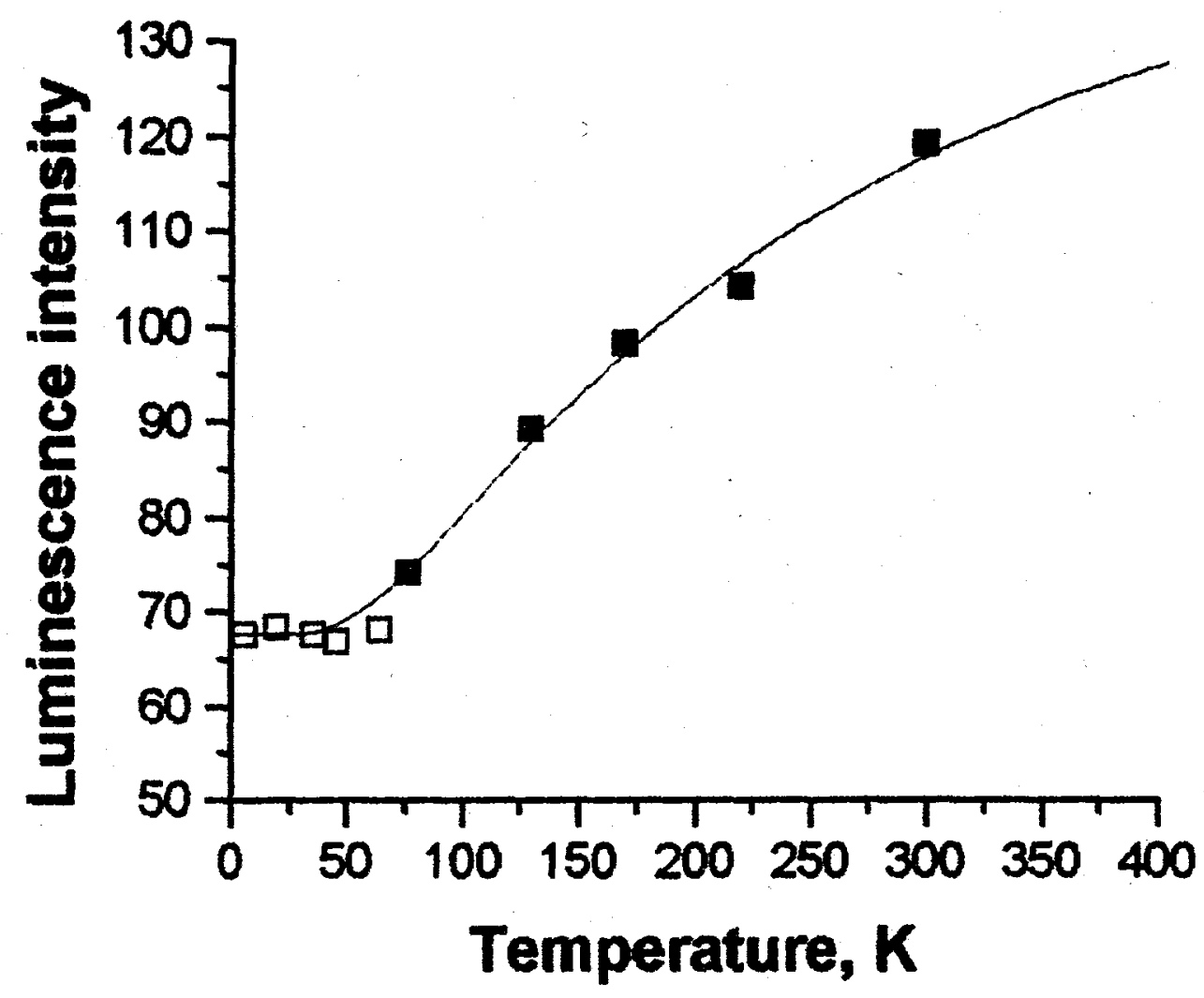

Fig.1(2.6). Temperature dependence of the maximum intensity of luminescence spectrum obtained upon laser excitation at $457.9 \mathrm{~nm}$ (full squares). The full line is the computer fitting according to Eq.(1) with $F_{0}=68, A=100$ and $E=140 \mathrm{~cm}^{-1}$. Open squares correspond to the low temperature data, obtained at excitation wavelength of $476.5 \mathrm{~nm}$ and normalized to coincide with the low temperatures limit $\mathbf{F}_{0}$. 


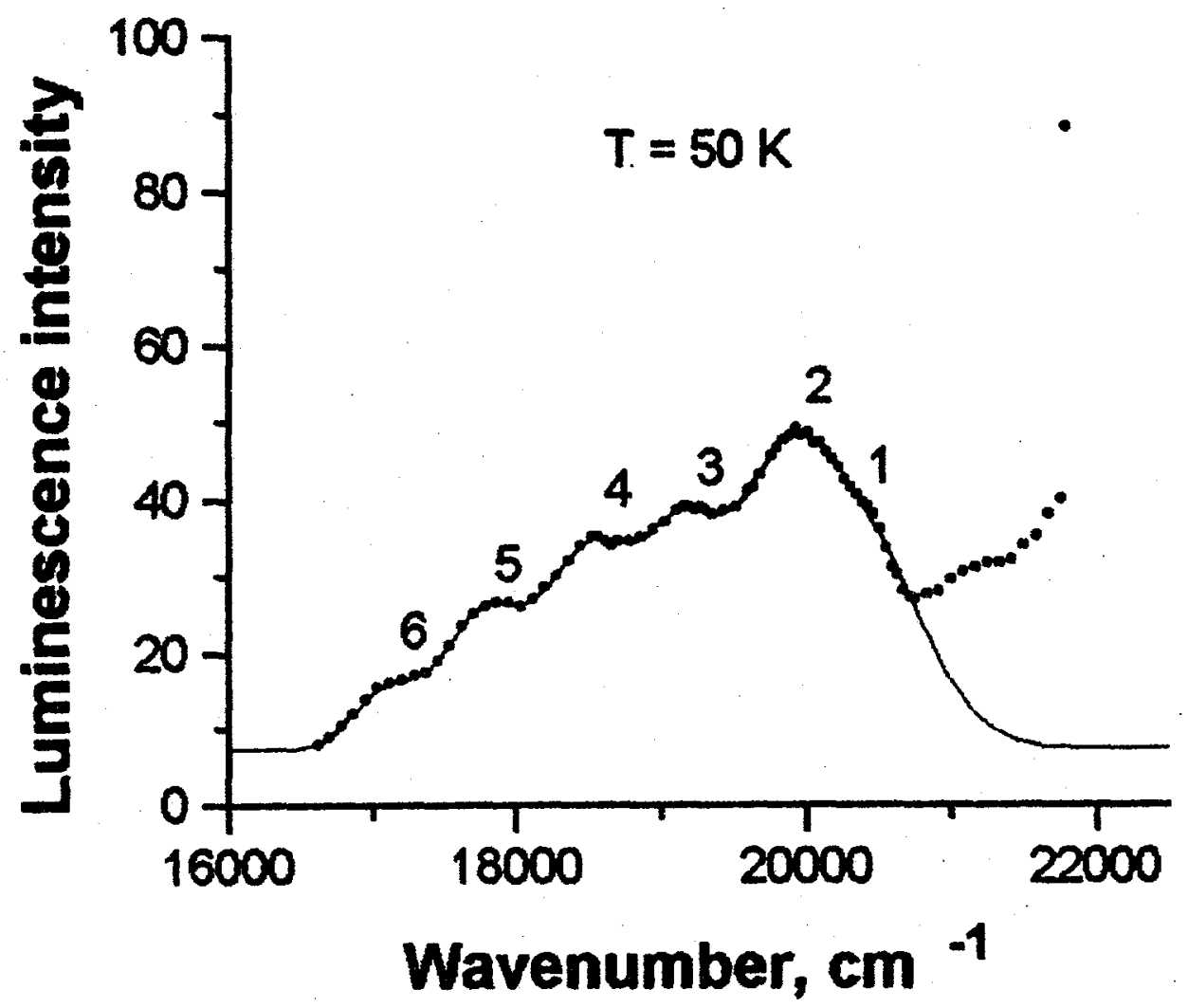

Fig.2(2.6). The low-temperature luminescence spectrum, obtained upon laser excitation at $21839 \mathrm{~cm}^{-1}(457.9 \mathrm{~nm})$ (points). The computer simulation by six Gaussian is shown (full line).

39 


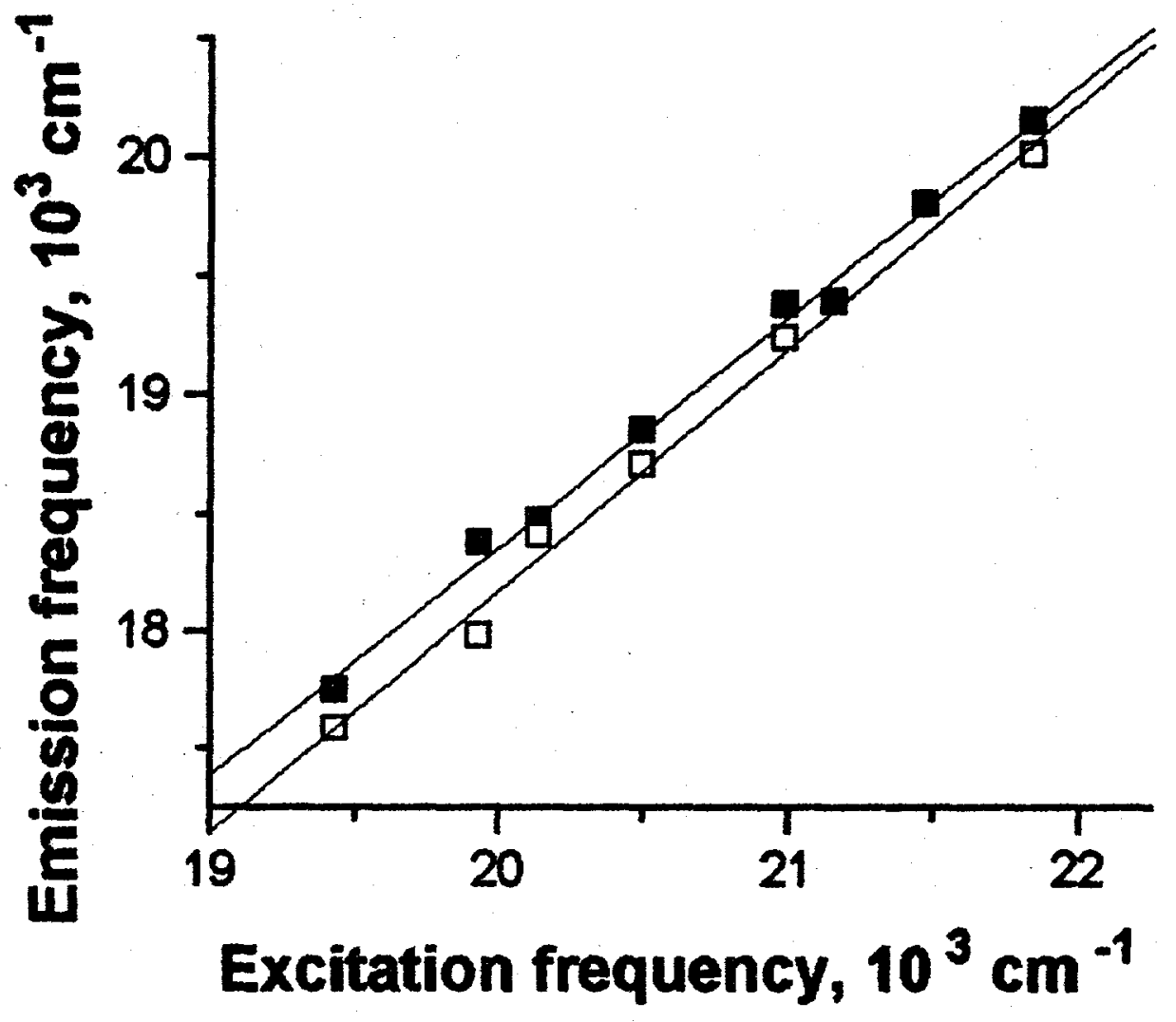

Fig.3(2.6). The dependence of the emission frequency of the main vibronic peak on the excitation frequency at room temperature (full squares) and at $10 \mathrm{~K}$ (open squares). The best fit straight lines have the slopes $1.02 \pm 0.05$ for the low temperature and $0.97 \pm 0.03$ for the high temperature. 
where $F_{0}$ and $A$ are constants and $E$ is the barrier height between two valleys. The approximation of our data by (1) gives $E=140 \pm 35 \mathrm{~cm}^{-1}$. The fitting curve is shown in Fig. 2(2.6). It was found for the p-terphenyl molecule that $E=190 \mathrm{~cm}^{-1}$ (at $300 \mathrm{~K}$ ) and $240 \mathrm{~cm}^{-1}$ (at $200 \mathrm{~K}$ ) [18]. Thus, our value obtained for PPP is of the same order of magnitude as that of $p$-terphenyl.

The observation of this unusial temperature dependence of the luminescence intensity can have some practical application. Probably it would be useful to heat slightly a sample to obtain higher photoluminescence and possibly electroluminescence intensity of PPP film.

The computer approximation of the luminescence spectrum obtained upon laser excitation at $21839 \mathrm{~cm}^{-1}$ at $50 \mathrm{~K}$ by six Gaussians in the $16600-20700-\mathrm{cm}^{-1}$ region gives the following transition frequencies $20380,19850,19150,18470,17780$ and $17070 \mathrm{~cm}^{-1}$. If the laser frequency is in resonance with the pure electronic transition (in the absence of energy relaxation) these transitions correspond to vibronic frequencies $\omega_{1}=1460, \omega_{2}=$ $1990, \omega_{3}=2690, \omega_{4}=3360, \omega_{5}=4060$, and $\omega_{6}=4770 \mathrm{~cm}^{-1}$. The first frequency $\omega_{1}$, corresponds to the ill-resolved shoulder of the intense peak at $\omega_{2}$.. The second frequency $\omega_{2}$ is close to that observed in the IR spectra of the initial PPP powder $\left(1930 \mathrm{~cm}^{-1}\right.$ in Fig.2 (2.4) of [1]) and of the PPP film $\left(1950 \mathrm{~cm}^{-1}\right)$ [14]. The third frequency $\omega_{3} \approx 2 \omega_{1}$. If the third peak is indeed an overtone of the first one, we can obtain the frequency $\omega_{1}$ more precisely because the third is better resolved. Therefore, $\omega_{1}=\omega_{3} / 2=1345 \mathrm{~cm}^{-1}$. This frequency is very close to the intense $\mathbb{R}$ line at $1375 \mathrm{~cm}^{-1}$ (see Fig. 3(2.4) in [1]) of the PPP film, which was assigned to the C-C skeletal vibration. The frequency $\omega_{4}$ is close to the $3030 \mathrm{~cm}^{-1}$ IR line assigned to the $\mathrm{C}-\mathrm{H}$ stretch vibration [14]. Frequencies $\omega_{5}$ and $\omega_{6}$ are possibly some overtones or composite vibrations $\left(\omega_{5} \approx 2 \omega_{2}\right)$.

The computer simulation of the two first peaks with fixed transition frequencies : $\omega_{1}=1375 \mathrm{~cm}^{-1}$ and $\omega_{2}=1930 \mathrm{~cm}^{-1}$ obtained from IR-spectra showed that the first peak (observed as a shoulder) broadens with temperature (Fig. 4(2.6)), whereas the spectral width of the second one is almost constant over the entire temperature range from 6 to $300 \mathrm{~K}$. The reasons of such a behavior are now unclear. One can suppose that the first vibronic transition $\left(\omega_{1}\right)$ is coupled with phonons having smaller dispersion of frequencies than that of phonons coupled with the second transition $\left(\omega_{2}\right)$.

The dependence of $v_{\mathrm{cm}}$ corresponding to the second vibronic transition $\left(\omega_{2}\right)$ on $v_{\mathrm{ex}}$ at $10 \mathrm{~K}$ is shown in Fig. 3(2.6). The similar dependence for room temperature obtained in [1] is also preseted in this figure. Some decrease in the emission frequency observed at 10 $K$ results from the low-temperature splitting of the peak discussed above. 


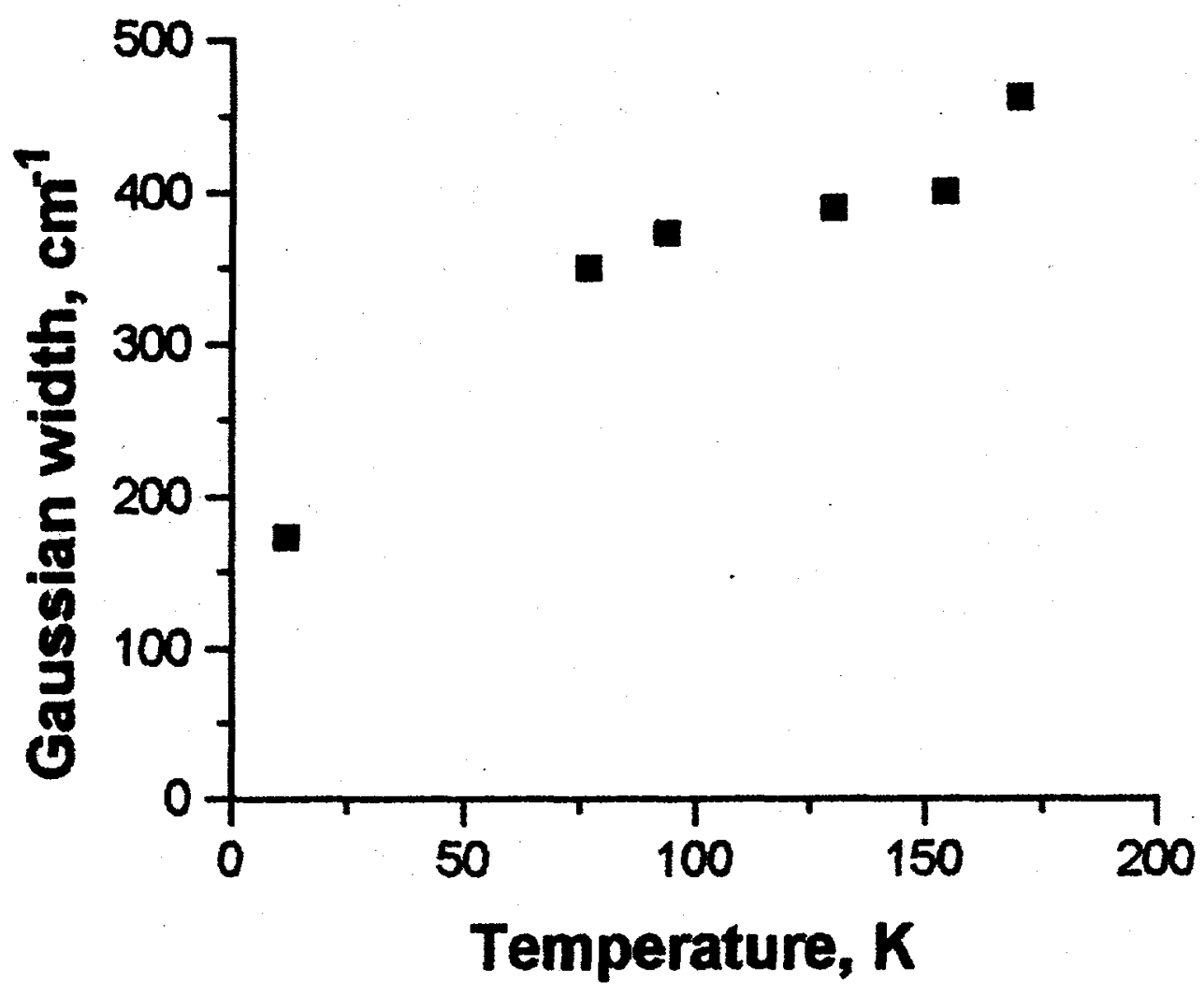

Fig 4(2.6) Temperature dependence of the Gaussian width of the vibronic peak; observed as a shoulder in Fig. 2 (peak 1). 


\section{References}

1. Report no. 1 (1995-1), March 25, 1995.

2.D.V.O'Conner and D.Phillips, Time-Correlated Single Photon Counting, Academic Press, 1984.

3.M. Komakine, T. Namikawa, and Y.Yamazaki Makromol Chem, Rapid Commun. 7 (1986) 139.

4.E.W.Knapp, Chem. Phys. 85 (1984) 73.

5.G.Leising, O.Leitner, F.Aldrian, and H.Kanlert, Synthetic Metals 17 (1987) 635.

6.K.Miyashita and M.Kaneko, Macromol Rapid. Commun. 15 (1994) 511.

7.A.Siemiarczuk, B.D.Wagnen, and W.R.Ware, J.Chem.Phys. 94 (1990) 1661.

8.V.M.Agranovich and M.D.Galanim, Electronic excitation energy transfer in condensed matter. North_Holland, Amsterdam, 1982.

9.M.Stavala, D.L.Dexter and R.S.Knox, Phys. Rev. B. 31 (1985) 2277.

10.Bkrabel, C.H.Lee, D.Mc.Branch, D.Moses, N.S.Sariciftyci, and A.J.Heeger, Chem

Phys. Lett. 213 (1993) 389.

11. A.Aeiyach, P.C.Lacaze, J.Chem.Phys, Phys.-Chim Biol. 86, 143 (1989).

12. Z.Mo, K.B.Lee, Y.B.Moon, M.Kobayashi, A.J.Heeger, F.Wudl, Macromolecules $18,1972(1985$.

13. P.Kovacic, M.B.Feldman, J.P.Kovacic, J.B.J.Lando, Appl.Plym. Sci. 12, 1735 (1968).

14. L.W.Schacklette, R.R.Chance, D.M.Ivory, G.G.Miller, R.H.Baughmann, Synth.Met.1, 307 (1979).

15. F.Teraoka, T.Takahashi, J.Macromol.Sci., Phys. 18, 73 (1980).

16. M.Komakine, T.Namikawa, Y.Yamazaki, Makromol.Chem, Rapid Commun. 7, 139-142 (1986).

17. T.Yamamoto, A.Morita, Y.Miyazaki, T.Maruyama, H.Wakayama, Z.Zhou, Y.Nakamura, T.Kanbara, Macromolecules 25, 1214-1223 (1992).

18. J.L.Baudour, H.Cailleau, and W.B.Yelon, Acta Cryst. B33, 1773 (1977).

19. N.I.Wakayama, J.Lumin. 27, 299 (1982).

20. K.Uchida, S.Sato, and Y.Takahashi, J.Lumin. 48-49, 377 (1991). 


\section{STUDY OF J-AGGREGATES}

\subsection{Introduction}

It has been discovered long ago that cyanine dyes can form specific aggregates with very narrow absorption band shifted to the red relative to that of monomer [1,2].

Polarization data in the streaming solutions suggested that the linear-extended structure of J-aggregates have [3]. Physical length of them is not well established and is estimated to be from $10^{1}$ to $10^{5}$ of molecules [4]. These systems are quite well organized and as in the case of molecular crystals, their excited states can be described by Frenkel excitons. Exciton motion in J-aggregates is considered to be restricted by one dimension and finite chain length. However, it turns out that the model of the ideal rigid linear chain can not describe all optical properties of such systems. J-aggregates are usually embedded in amorphous media, that implies the greater structural disorder as compared to molecular crystals. This disorder can be described by the imhomogeneous distribution of energies of sites (diagonal disorder) and by the distribution of resonance coupling constants between sites (non-diagonal disorder) [5,6 and references therein]. Moreover, in reality some defects can break the whole chain into the relatively decoupled segments. The segments are usually assumed to be defect free linear chains of finite lengths. The disorder results in the inhomogeneous line broadening, although the stationary states are still delocalized over particular segment lengths. In addition, the real chain is not rigid and its vibrations affect the exciton dynamics through exciton-phonon coupling. Thus, J-aggregates in solutions are highly anysotropic (quasi-one-dimensional) restricted systems with static and dynamical disorder.

However, the degree of topological anisotropy and therefore the applicability of the one-dimensional chain model for J-aggregates are not established experimentally so far. Even indirect indications of fractal dimensionality are reported in the literature [7]. The exciton-phonon interaction in lattices of different dimensionality results in qualitatively different temperature behavior of optical spectra [8]. In particular, very interesting phenomenon of the exciton self-trapping by lattice phonons can reveal itself variously, depending crucially on the system dimensionality and size restrictions [9-14]. It has been found theoretically for 3D system that if the parameter of exciton-phonon coupling exceeds some critical value, the stable self-trapped state (ST) appears below the bottom of the band of free excitonic states (F) $[9,10]$. In this case two energy minima are separated by a potential barrier. On the other hand, as has been shown by Rashba [9] in infinite strictly 1D chain the self-trapping occurs for any non-vanishing exciton-phonon coupling and the barrier between ST and F states is absent. In this case the stable state is 
the ST state at any coupling constant. If however, the system is not strictly ID, possessing nevertheless a high anisotropy (quasi-one-dimensional), the potential barrier reappears $[15,16]$.

There are only few examples of experimental observation of self-trapping in 3-D organic crystals with molecules capable to form excimers ([17] and references therein). This limitation results from a lack of long-range interaction of Frenkel excitons with optical phonons and from a requirement of quite strong interaction with acoustical phonons [11]. Note, however, that for quasi-one dimensional systems, these limitations are lifted [11]. Nevertheless, experimental observations of ST states in such systems are also few in number. To mention only the experiments on tetracyanoplatinates [16], halogen-bridged mixed-valence metal complexes [18] and organic p-conjugated polymers $[19,20]$.

Self-trapping in J-aggregates has not been established directly, but some indirect indications of this process are present in the literature [21,22]. In [21] the very wide and intense red tail in the fluorescence spectrum has been observed at liquid helium temperatures, transforming to the separate wide red-shifted peak at $77 \mathrm{~K}$ [21b]. Authors attributed these spectral features to self-trapping of excitons assisted by excimer formation. The mathematical analysis of fluorescence decay kinetics in the region of red peak provides an evidence against the trapping by low-energy monomer traps [21b]. Also, the non-exponentiality of decay and the red shifting of time-resolved fluorescence spectrum suggest the emission from unrelaxed ST state. In another communication [22a] the appearance of the red tail in the fluorescence spectrum and the broadening of the spectrum during hundreds of picoseconds was attributed to the self-trapping mechanism as one of possibilities.

Our work is devoted to the experimental investigation of.exciton relaxation in molecular J-aggregates of thiacarbocyanine dye and the influence of electron-phonon coupling on their optical spectra. It has been found experimentally that the fluorescence spectrum is much more sensitive to the temperature change in the range $5-140 \mathrm{~K}$ compared to the absorption spectrum. Thus, we paid particular attention to the temperature dependencies of the shape, width and red tail of the fluorescence spectrum. In intermediate temperature region $40-120 \mathrm{~K}$ an additional "red" peak coexists with the main "blue" peak. At $\mathrm{T}<30 \mathrm{~K}$ and $\mathrm{T}>120 \mathrm{~K}$ only the blue peak is observed.

Fhorescence polarization experiments have been performed to obtain additional information on molecular structure corresponding to these two peaks. 
Our observations indicate the quasi-one-dimensional character of exciton motion in $\mathrm{J}$-aggregates. We recognize two exciton relaxation processes both resulting in some exciton localization.

Persistent spectral hole burning experiments show the significance of processes with one-phonon emission even at very low temperatures. This leads us to the conclusion that the fluorescence spectrum of the "blue" peak can be formed by clothed (or dressed) excitons which can be associated with the first steps of trapping process. Detailed analysis of the "blue" peak based on fluorescence spectroscopy at low temperatures suggests the barrierless self-trapping process is essential in formation of this peak.

The appearance of additional maximum in fluorescence spectrum at intermediate temperatures is discussed in terms of temperature-induced chain photo-rearrangement involving tunneling through potential barrier at low temperatures $(T<30 \mathrm{~K})$.

\subsection{Experimental}

The 3,3',9-triethyl-5,5'-dichlorothiacarbocyanine iodide (TDC) dye was synthesized at INFO-PHOTO LTD., Moscow and used without further purification.

For preparation of J-aggregates the TDC was dissolved in a 3:2 volume water / ethylene glycol (WEG) mixture upon heating up to $70{ }^{\circ} \mathrm{C}$. The solutions with dye concentration of $\sim 3 \times 10^{-3} \mathrm{M}$ were cooled to room temperature, put into a dismountable glass cell 200 or $400 \mu \mathrm{m}$ in thickness, then cooled in an Oxford Instruments CF-204 helium cryostat to $250 \mathrm{~K}$ and kept at this temperature for 10-30 min. At this stage a narrow J-band appears at $618 \mathrm{~nm}$ in absorption spectrum of the dye. Then the samples were placed into liquid nitrogen where they were frozen for about $30 \mathrm{~s}$, again placed into a cryostat at $80 \mathrm{~K}$ and finally cooled down to liquid helium temperature. Some experiments were accomplished at $77 \mathrm{~K}$ in liquid nitrogen cryostat.

Fluorescence excitation and hole burning were performed with an argon laserpumped cw Coherent CR-699-21 ring dye laser operated with Rhodamine 6G in multimode regime with a linewidth of about $0.1 \mathrm{~cm}^{-1}$. The transmission spectra were recorded using a stabilized filament lamp and a double spectrometer. The hole spectra were detected with a resolution $0.6-1 \mathrm{~cm}^{-1}$. A procedure of obtaining of the true hole width is described in Section 5. Picosecond fluorescence lifetime measurements were performed using the Antares 76-dye 702-system (Coherent). The fluorescence signal was detected by method of up-conversion. The system and method were described in the Report Part 1, sect.2.1. 


\subsection{Results and Discussion}

\subsubsection{General View of Spectra of Samples with J-Aggregates of TDC}

An increase of concentration of the TDC in water and WEG solutions results in the intense broad absorption at $520 \mathrm{~nm}$ (usually assigned to the H-band and dimers) and two narrow bands at 640 and $618 \mathrm{~nm}$. The latter two narrow bands are red-shifted related to the monomer absorption maximum $(554 \mathrm{~nm})$ and correspond to different types of $\mathrm{J}$ aggregates, Fig.1(3.3). The intensity of the short-wave $(618 \mathrm{~nm})$ band increases gradually with the cuvette thickness, whereas that of the long-wave $(640 \mathrm{~nm})$ does not depend on this parameter. This can imply that the long-wave band belongs to J-aggregates formed at the cell walls, whereas the short-wave band is related to J-aggregates formed in the bulk of solution. In the $400 \mu \mathrm{m}$ - thick cell the short-wave J-aggregates dominate. The $640 \mathrm{~nm}$ band almost disappears upon freezing even in thin cells. In this work we restrict ourselves to the study of the short-wave band, (which can have, however, its own structure, see below). The absorption and fluorescence short-wave J-bands of TDC in WEG solution at $6 \mathrm{~K}$ are presented in Fig.2(3.3). In frozen solution the absorption maximum is shifted to the blue by $7 \mathrm{~nm}$ and occurs at $611 \mathrm{~nm}\left(16360 \mathrm{~cm}^{-1}\right)$. One can see, that the fluorescence band at $5 \mathrm{~K}$ is narrower and shifted to the red (by ca. $100 \mathrm{~cm}^{-1}$ ) with respect to the absorption band. Moreover, the fluorescence band is asymmetric with abrupt edge on the high-energy side and the smooth fall in the low-energy side. The absorption spectrum has a more symmetric shape.

It has been found that the fluorescence spectrum is much more sensitive to the temperature change in the $5-140 \mathrm{~K}$ range compared to the absorption spectrum. At the intermediate temperatures the short-wave fluorescence J-band reveals additional structure (Fig.3(3.3)). However, in absorption this additional red peak is absent, see Fig.4(3.3).

It has been established earlier that J-aggregates of TDC cloride can exist in three different forms in aqueous solutions at room temperature [23]. Their absorption maxima have been found at $642 \mathrm{~nm}\left(J_{1}\right), 623 \mathrm{~nm}\left(J_{2}\right)$ and $619 \mathrm{~nm}\left(J_{3}\right)$. The $J_{1}$-aggregate was shown to reveal no high stability and to be readily transformed into the latter two forms. The $J_{3}$-aggregate was prepared by heating of the TDC solution up to $50^{\circ} \mathrm{C}$ or by stirring. It has also been discovered that $\mathbf{J}_{\mathbf{2}}$ and $\mathbf{J}_{\mathbf{3}}$ aggregates can coexist in some cases [23]. As was mentioned above we do not consider the $\mathrm{J}_{1}$-band there because it disappears upon freezing. The assignment of our red and blue fluorescence peaks, coexisting in frozen solution to the $J_{2}$ and $J_{3}$-aggregates of Hada respectively is based on their spectral positions (taking into account the blue shift after freezing) and separation. Thus, we shall use below these notations for our red and blue peaks. However, in contrast to results of 


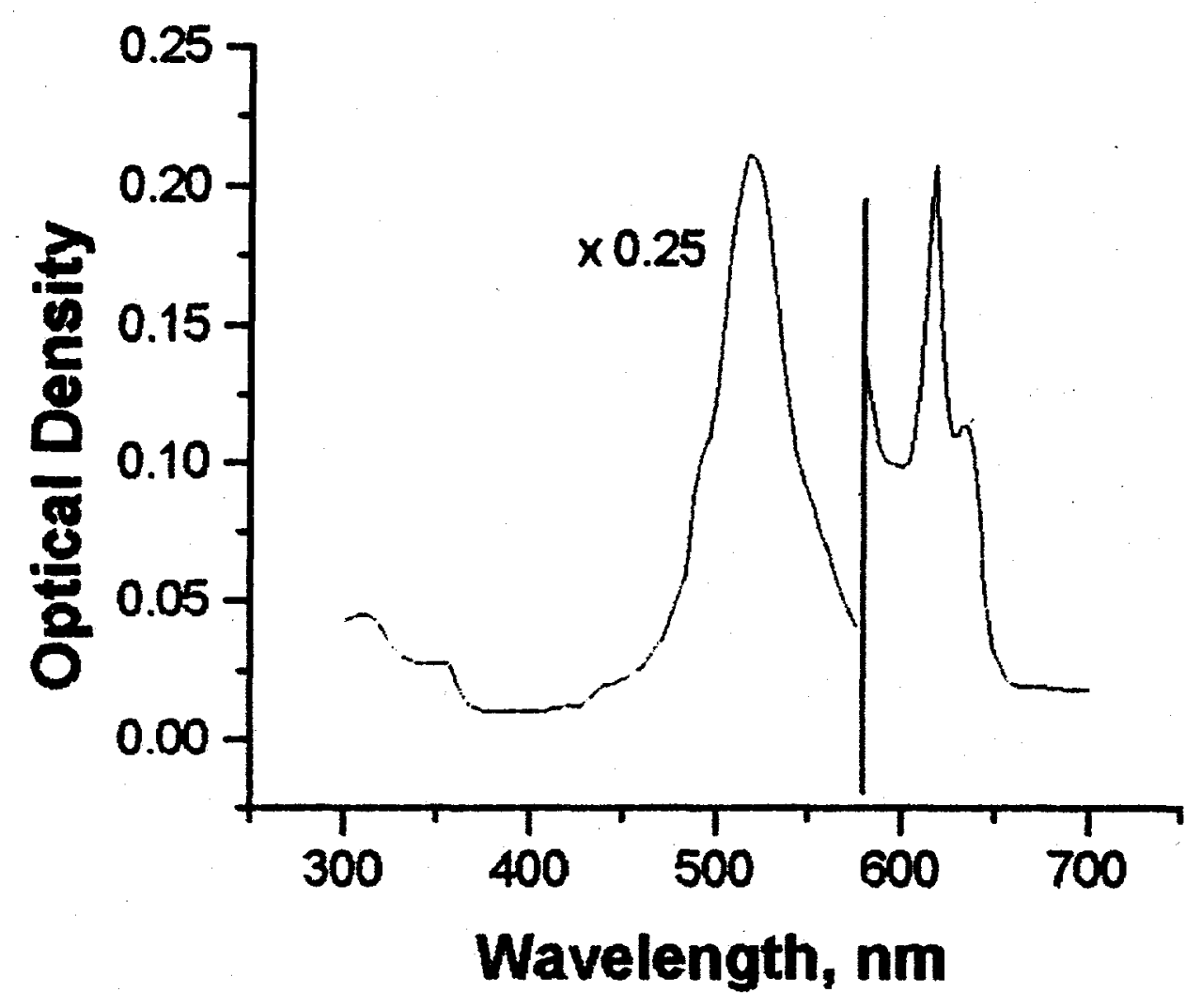

Fig.1(3.3). Absorption spectrum of $10^{-3} \mathrm{M}$ water solution of TDC at room temperature. The short-wave portion is suppressed by a facor of 0.25 . 


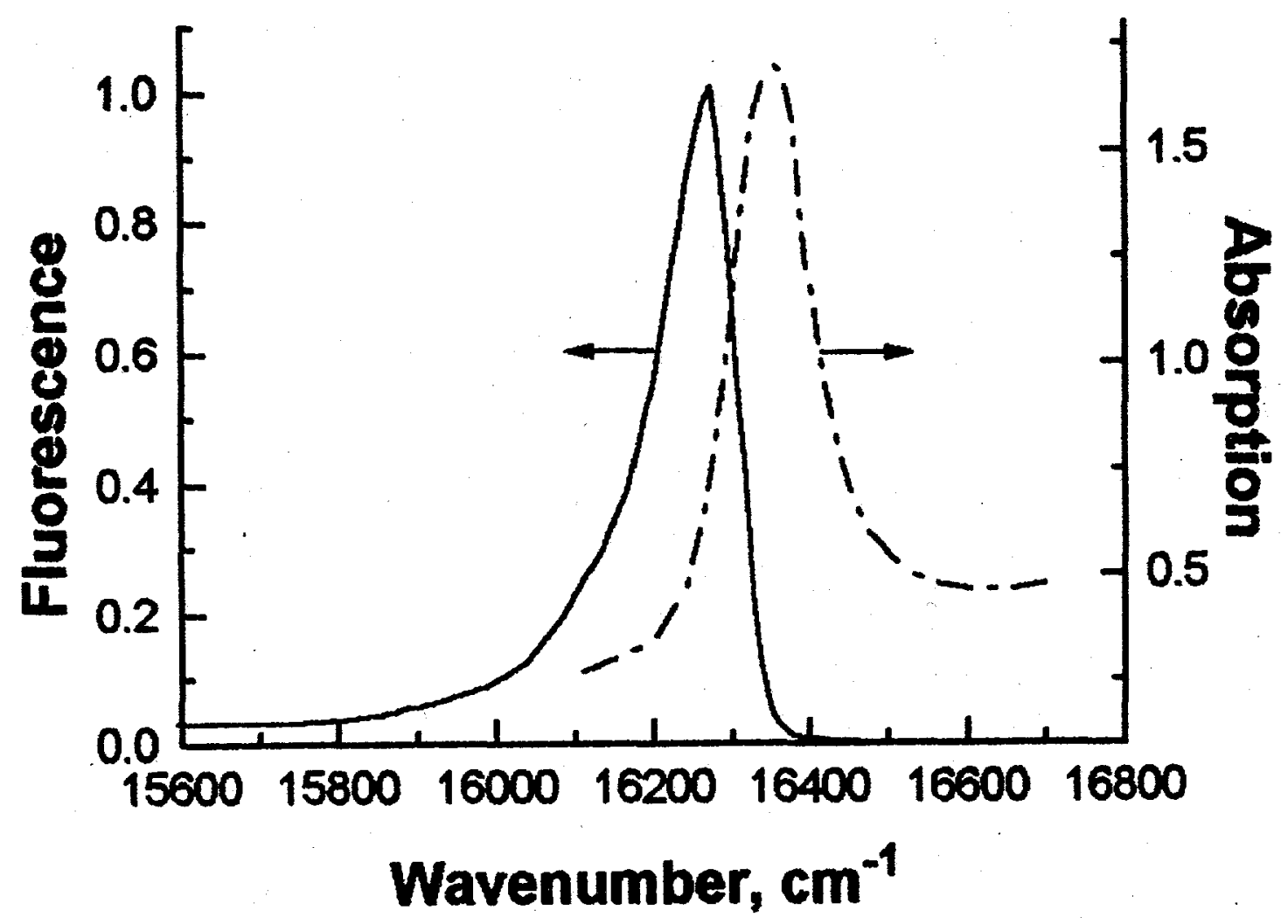

Fig.2(3.3). The absorption and fluorescence J-bands in frozen matrix solution at $6 \mathrm{~K}$. 


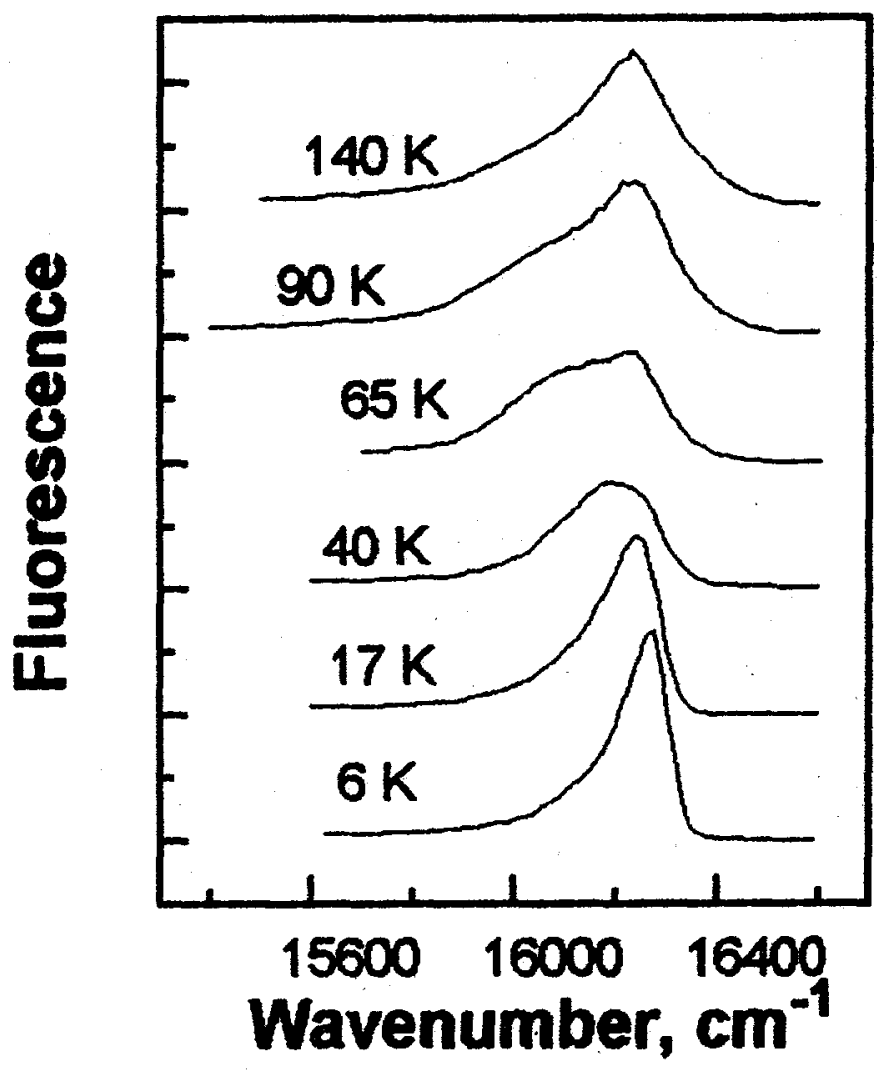

Fig.3(3.3). Fluorescence spectra at different temperatures shifted along the ordinate axis for clarity. 


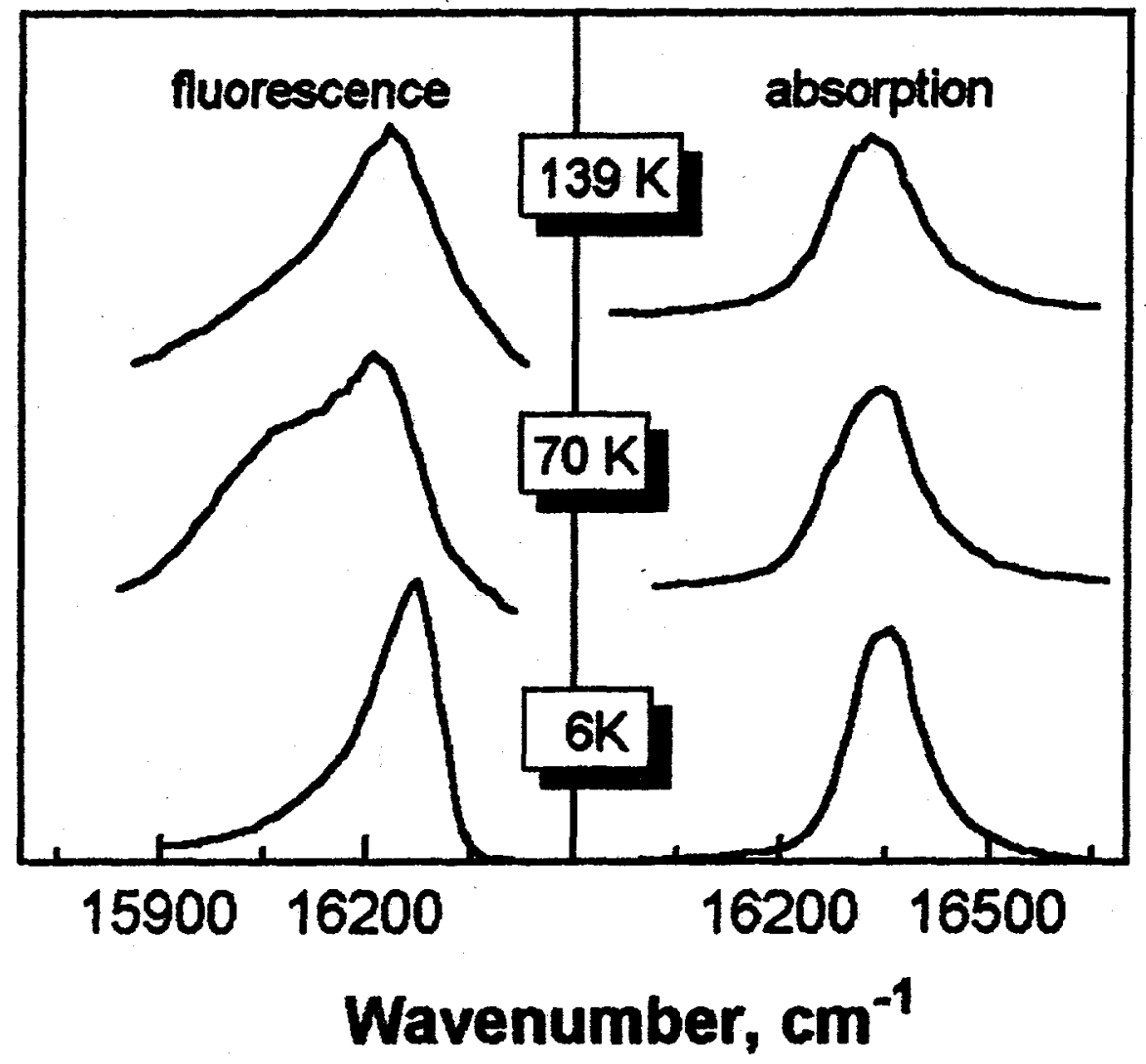

Fig.4(3.3). Representative spectra of fluorescence and absorption at three different temperatures. 
Hada al, we observed in fresh samples the coexistence of $J_{2}$ and $J_{3}$-bands only in fluorescence spectrum, converting from one to another as temperature changes. The absorption spectrum usually does not exhibit $J_{2}$ peak at any temperature (see Fig.4(3.3)). Only in rare cases (for aged samples) we obtained the absorption spectrum with an appreciable red shoulder. In these samples the red peak in the fluorescence spectrum dominates over the blue one and their relative intensities are independent of temperature.

\subsubsection{Fluorescence Polarization}

The fluorescence anisotropy spectrum of our sample at $77 \mathrm{~K}$ is shown in Fig.5(3.3). The steady state fluorescence anisotropy value $A=\left(I_{11}-I_{\perp}\right) /\left(I_{11}+2 I_{\perp}\right),\left(I_{11}, I_{\perp}\right.$ are the fluorescence intensities polarized parallel and perpendicularly to excitation polarization) in a rigid matrix reflects the angle between transition dipoles of excitation and emission. One can see from Fig.5(3.3) that the $A$ for bhe peak is negative and high in absolute value if the fluorescence is excited beyond the J-band. This fact agrees well with the assumption that the angle between the absorption dipole (H-band) and the emission dipole (J-band) is close to $90^{\circ}$ [23b]. Rather more intriguing fact is a small variation (by less than 30 percents) of anisotropy in passing to the red peak and the reproduction of the fluorescence spectrum structure in the anisotropy spectrum. This persistence of anisotropy value from the blue to the red peak suggests the absence of energy transfer between randomly oriented units. Indeed, if the latter occurs, the fluorescence would be virtually almost depolarized after a single transfer act [24]. Therefore, if the energy transfer process really populates the red states of our system the angle between the dipole moments of segments responsible for the blue and red peaks in fluorescence should be small enough. In other words, the segments can not belong to different chains randomly oriented with respect to each other. Moreover, the fluorescence anisotropy vahues, recorded at maxima of each peak vary in parallel as a function of excitation wavelengths and become positive upon resonance excitation in J-band, see Fig.6(3.3). These results show that emission dipole moments of both peaks are highly correlated in orientation.

\subsubsection{Temperature Dependent $J_{3} \rightarrow J_{2}$ Photoconversion}

A rather interesting result is the temperature dependent phototransformation of the $J_{3}$ form to the $J_{2}$ form. As the temperature increases from 5 to $40 \mathrm{~K}$ the fluorescence maximum shifts to the red and the band broadens considerably, Fig.3(3.3). At 30 - $40 \mathrm{~K}$ an additional red shifted shoulder (or resolved peak in other samples) appears against the background of the smooth red wing. The relative intensity of this red $\mathrm{J}_{2}$-peak reaches a 


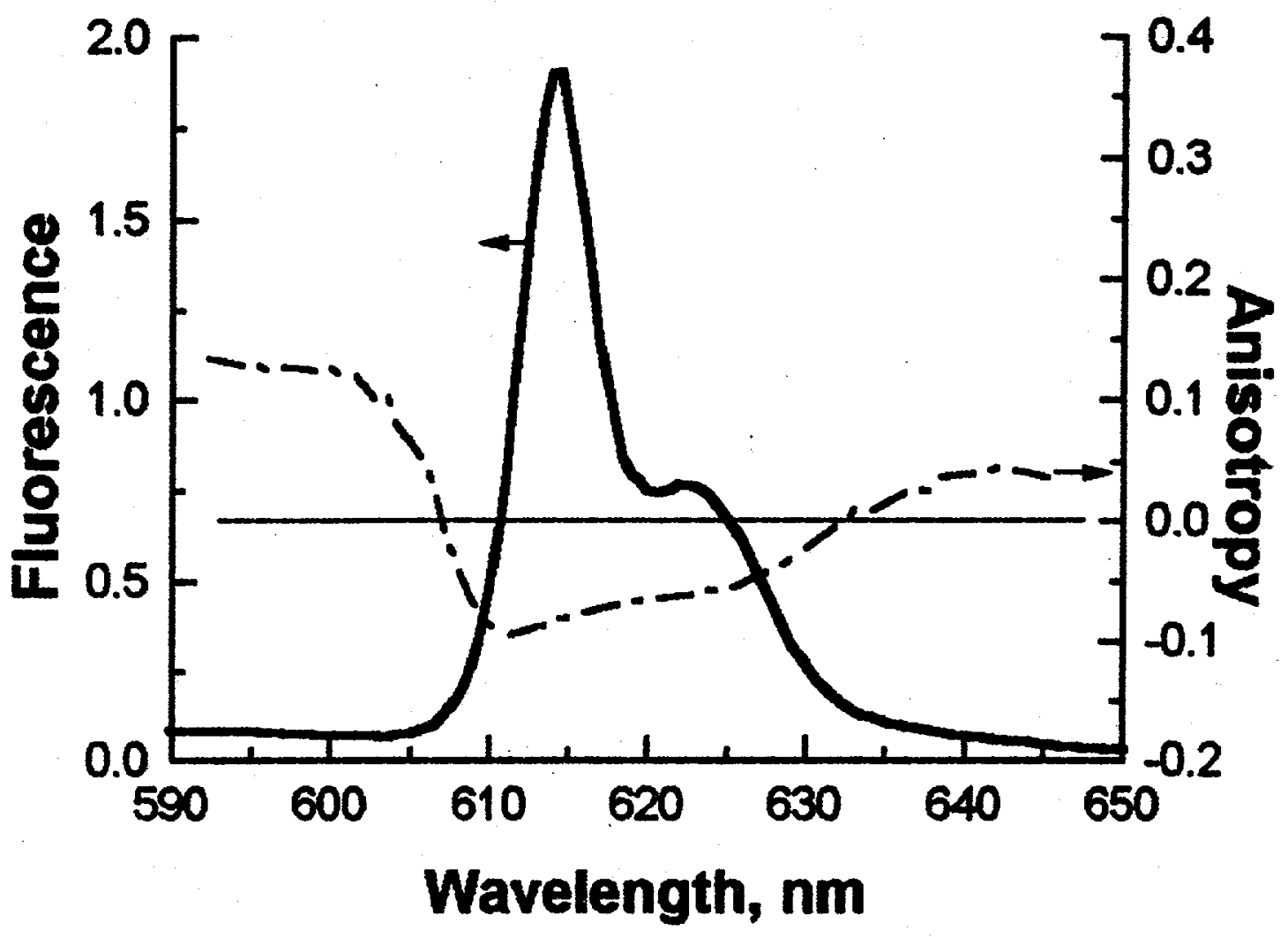

Fig.5(3.3). Anisotropy of fluorescence as a function of emission wavelength at $77 \mathrm{~K}$ upon off-resonant excitation at $575 \mathrm{~nm}$. The fluorescence spectrum is also shown for comparison. 


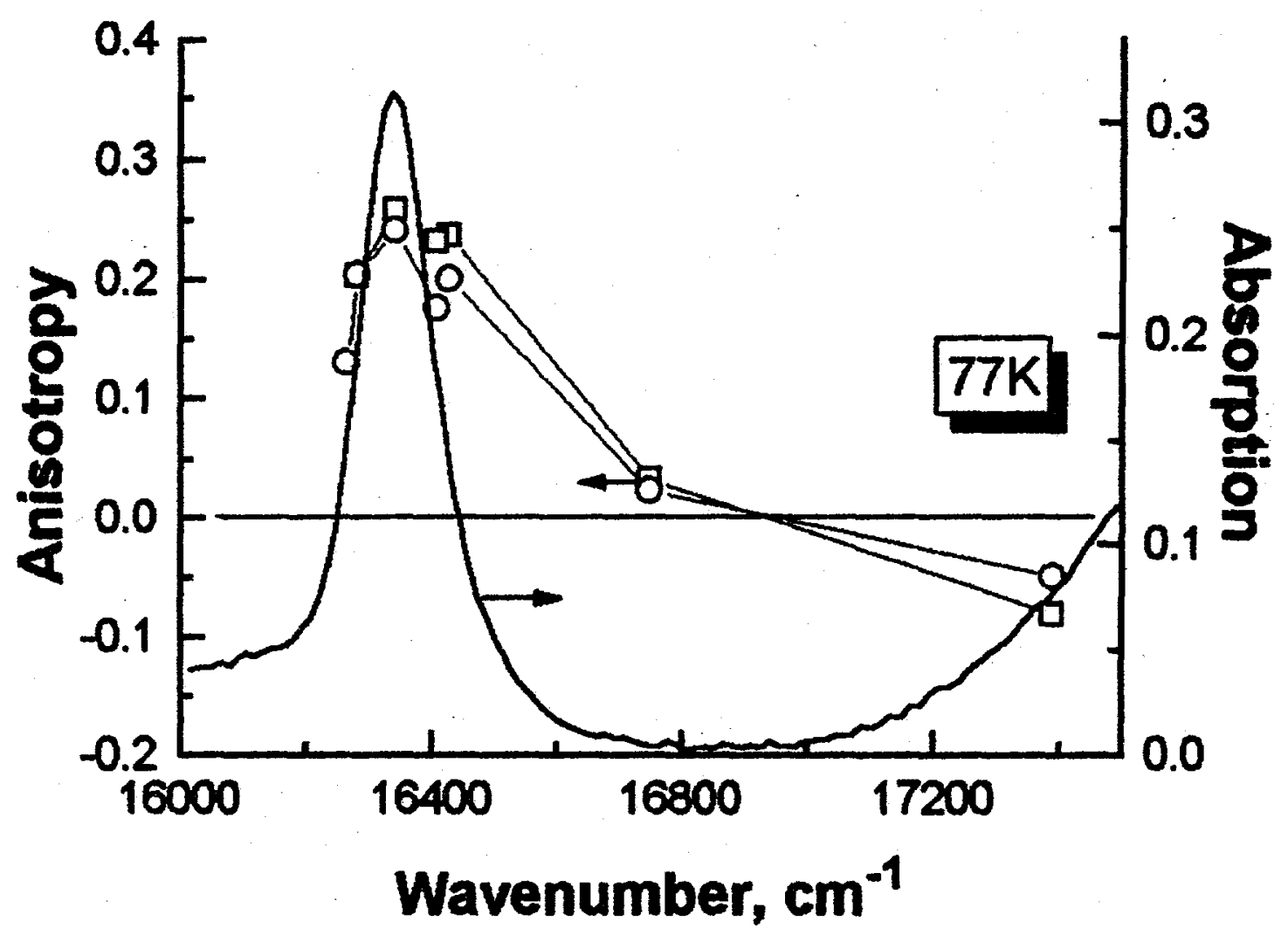

Fig.6(3.3). Anysotropy of fluorescence as measured at the maximum of the $J_{2}$-peak (circles) and at the maximum of the $\mathrm{J}_{3}$-peak (squares) at $77 \mathrm{~K}$. The absorption peak is also presented. 
maximum at $40-60 \mathrm{~K}$ and decreases smoothly with a further increase in temperature (see Fig.3(3.3)). One can see that at $100-140 \mathrm{~K}$ the red shoulder disappears. At these temperatures the fluorescence spectrum has again the red wing without any inflections and maxima. It should be noted that the disappearance of the red band at elevated temperatures is not due to the simultaneous broadening of both peaks. The spectrum at $139 \mathrm{~K}$ is even narrower that that at $70 \mathrm{~K}$.

Note that the relative intensity of the $\mathrm{J}_{2}$-band depends on the sample preparation procedure but has a similar temperature dependence for different samples (Fig.7(3.3)). The $J_{2}$-peak (if resolved) has a width 1.5 - 2 times greater than that of the $J_{3}$ peak.

As was mentioned above in the aged samples $J_{2}$ and $J_{3}$ bands can sometimes coexist in absorption spectrum as well, but they do not convert to each other either in absorption or in fluorescence spectra as temperature changes. These facts suggest very interesting result that the $\mathbf{J}_{2}$-form can be prepared either beforehand by special procedure [23a] or only by virtue of transformation of excited $J_{3}$-aggregate. In the latter case the $J_{2^{-}}$ band is observed only in the fluorescence spectrum.

Another evidence of conversion of excited $J_{3}$ to $J_{2}$-aggregate is the dependence of their relative intensity on the excitation wavelength, shown in Fig.8(3.3). .The relative intensity of two peaks depends only slightly on the excitation frequency, while their individual absorption spectra are qualitatively different in this frequency range [23a]. The extinction coefficient for $J_{3}$-aggregate rises from $600 \mathrm{~nm}$ to the blue, whereas that of $J_{2}$ aggregates falls down [23a]. Thus, one would expect a decrease in the relative red $\left(J_{2}\right)$ peak intensity with increasing excitation frequency. However, the situation is reversed (see Fig.8(3.3)).

The fact that $J_{3}$ and $J_{2}$ peaks coexist in the fluorescence spectrum, whereas in the absorption spectrum only the $J_{3}$ peak is observed can be explained either by the energy transfer from $J_{3}$ to $J_{2}$ or by structural rearrangement upon excitation. There are, however, several experimental findings which are difficult to interpret within the framework of the energy transfer model. The non-monotonic temperature dependence of the fluorescence spectrum is the strongest indication against simple energy transfer mechanism of population of states responsible for the red peak. Although, in some doped molecular crystals the energy transfer rate is found to be temperature dependent $[25,26]$, the relative luminescence intensity of donor and acceptor changes monotonically [25].

The non-monotonic qualitative changes in the fluorescence spectrum with temperature suggest that the process analogous to self-trapping of exciton on lattice deformation takes place. Indeed, Sumi and Toyozawa [27] calculated luminescence spectrum and shown that an additional red peak in the luminescence spectrum, 


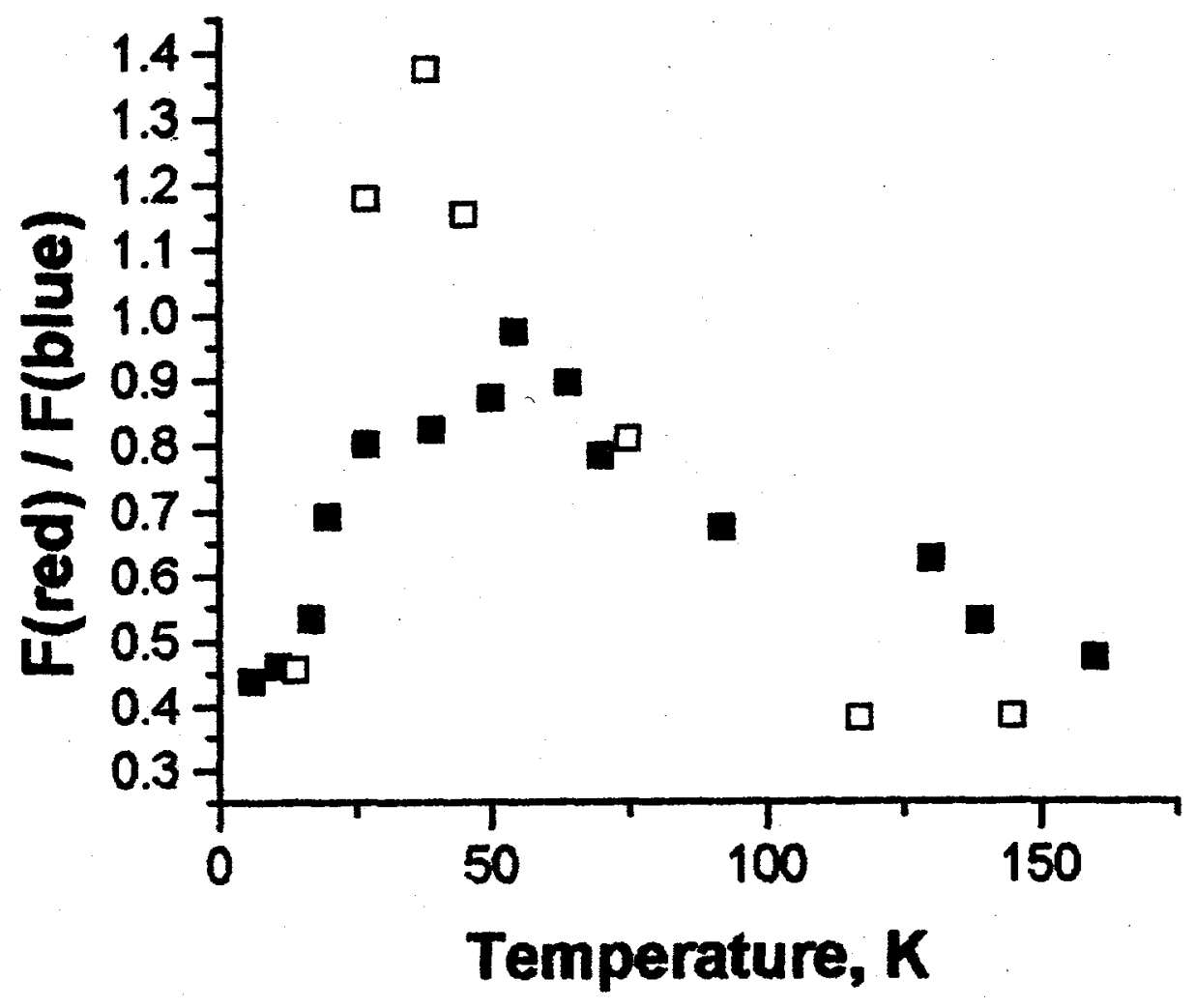

Fig.7(3.3). Temperature dependence of the intensities ratio in the $J_{2}$ (as measured at $16000 \mathrm{~cm}^{-1}$ ) and $J_{3}$ (as measured at the maximum of the peak) peaks. Full squares are for fresh sample and open squares are for two weaks aged sample. 


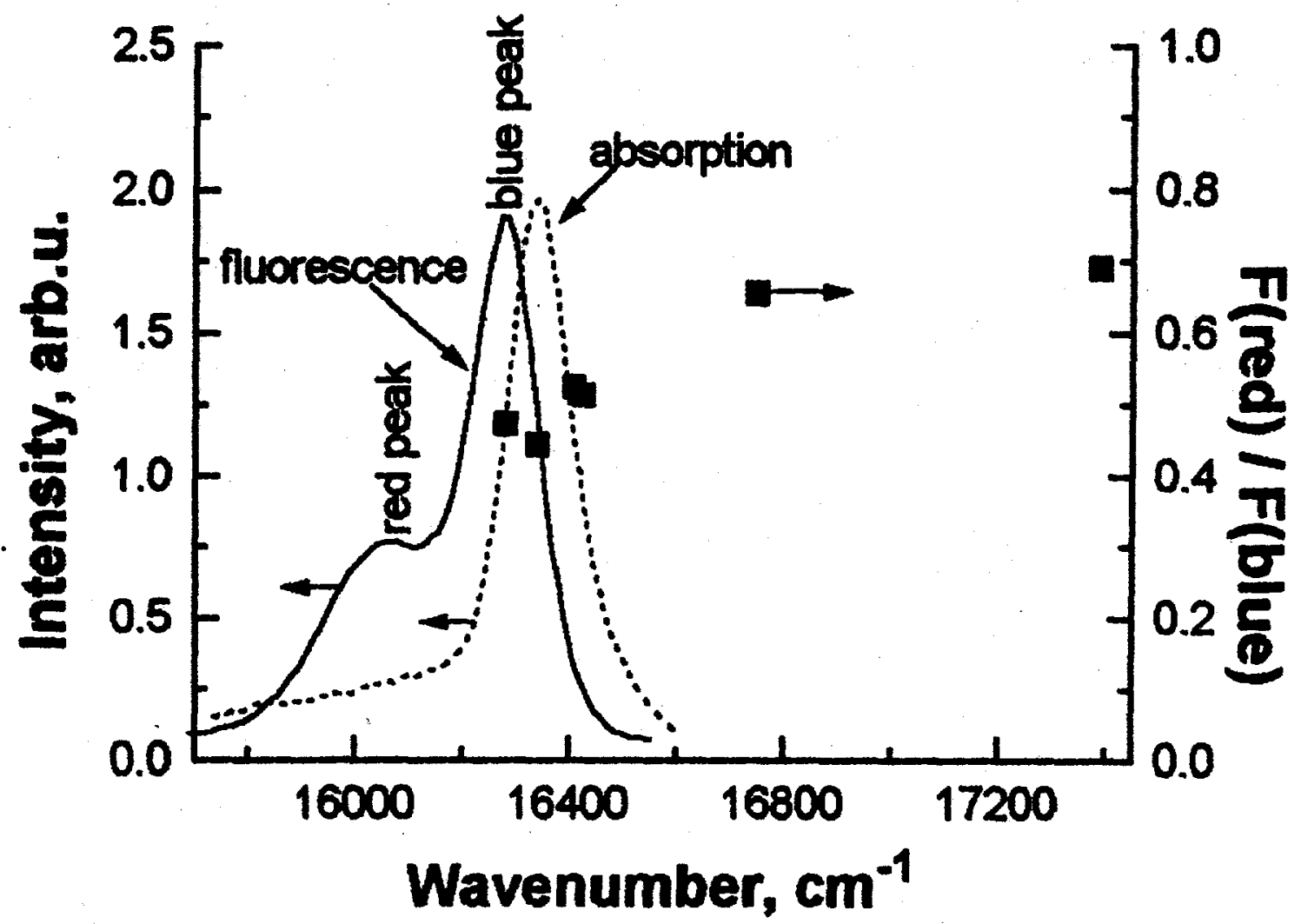

Fig.8(3.3). The dependence of the intensities ratio in the $J_{2}$ and $J_{3}$ peaks on excitation wavelength (full squares) at $77 \mathrm{~K}$. The absorption and flurorescence spectra at this temperature are also shown. 
corresponding to the deeper minimum in adiabatic potential can appear for suitable electron-phonon coupling strength. Furthermore, they predict this peak to disappear at elevated temperatures. This is due to the effect of giant oscillator strength of trapped states lying very close to the bottom of the free exciton band [28]. At sufficiently high temperatures these states are thermally populated. As for absorption, no additional red peak was predicted by the model [27] at any electron-phonon coupling strength. Therefore, our experiments are well described by the model of two energy minima in the excited state separated by the potential barrier (Fig.9(3.3)): The disappearance of the red peak at $\mathrm{T}>100 \mathrm{~K}$ is a strong evidence in favor of the trapping mechanism. The model of Sumi and Toyozawa does not take into account a finite lifetime of the excited state and thus does not show the absence of the red peak in luminescence spectrum at low temperatures. If, however, the free exciton lifetime is much smaller than the time of tunneling through potential barrier, the ST state could not be populated during excitation. The drastic rise of the red peak in fluorescence at around $30-40 \mathrm{~K}$ in our case (see Fig.7(3.3)) implies the overcoming of potential barrier of ca. $30 \mathrm{~cm}^{-1}$ height.

There is a close correspondence of our spectral results with kinetic measurements of Kamalov et al. in J-aggregates of another dye (BIC) [22]. The activation energy of the non-radiative channel of the electronic relaxation was measured [22b] to be of $30-40 \mathrm{~cm}^{-}$

1. The broadening of the fluorescence spectrum and the appearance of the red tail occur during $300-500$ ps at $4.5 \mathrm{~K}$ [22a], but these changes occur without any measurable delay at $\mathrm{T}>50 \mathrm{~K}$ [Kamalov, private communication]. On the other hand, the lifetime at liquid helium temperatures is only $100 \mathrm{ps}$ in BIC J-aggregates [22b].

Thus, the fact of self-preparation of $\mathrm{J}_{2}$ from the excited $\mathrm{J}_{3}$-form could be related in a most reasonable way to the process of self-trapping of exciton involving chain deformation or realignment by the electronic excitation. This is based upon the observed broadening of the $\mathrm{J}_{2}$-band and its behavior at elevated temperatures (see above). The two states $\left(J_{3}\right.$ and $\left.J_{2}\right)$ are separated by a potential barrier, that is in apparent contradiction with infinite perfect ID system. But this can be overcomed supposing some deviation from strict one-dimensionality of J-aggregate structure (e.g. [7]). In this case the potential barrier reappears. This has been shown theoretically and experimentally for quasi-onedimensional semiconductors $[15,16]$.

The molecular structure of two forms of aggregates under investigation $\left(\mathrm{J}_{2}\right.$ and $\left.\mathrm{J}_{3}\right)$ in solution is not established at present. In general, different physically distinct linear arrangements, revealing appreciable red-shifted J-bands have been discussed in literature [29-31]. The most frequently used are the staircase arrangement, the brickstone work arrangement and the $\mathrm{W}$-like arrangement. In the dipole-dipole approximation of the 


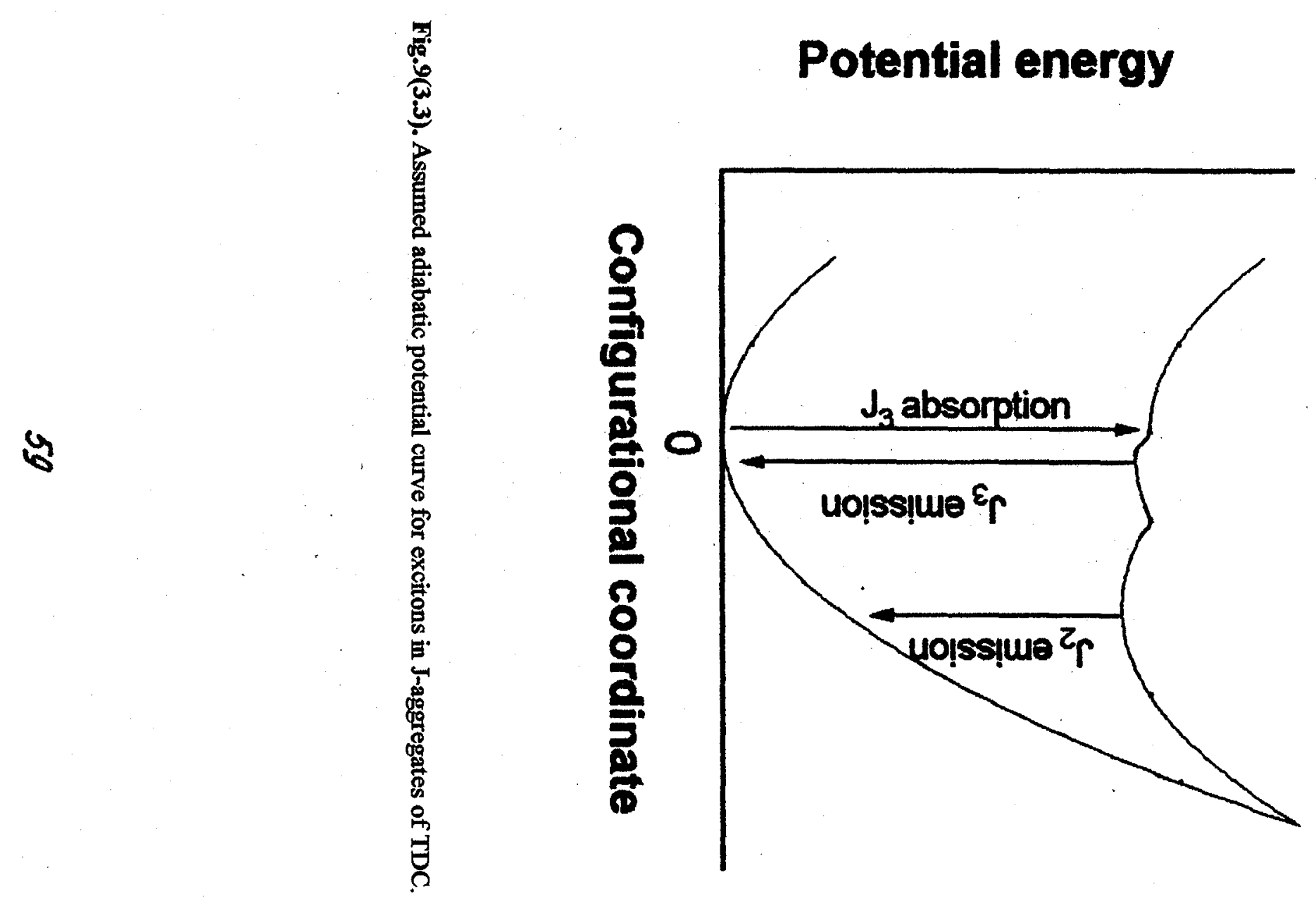


intermolecular transfer integral the staircase arrangement has only one allowed optical transition depending on the tilt angle of monomer long axes with respect to chain axes. For small angles $\left(\alpha<54.7^{\circ}\right)$ only a transition to the bottom of excitonic band (J-transition) is allowed, but for large angles $\left(\alpha>54.7^{\circ}\right)$, only a transition to the top of the band (Htransition) is allowed [30]. The TDC $\mathrm{J}_{3}$-aggregate in solution posses a strong $\mathrm{H}$ transition, whereas the J-transition is weaker (see [23] and Fig.1(3.3)). On the other hand, for the system revealing only $\mathrm{J}_{2}$-aggregate, the $\mathrm{H}$-transition is completely forbidden [23]. This could imply the staircase or W-like arrangements with large tilt angles ( $\alpha>54.7^{\circ}$ or $\alpha>45^{\circ}$, respectively) for the $\mathrm{J}_{3}$-form. The $\mathrm{J}_{2}$-form can have either the staircase and $\mathrm{W}$-like structures with small angles or the brickstone work arrangement. (Reich et al. [32] assigned the $620-623 \mathrm{~nm}$ band of J-aggregate of TDC on AgBr surface to the $30^{\circ}$-tilt staircase structure. This assignment was based on the hypothesis of epitaxial adsorption.) Simple calculations based on McRae and Kasha's model [30] show that the slight decrease in the tilt angle in the staircase structure e.g. from $60^{\circ}$ to $49^{\circ}$ gives rise to a drastic redistribution of transition intensities from $\mathrm{H}$-transition to the J-transition. Moreover, this rearrangement leads to the broadening of excitonic band by a factor of 1.1 and, consequently, the shift of the transition from $618 \mathrm{~nm}\left(J_{3}\right)$ to $623 \mathrm{~nm}\left(J_{2}\right)$. While the simple point dipole approximation is questionable for tight-binding aggregates [31] it can qualitatively account for the spectral changes during the process of $J_{3}-J_{2}$ rearrangement. Therefore, the self-trapping of more delocalized exciton in the $J_{3}$-form can be accomplished by structural rearrangement (e.g. tilt angle decreasing) giving rise to less delocalized exciton in the deformed lattice possessing the $\mathbf{J}_{2}$-band in fluorescence.

\subsubsection{Energy-Selective Spectroscopy}

Let us now concentrate on the optical properties of only $J_{3}$-band. As has been mentioned above (in 3.3.1) the $\mathrm{J}_{3}$-fluorescence peak is Stokes-shifted even at very low temperatures. We attempt here and in the subsequent subsection to understand the reasons of this shifting. Two possibilities are discussed: the energy transfer between different segments of the chain (or chains) and the phonon dressing of an exciton on a particular chain segment.

In this respect the energy-selective methods (persistent spectral hole buming and fluorescence line narrowing) can give information about homogeneous line broadening due to exciton-phonon interaction, about electron-phonon spectrum of the system and the relative value of inhomogeneous broadening. The fundamental principles of these methods are described in Section 5. 
We found that it is possible to burn persistent holes in the absorption band of TDC $\mathrm{J}$-aggregates in the spectral range at least from 16220 to $16360 \mathrm{~cm}^{-1}$. The possibility of hole burming in the absorption band of aggregates suggests that this band is inhomogeneously broadened at low temperature.

The temperature dependence of the hole width (FWHM) is shown in Fig.10(3.3). One can see that the hole width is independent of temperature at $T<9 \mathrm{~K} A$ similar behavior of the hole width in the low temperature limit has been found for excitonic states in aggregates of pseudoisicyanine (PIC) [33] and polysilane polymer chains [34]. The hole width in such systems at low temperatures is apparently determined by the dephasing rate only $[33,34]$ and therefore is equal to twice homogeneous linewidth $\gamma$. Note that the limiting value of the hole width $\Gamma_{H}(T=0)=2 \gamma_{0}=3.3 \mathrm{~cm}^{-1}$, corresponding to the dephasing time $T_{2}=6.2 \mathrm{ps}$, tumed out to be one order of magnitude greater in our case than that of PIC $\Gamma$ aggregates [33]. It is difficult to conceive that the fluorescence decay time of our system at $T<9 \mathrm{~K}$ is as short as $3.1 \mathrm{ps}\left(\mathrm{T}_{1}=\mathrm{T}_{2} / 2\right)$ (We measured the lifetime at $77 \mathrm{~K}$ to be ca. $40 \mathrm{ps}$ ).

The homogeneous line broadening of excitonic transition can be in general due to radiative or radiationless transitions to the ground state, trapping at an impurity and (or) self-trapping, scattering by phonons and impurities. The temperature broadening of the hole width of J-aggregates of TDC is well described by direct processes only with single phonon absorption and emission:

$$
\Gamma_{H}=a n+b(n+1)
$$

where $n$ is the phonon occupation number,

$$
n=[\exp (\mathrm{h} \omega / \mathrm{kT})-1]^{-1}
$$

$a$ and $b$ - exciton-phonon coupling coefficients. It was found that $a \approx b=3.3 \mathrm{~cm}^{-1}$ and $\omega=$ $19 \mathrm{~cm}^{-1}$. The equality of the coefficients $a$ and $b$ is rather interesting result. It implies the density of excitonic states by $\sim 20 \mathrm{~cm}^{-1}$ below and above the burning frequency near the absorption maximum is of the same value. A system of equidistant levels belonging to a homogeneous spectrum appears in the case of exciton coupling to an effective phonon mode with pronounced shift of equilibrium positions of atoms in the excited and ground states [35]. The model with equidistant exciton-phonon levels could explain the equality of coefficients $a$ and $b$ in (1) and the Stokes shift in our case. As the temperature broadening of the homogeneous line of J-aggregates is well described only by direct processes the 


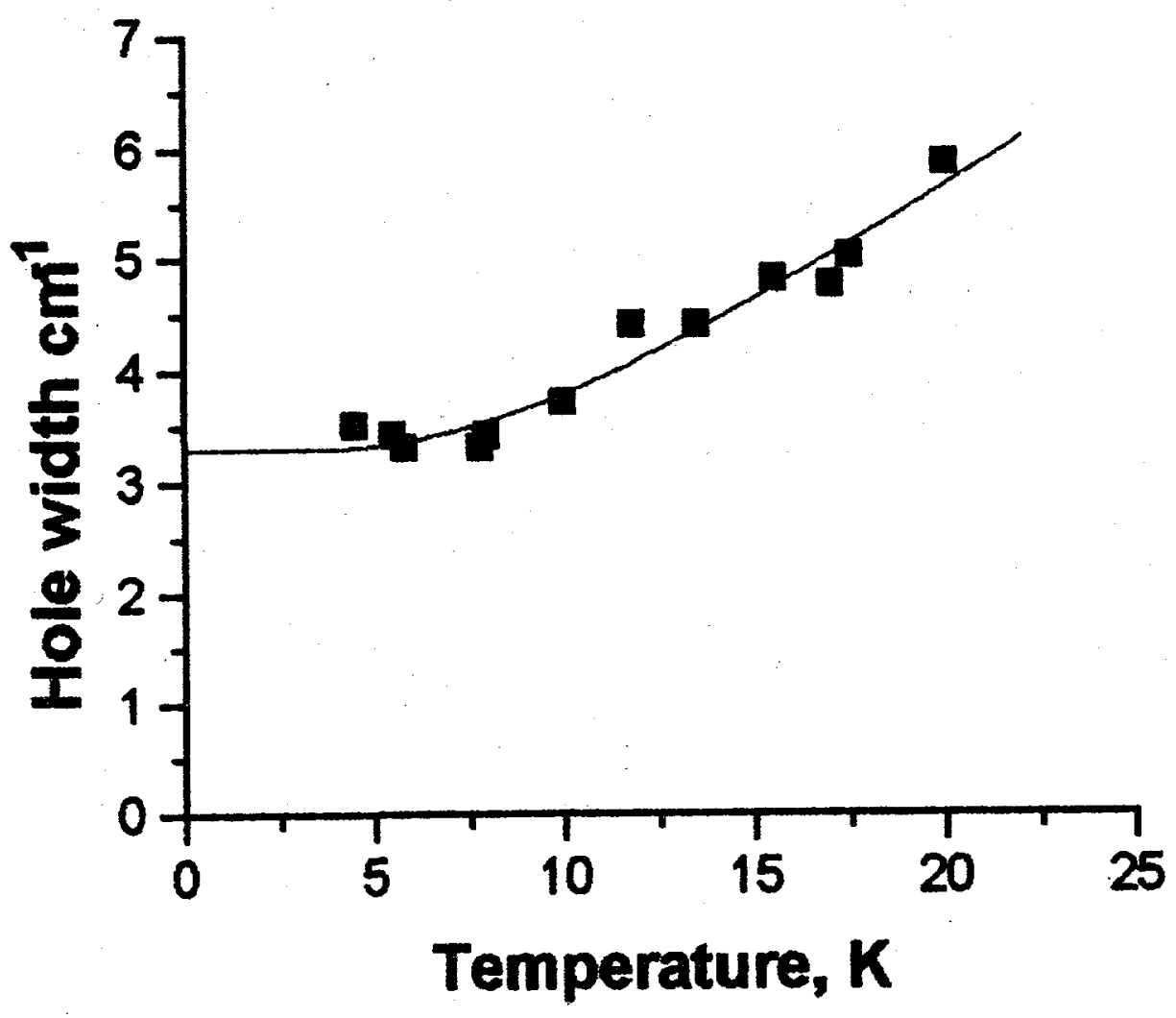

Fig.10(3.3). Temperature dependence of the spectral hole width (FWHM) burnt in the absorption J-band. 
temperature-independent linewidth is determined by spontaneous one-phonon emission rather than by the relaxation to the ground state. The relaxation with phonon emission can originate from optically populated exciton levels to the lower-lying levels of excitons coupled with phonons.

The J-aggregates spectrally and dynamically most closely related to TDC are those of an another thiacarbocyanine dye (TC), investigated in [7]. The dephasing time of $6.4 \mathrm{ps}$ at the low-temperature limit was measured near the absorption band maximum by the photon echo method. This value is in excellent agreement with that estimated from our hole burning data. As in our case, for TC J-aggregates having pronounced Stokes shift, the low-temperature dephasing rate was suggested to be determined by direct processes with one-phonon emission [7]. On the other hand, the residual homogeneous linewidth in the case of PIC J-aggregates in frozen solutions is found to be determined by the fluorescence decay to the ground state [33]. The characteristic property of the latter system is the absence of Stokes shift and very narrow J-bands (FWHM $=30-60 \mathrm{~cm}^{-1}$ ) which look almost exceptional among other J-aggregates.

Fig.2(3.3) shows the fluorescence spectrum of $\mathrm{J}_{3}$-form of TDC, obtained upon selective laser excitation and the absorption spectrum at $5 \mathrm{~K}$. A set of such a fluorescence spectra, obtained at different excitation laser frequency is shown on Fig.11(3.3). Following prominent features stand out from these figures:

1. An appreciable (compared to the bandwidth) Stokes shift between maxima of absorption and fluorescence spectra is observed.

2. The fluorescence band is narrower than the absorption band.

3. The fine structure in fluorescence spectrum is absent at selective excitation.

4. The fluorescence spectrum is virtually independent of the excitation frequency in the range studied. Only a slight increase in the red tail intensity is observed as the excitation frequency rises.

Note that in a widely studied J-aggregates of pseudoisocyanine (PIC) no Stokes shift was observed in frozen solutions at low temperatures [36]. A presence of the Stokes shift in our case, as well as in other J-aggregate systems [7,22a,36,37] can be accounted for by different exciton relaxation processes. As it has been mentioned above, it can be caused by the exciton self-trapping. This leads to the appearance of the Stokes shift which gradually increases from 0 with increasing of exciton-phonon coupling in 1D-systems [14b]. Therefore this implies very small exciton-phonon coupling)in the PIC J-aggregates as compared to TDC J-aggregates. Another possibility is the excitation energy transfer between chain segments of different lengths, leading to population of longest segments during excitation lifetime. This mechanism was proposed by Fidder and Wiersma [7] to 


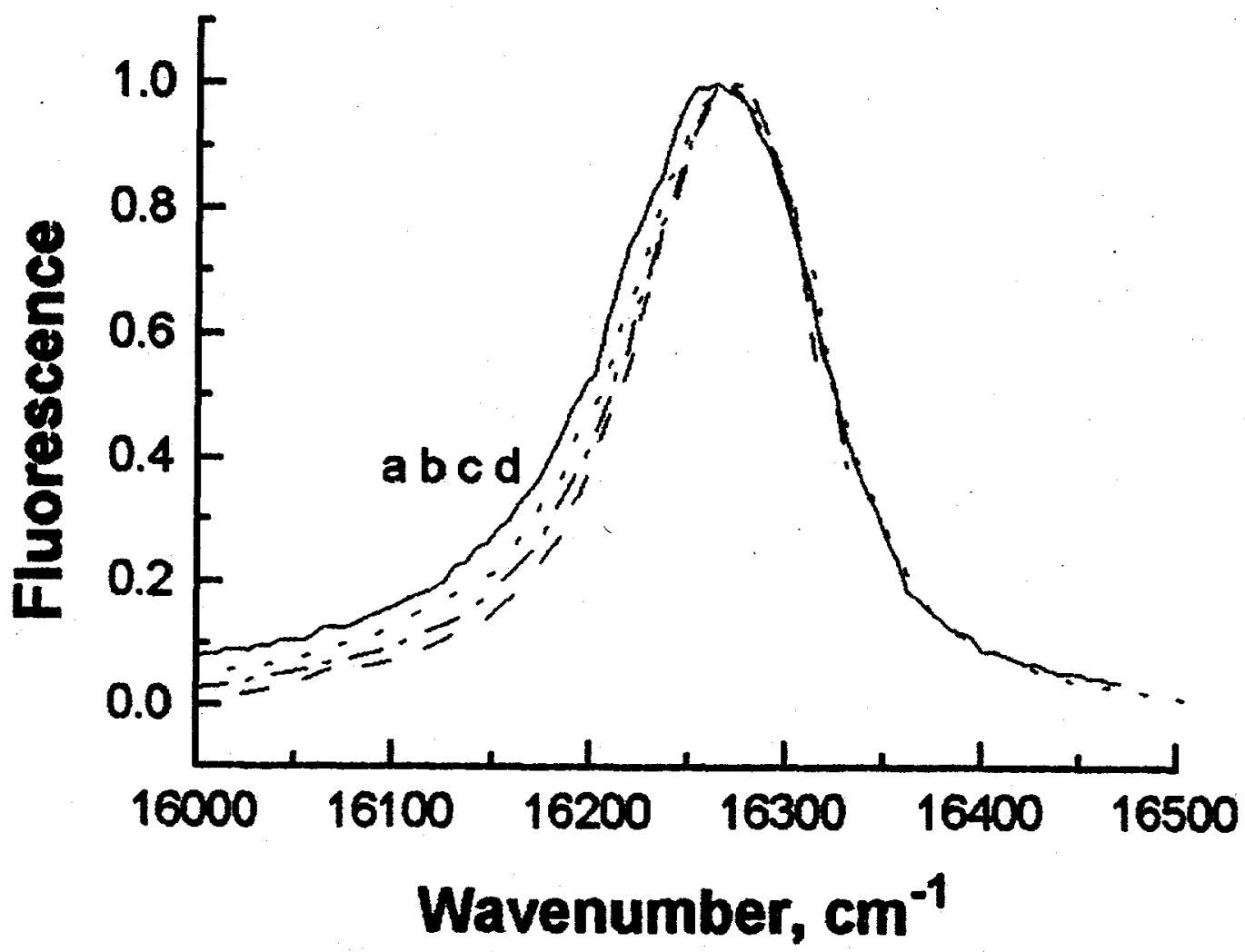

Fig.11(3.3). The dependence of the fluorescence spectrum on the laser excitation wavelength equal to $578 \mathrm{~nm}(\mathrm{a}), 590 \mathrm{~nm}$ (b), $612 \mathrm{~nm}$ (c) and $613 \mathrm{~nm}$ (d) at $6 \mathrm{~K}$. 
explain similar fluorescence band narrowing in J-aggregates. As both processes can contribute to the observed Stokes shift we can estimate an upper limit of the value of dimensionless exciton-phonon parameter $g$ from calculations of Higai and Sumi [14b]. Taking a value of half-width of excitonic band for $J_{3}$-aggregate of $B=1400 \mathrm{~cm}^{-1}$, estimated as a difference between the monomer and $J_{3}$-band at $5 K$ in WEG, and the maximum Stokes shift of ca. $140 \mathrm{~cm}^{-1}$ (measured at $20 \mathrm{~K}$ ) we can obtain $\mathrm{g}_{\max }=0.42$ from Fig 4 of Ref. 14b. Using this value of $g$ we can obtain the lattice relaxation energy gained in the case of complete localization of exciton on single site $\mathrm{S}=\mathrm{gB}=600 \mathrm{~cm}^{-1}$.

It is somewhat surprising that the trapped state has a sufficiently narrow fluorescence band with a rather small spectral shift (some $100-140 \mathrm{~cm}^{-1}$ ) with respect to the free state. However, broad fluorescence and large Stokes shift are inherent only for 3D-systems where localized excitation tends to occupy only single site $[11,27]$. On the other hand, the notions "free" and "self-trapped" excitons in low-dimensional structures become relative. In recent works of Sumi [14] it has been shown that while in strictly 1D lattice an exciton is always self-trapped it has in the case of small exciton-phonon coupling a large radius of delocalization and is described by Bloch waves. Sumi called this situation as weak self-trapping in a low-dimensional random lattice. Any Stokes shift (which tends to 0 as the coupling constant tends to 0 ) for this weak localized state is predicted in $1 D$ case [14b]. Thus, generally speaking, the excitonic states in low-dimensional systems can be only more or less localized ones.

An absence of the fine structure in luminescence spectrum at selective laser excitation together with possibility of narrow hole burning in absorption spectrum is at a glance rather unexpected. But narrow zero-phonon lines (ZPL) in luminescence spectra of isolated molecules are related usually to dispersionless local intermolecular vibrations; which can not reveal in aggregated species. Moreover, both effects of energy transfer selftrapping of exciton lead to disappearance of ZPLs in luminescence. For example, Toyozawa shown [12] that after self-trapping the ratio of one-to-zero phonon bands intensity increases by a factor of $(B / h \omega)^{2}$ equal to ca. $5 \times 10^{3}$ in our case if $\omega=20 \mathrm{~cm}^{-1}$. Note, that experimentally similar effect (the possibility of hole burning and the absence of ZPL in luminescence) has been observed earlier in other oligomer and aggregated photosynthetic systems [38-40].

The independence of the fluorescence band position of the excitation wavelength is more clear on the basis of fast energy transfer, but also does not exclude the STmechanism Similar behavior of the fluorescence spectrum has been observed for abovementioned aggregated systems $[39,40]$ and very recently for other J-aggregates in Langmuir-Blodgett films [41]. 
Increasing of the red tail intensity (Fig.11(3.3)) can be due to direct (or by-pass) self-trapping from highly-excited states (cf. analogous effect in Fig.8(3.3) at 77K for $\mathrm{J}_{2-}$ peak).

\subsubsection{Temperature Broadening of the Fluorescence Band}

Although the mechanism of relaxation is not well understood yet, we attempted to describe the temperature broadening of the fluorescence band by models, assuming either only dynamic disorder induced by phonons or both dynamic and static disorder. Because the red side of the fluorescence spectrum changes non-monotonically (see Fig.3(3.3)) we investigated the blue side half-width of the $J_{3}$-peak as a function of temperature. Fig.12(3.3) demonstrates this dependence together with the full width at half maximum of the absorption band. These two dependencies differ noticeably. The absorption FWHM is constant at low temperatures and begins to increase only at $\mathrm{T}>40 \mathrm{~K}$. This is in qualitative agreement with the model of Klafter and Jortner [5] involving both the static and dinamical disorder. At low temperatures the spectral width is determined only by static inhomogenities. On the other hand, the blue HWHM of the fluorescence peak increases continuously from $5 \mathrm{~K}$ with the negative second derivative. This suggests that static disorder does not prevail in the formation of the fluorescence spectrum even at very low temperatures.

To describe semi-quantitatively temperature broadening of the fluorescence spectrum semi-quantitatively we used the following model assumptions:

(1) The absorption spectrum observed is inhomogeneously broadened by static disorder

(2) Some relaxation process (energy transfer between different segments of the chain or dressing of the exciton resulting in weak localization) leads to the effect of vanishing of inhomogeneous broadening.

(3) The fluorescence $F(E)$ and absorption $A(E)$ spectra are related by an equation $[27,42]$

$$
F(E)=F_{0} A(E) \exp (-E / k T)
$$

where $F_{0}$ is a constant almost independent of $E$. Note that $A(E)$ corresponds to the relaxed system and does not coincide with the observed absorption spectrum. This equation is valid if the thermal distribution between excited state levels occurs during exciton lifetime, that is experimentally justified for our system $\left(T_{2}<6 \mathrm{ps}\right.$ and $\left.T_{1} \approx 40 \mathrm{ps}\right)$. 


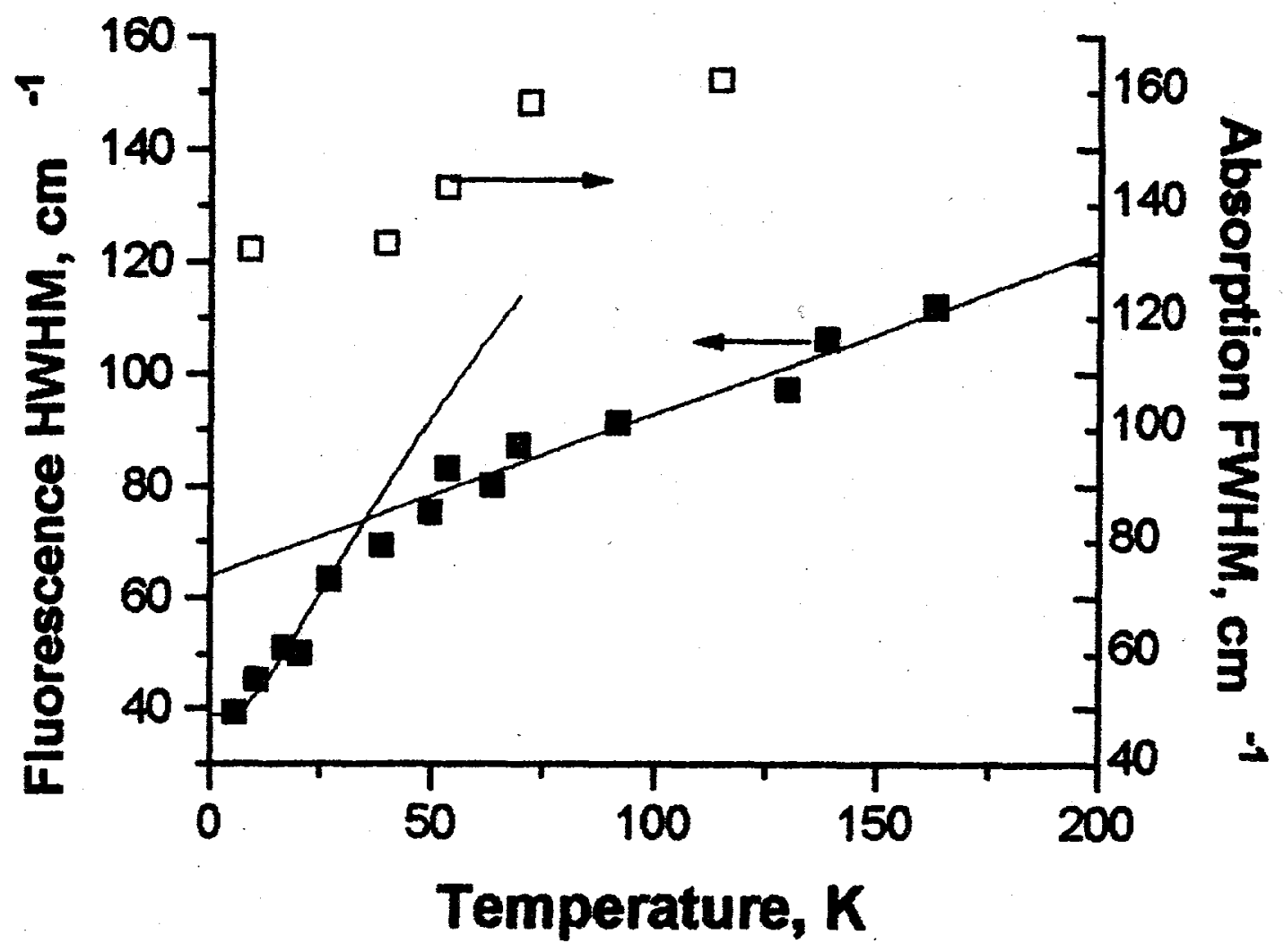

Fig.12(3.3). Temperature dependence of the blue side HWHM of the $J_{3}$ fluorescence peak and temperature dependence of the FWHM of absorption peak. Fitting of the fluorescence peak broadening is presented by Eqs. (4),(6) at low temperatures and by a straight line at high temperatures (see text). 
(4) The red side of the absorption spectrum $A(E)$ can be described by a Gaussian in the vicinity of maximum and by the exponential Urbach tail in the low-energy region.

The last two points result in a Gaussian fluorescence shape with the same halfwidth as the absorption $A(E)$ has in the vicinity of its maximum. Taking into account the above considerations, we attempt to justify the model elaborated by Schreiber and Toyozawa [8a] for effect of only dynamical disorder on absorption spectrum $A(E)$ by comparing temperature broadening of the observed fluorescence line with relations obtained in [8a]. In this theory the disorder parameter $D$ describes the fluctuations of onsite excitation energies, induced by a phonon with effective frequency $\omega$ and coupled linearly to an exciton

$$
D^{2}=\mathrm{S} h \omega \operatorname{coth}(\mathrm{h} \omega / 2 \mathrm{kT})
$$

In the strong scattering regime $(D>>B)$, the absorption line half-width is given by

$$
\Delta=\sqrt{2 \ln 2} D
$$

In the weak coupling case $(D<<B)$, taking into account the energy dependence of the density of states (DOS) in the area of absorption line, the authors [8a] obtained an approximate relation for the absorption line half-width which was proved by numerical calculations at high temperatures:

$$
\Delta \approx\left(\frac{\pi D^{2}}{\Gamma(d / 2)(2 \pi B)^{d / 2}}\right)^{\frac{2}{4-d}}
$$

where $d$ is the dimensionality of the system, $\Gamma(x)$ is the gamma function.

Thus, in the weak scattering limit and in the classical approximation $(T \gg \omega)$ the line broadens as $T^{\alpha}$ with $\alpha=2 / 3,1$, and 1.5 for $1 D, 2 D$ and 3D-lattices respectively.

We attempted to describe the observed dependence by exact equations (4) and (6) (or (5)). The limiting value of $\Delta$ at $T \rightarrow 0$ can be due only to zero-point quantum vibrations in this case. The best fit of experimental points in the range $6<\mathrm{T}<27 \mathrm{~K}$ by the function $\Delta=\Delta_{0}(\operatorname{coth}(\omega / 2 \mathrm{~T}))^{\alpha}$ gives $\Delta_{0}=40 \mathrm{~cm}^{-1}, \omega=19 \mathrm{~cm}^{-1} \alpha=0.66$ (see solid line in Fig.12(3.3) in the low temperatures region).

This implies that in the low temperatures range the fluorescence broadening is well described by model of Schreiber and Toyozawa in the weak scattering limit for 1D-lattice. It is interesting to note, that the same phonon frequency is involved in the process of exciton-phonon scattering measured from the temperature dependence of the hole 
broadening (see subsect. 3.3.4). From $\Delta_{0}$ value we can estimate $S=660 \mathrm{~cm}^{-1}$ using Eqs. (4) and (6) with $B=1400 \mathrm{~cm}^{-1}$. This $S$ value is closely related to the upper limit of $S$, found in the preceding subsection from the Stokes shift. This imply that the main contribution to the Stokes shift comes from the self-trapping process. The substitution of the parameters obtained to Eq. (4) gives $D<0.2$ in the temperature range studied, thus supporting the assumption about the weak scattering.

We attempted also to introduce the static disorder to the model. This has been accomplished by subtracting from experimental values $\Delta(T)$ a constant $\Delta_{s}$ using the equation $\Delta_{p}=\sqrt{\Delta^{2}-\Delta_{s}^{2}}$. It was found that no reasonable value of $\Delta_{s}$ provided the satisfactory fitting by Eqs. (4) and (6) (or (5)). Therefore, this suggests that the fluorescence spectrum is broadened only by the dynamic disorder induced by phonons in the weak scattering regime in $1 \mathrm{D}$-lattice.

At higher temperatures $\mathrm{T}=30-140 \mathrm{~K}$ the line broadening is not described by Eqs. (4) and (6) (or (5)). This can be due to the distortion of spectral shape and its deviation from pure Gaussian (possibly owing to the appearance of the $\mathrm{J}_{2}$-peak). Indeed, a comprehensive shape analysis showed that the blue side of the fluorescence spectrum can be described by Gaussian only at $\mathrm{T}<30 \mathrm{~K}$. At higher temperatures it is nearer to exponential function. At $\mathrm{T}>50 \mathrm{~K}$ the blue side half-width increases linearly with temperature (see Fig.12(3.3)). A possible reason for this is the shift of the fluorescence peak into the region of the exponential Urbach tail of the absorption spectrum (see below) as temperature increases. In this case, if Eq. (2) holds, fluorescence intensity will increase exponentially with decreasing energy $E$.

\subsubsection{The Long-Wavelength Tail of the Fluorescence Spectrum and Its Relation to the Urbach Rule}

It has been well established experimentally (see for review $[43,44]$ ) that the longwavelength tail of absorption spectra in many condensed systems can be described by exponential function:

$$
A(E)=A \exp \left(-\sigma \frac{E_{0}-E}{k T}\right)
$$

where $E_{0}$ is the energy near the absorption maximum, $A$ is the absorption at $E_{0}, \sigma$ is a dimensionless "steepness coefficient". Many theoretical works have been devoted to explain this rule. Exponential dependencies was proven for impurity centers, free and localized excitons, band-to-band transitions. All theoretical models give different 
temperature behavior of $\sigma$ at low temperatures dependent on particular physical system. The crucial point is a limiting value of $\sigma$ at $T \rightarrow 0$. It is evident that in an ideal dielectric crystal there should be no absorption below the energy of the band gap at $T=0$ and the absorption edge should be abrupt. This means that in a perfect crystal $\sigma \rightarrow$ const $\neq 0$ at $T \rightarrow 0$. At $T \neq 0$ transitions with phonon absorption can lead to the appearance of the long-wave tail below the absorption edge. Taking this into account it has been found that for localized excitations [45,46], free excitons [47] and band-to-band electron transition [48] in crystals the absorption tail has the exponential form (7) and $\sigma=\sigma_{0}+c T$ at low temperatures with $\sigma_{0} \approx 1$.

On the other hand, any structure imperfections (effect of impurities [49] or positional static disorder [50]) result in the exponential absorption tail even at $T=0$. Klafter and Jortner [50] showed that in 3D system the slope $\sigma / \mathrm{kT}$ is independent of temperature even at $\mathrm{kT} \geq \hbar \omega$ where $\omega$ is the frequency of a coupled-phonon. This implies that $\sigma \sim k T$ in a wide temperature range and therefore $\sigma \rightarrow 0$ as $\mathrm{T} \rightarrow 0$. Another possible mechanism accounting for the presence of the Urbach tail even at $T=0$ is a set of exciton-phonon transitions to the self-trapped state lying below the free exciton edge if the ST state exists [27,51]. This model gives, however, an exponential behavior only in the restricted energy region above the adiabatic minimum of the ST state. In this case also $\sigma \sim$ $\mathbf{k T}$, but in the region $\mathbf{k T} \ll \hbar \omega$. At high temperatures (kT $\gg \hbar \omega), \sigma=$ const for two and free dimensions and $\sigma \sim T^{\frac{1}{3}}$ for one dimension [8b]. (For subsequent analysis of validity of the Urbach rule in $1 D$-systems see $[52,53])$.

As for the fluorescence spectrum, if thermal equilibrium in the exciton - phonon system takes place, Eq. (3) holds and $F(E)$ in the tail region is described by [27]:

$$
F(E)=F \exp \left(-(\sigma-1) \frac{E_{0}-E}{k T}\right)
$$

An experimental evidence of equivalence of $\sigma$ values obtained from (6) and (7) independently was given in [54].

The detection of the red tail in huminescence in solutions of moderate optical density is more convenient than in absorption because of the greater signal-to-noise ratio in the former. Note that according to (8), as opposed to the absorption, there exist the exponential tail in the fluorescence spectrum at very low temperatures where $\sigma \rightarrow 1$.

The first experimental observation of the Urbach tail in several decades of the absorption tail of PIC J-aggregates in solution at room temperature was reported in [55]. Very recently Nabetani et.al have measured temperature dependencies of $\sigma$ in absorption 
and fluorescence in $\mathrm{J}$-aggregates embedded in the Langmuir-Blodgett film [41]. However, the data obtained from absorption spectrum cast some doubt because they are obtained only for one decade of the intensity decrease from a maximum where absorption spectrum can be described by a Gaussian (cf. [55]). Thus, a dependence $\sigma \sim \mathrm{kT}$ at low temperatures can mean the temperature independence of the Gaussian width at this temperature range which was established indeed [41]. On the other hand, the stepness coefficient $\sigma$ obtained from the fluorescence red tail, using Eq.(7) is well described by the relation $\sigma=1+c \mathrm{~T}$ at low temperatures and has not been interpreted by authors anyhow.

We observed the exponential fall in the fluorescence spectrum in the longer wavelength region than the $J_{2}$-peak and obtained the steppness coefficient in this region as a function of temperature using Eq. (7). This dependence is shown in Fig.13(3.3). One can see that it is similar to that of Nabetani et al. [41]. The linear asymptotic tending of $\sigma$ to 1 at $\mathrm{T} \rightarrow 0 \mathrm{~K}$ is very important evidence in favor of conclusion, made in the previous subsection of vanishing of the static disorder effect on fluorescence spectrum.

To describe our data quantitatively we used the simple model of Davydov and Lubchenko [45b], involving coupling of localized excitations with phonons. It gives the following temperature dependence of $\sigma$ :

$$
\sigma=1+\frac{k T}{\hbar \omega} \ln \left(\frac{E_{0}-E}{\operatorname{se} \hbar \omega(n+1)}\right)
$$

where $e$ is a base of natural logarithm, $\omega$ is the frequency of a coupled phonon in the ground electronic state, $s$ is the respective Huang-Rhys coupling factor, $n$ is the occupation number of a phonon of frequency $\omega$ given by (2). It is obvious that at low temperatures $\sigma$ is a linear function of $T$. It has been found [48] that taking into account of moving nature of excitation results at low temperatures only in a slight alteration of a constant factor in the argument of logarithm in (9): $\left(E_{0}-E\right) / \hbar \omega$ must be replaced by $\sqrt{ }\left(E_{0}-E\right) / \hbar \omega$. Therefore, it is rather difficult to distinguish between two cases. Putting $E_{0}-E=500 \mathrm{~cm}^{-1}$ we find by the best fitting procedure $\omega=110 \mathrm{~cm}^{-1}$ and $s=0.5 \mathrm{in}$ Eq.(9). The fitting curve with these parameters is shown in Fig.13(3.3).

As the degree of localization can not be determined from these data, the important conclusion, nevertheless, should be drawn that if the Eq. (3) holds, the spectra $A(E)$ and $\mathrm{F}(\mathrm{E})$ of relaxed excitons in molecular TDC J-aggregates are not affected by static disorder. 


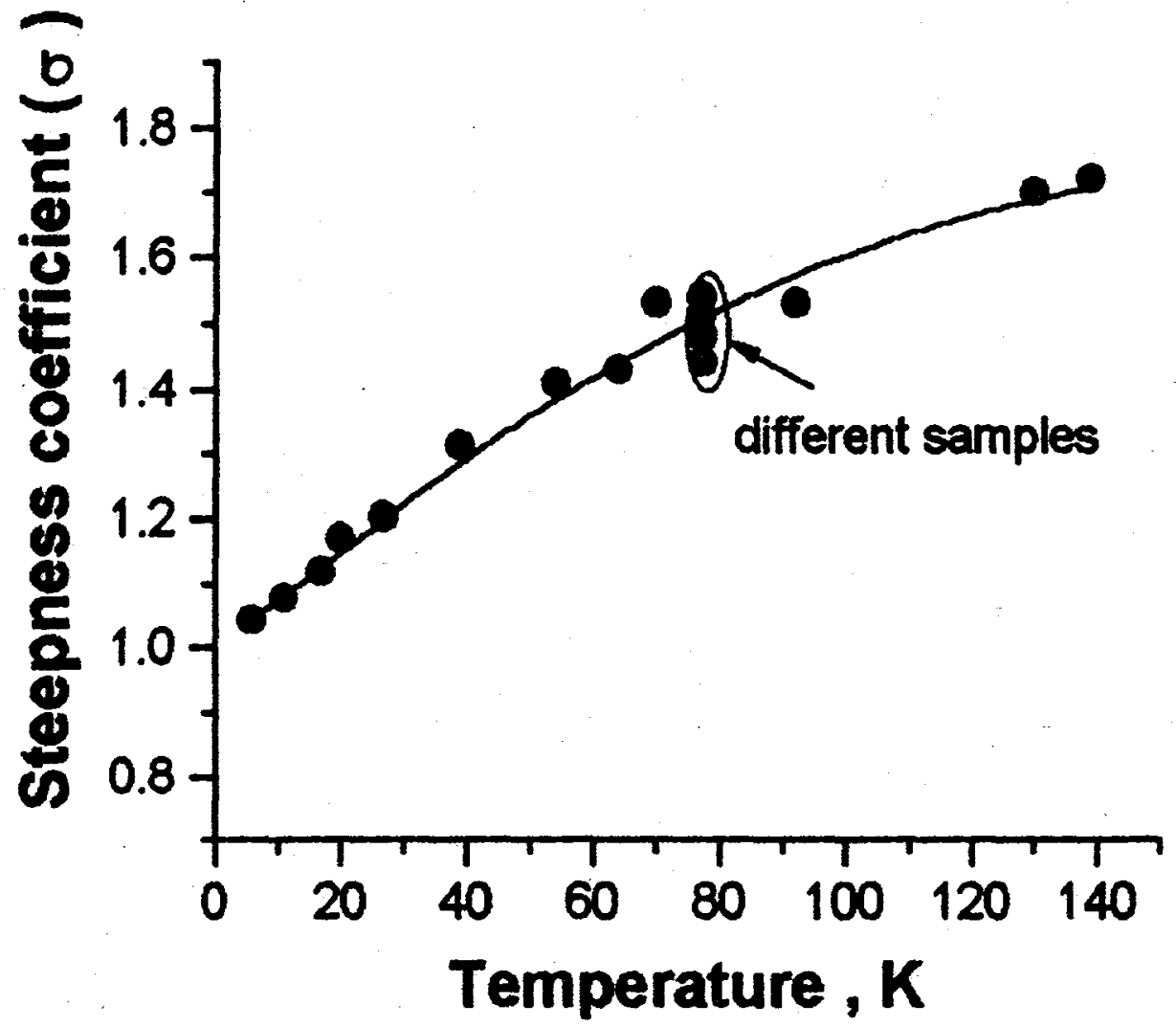

Fig.13(3.3). Temperature dependence of the steepness coeficient in the Urbach rule and its fitting by Eq.(9). 


\subsubsection{Conclusions}

Two types of J-aggregates of TDC have been studied at different temperature in frozen solutions.

It has been established for the first time that the short-wave peak $\left(J_{3}\right)$ posses a large negative value of the fluorescence anisotropy upon off-resonance excitation at $77 \mathrm{~K}$. This value is nearly equal to theoretical limit for randomly oriented emitters with perpendicular orientation of absorbing and emitting dipole moments. This implies the transitions to the top of the band (H-band) to be allowed that imposes some limitations on the molecular structure of this aggregate. Namely, the tilt angle of the monomer unit with respect to the chain direction must be large ( $\alpha>54.7^{\circ}$ for the staircase and $\alpha>45^{\circ}$ for the W-like structures respectively). It has been established also that the fluorescence anisotropy varies only slightly with increasing detection wavelength, replicating the red shifted $\left(J_{2}\right)$ peak in fluorescence. Thus, emission dipoles of $J_{3}$ and $J_{2}$ are highty correlated and the model of energy transfer between randomly oriented segments of one or different aggregates chains is excluded.

The observed non-monotonic temperature dependence of fluorescence spectrum is a strong indication against the energy transfer from $J_{3}$ to $J_{2}$ along the chain. The $J_{3}$ aggregate can rearrange to the $\mathrm{J}_{2}$-aggregate upon photoexcitation. This process is found to be temperature dependent, involving crossing the potential barrier of $20-30 \mathrm{~cm}^{-1}$ height. A decrease in the tilt angle from e.g. $60^{\circ}$ to $49^{\circ}$ is proposed as a possible mechanism of such a rearrangement. The disappearance of the $J_{2}$ fluorescence peak at elevated temperatures suggests that this process is possibly analogous to the self-trapping of exciton in deformable lattice.

Optical properties of the $J_{3}$ absorption and fluorescence band have been studied in more detail. The temperature dependence of the persistent spectral hole burnt in the absorption band can be described by direct one-phonon emission and absorption with equal effective coupling coefficients. At very low temperatures $\mathrm{T}<10 \mathrm{~K}$ the homogeneous linewidth is completely determined by phonon emission with the effective frequency of 20 $\mathrm{cm}^{-1}$. This leads to the conchusion that the $J_{3}$ fluorescence peak can be formed by clothed excitons. The dressing can be associated with the first step of trapping process.

A detailed study of the temperature broadening of the $\mathrm{J}_{3}$ fluorescence peak in comparison with the absorption peak, suggests the effect of vanishing of structural disorder under exciton relaxation. This effect can be attributed to the energy transfer between segments. On the other hand, the exciton-phonon coupling parameter, evaluated from the temperature dependence of the blue side of the $\mathrm{J}_{3}$ fluorescence peak is very close to that obtained, using model calculations of Higai and Sumi [14b] from the entire Stokes 
shift observed at low temperatures $\left(S \approx 600 \mathrm{~cm}^{-1}\right)$. Therefore, the Stokes shift it seems to be determined completely by exciton-phonon relaxation (dressing) resulting in the selftrapped state.

The exponential fall in the fluorescence intensity with energy has been established in the long-wave spectral range. The low-temperature value of the steepness parameter $\sigma$ of the Urbach rule indicates also the effect of vanishing of the static disorder during the exciton relaxation. The temperature dependence of $\sigma$ is well described by the model of localized excitation in a perfect crystal.

\section{References}

1. E.E. Jelley, Nature 138 (1936) 1009.

2. G. Scheibe, Angew. Chem. 49 (1936) 563.

3. G. Scheibe, Lage, Intesitat und Struktur von Absorptionbanden, in: Optische Anregungen Organischer Systeme, ed. W.Foerst (verlag Chemie, Wienheim, 1966) p. 109.

4. V. Sundström, T.Gillbro, RA. Gadonas, and A. Piskarkas, J.Chem.Phys. 89 (1988) 2754.

5. J. Klafter and J. Jortner, J.Chem.Phys. 68 (1978) 1513.

6. H. Fidder, J. Knoester, and D.A. Wiersma, J.Chem.Phys., 95 (1991) 7880.

7. H. Fidder and D.A Wiersma, J. Phys. Chem. 97 (1993) 11603.

8.(a) M. Schreiber and Y. Toyozawa, J. Phys. Soc. Jpn. 51 (1982) 1528, (b) ibid. 1544.

9. E.I. Rashba, Optika Spektr., 2 (1957) 88 (in Russian).

10. Y. Toyozawa, Progr. Theor. Phys. 26 (1961) 29.

11.Y. Toyozawa and Y. Shinozuka , J. Phys. Soc. Jpn 48 (1980) 472.

12. Y. Toyozawa in: Springer series in solid-state sciences, Vol. 49. Organic molecular aggregates. Electronic Excitations and Interaction Processes, eds. P. Reineker,

H. Haken and H.C. Wolf (Springer, Berlin, 1983) p. 90.

13. E.I. Rashba, Synthetic Metals, 64 (1994) 255.

14(a). H. Sumi and A. Sumi, J.Phys.Soc.Jpn. 63 (1994) 637.

(b) S.Higai and H.Sumi, ibid. 4489.

15. B. Pertzsch and U. Rössler, Solid State Commun. 37 (1981) 931.

16. U. Rössler and H. Yersin, Phys. Rev. B 26 (1982) 3187.

17. A. H. Matsui: in Excitonic Processes in Condensed Matter, Proc. SPIE - Int. Soc. for 
Opt. Engin. Ed. J. Singh, Darwin, 1994, Vol 2362, p. 376.

18. H.Tanino, W.W.Ruhle and K.Takahashi, Phys.Rev. B38 (1988) 12716

19. M. Furukawa et al. J. Phys. Soc. Japan. 58 (1989) 2976.

20. T. Kobayashi Excitonic Processes in Condensed Matter, Proc. SPIE / Ed. J. Singh, Darwin, 1994, Vol. 2362, p.19

21(a) Yu.V. Maliukin and O.G. Tovmachenko, Pisma JETP, 58 (1993) 385.

(b)Yu.V.Maliukin, V.P.Seminozhenko and O.G.Tovmachenko, JETP, 107 (1995) 812.

22(a) V.F. Kamalov, I.A. Struganova and K. Yoshihara, Chem. Phys. Lett. 213 (1993) 559.

(b)V.F. Kamalov, I.A.Struganova, T. Tani and K. Yoshihara, ibid. 220 (1994) 257.

23(a) H.Hada, C.Honda and H. Tanemura, Photogr.Sci.Eng., 21 (1977) 83

(b) C.Honda and H.Hada, ibid. 91.

24. M.D.Galanin, Proc. P.N.Lebedev Physical Institute, 5 (1950) 339 (in Russian).

25. H.Shinohara and M.Kotani, Bull.Chem.Soc.Jpn., 53 (1980) 3171.

26. M.J.Davies, A.C.Jones, J.O.Williams and R.W.Munn, J.Phys.Chem. 87 (1983) 541.

27. H.Sumi and Y.Toyozawa, J.Phys.Soc.Jpn., 31 (1971) 342.

28. E.I.Rashba, Opt. Spectr., 2 (1957) 568 (in Russian).

29. G.Scheibe, Angew.Chem. 52 (1939) 633.

30. E.G.McRae and M.Kasha, J.Chem.Phys. 28 (1958) 721.

31. V.Czikkely, H.D.Forsterling and H.Kuhn, Chem.Phys.Lett. 6 (1970) 11.

32. C.Reich, W.D.Pandolfe and G.R.Bird, Photogr.Sci.Eng. 17 (1973) 334.

33. R.Hirschmann and J.Friedrich, J.Chem.Phys., 91 (1989) 7988.

34. A.Tilgner, H.P.Trommsdorff, J.M.Zeigler and R.M.Hochstrasser, J.Chem.Phys. 96 (1992) 781.

35. D.P.Craig, L.A.Dissado and S.H.Walmsley, Che.Phys.Lett. 46 (1977) 191.

36. H.Fidder, J.Terpstra and D.A.Wiersma, J.Chem.Phys., 94 (1991) 6895.

37. S. de Boer and D.A.Wiersma. Chem.Phys., 131 (1989) 135.

38. J.Pahapill, Proceedings of the Academy of Sciences of the Estonian SSR. Physics Mathematics, 35 (1986) 416 (in Russian).

39. W.Kohler, J.Friedrich, R.Fisher and H.Scheer, Chem.Phys.Lett. 143 (1988) 169.

40. H. van der Laan et.al, Chem.Phys.Lett., 170 (1990) 231.

41. A.Nabetani, A.Tomioka, H.Tamaru and K.Miyano, J.Chem.Phys. 102 (1995) 5109.

42. V.M.Agranovich, Theory of Excitons, Moscow, Nauka, 1968 (in Russian).

43. M.V.Kurik, Phys.Stat.Sol, (a) 8 (1971) 9.

44. M.V.Kurik and L.I.Tsikora, Phys.Stat.Sol(b) 66 (1974) 695.

45(a) A.S.Davydov and A.F.Lubchenko, Doklady AN SSSR, 179 (1968) 1301 
(in Russian).

45(b) A.S.Davydov Phys.Stat.Sol. 27 (1968) 51.

46. A.V.Vinogradov, Fizika Tverdogo Tela 12 (1970) 3081.

47. M.G.Sceats and S.A. Rice, Chem.Phys.Lett. 25 (1974) 9.

48. V.D.Kagan, Soviet Physics-Solid State, 17 (1975) 2578 (in Russian).

49. V.L. Bonch-Bruevich, Phys.Stat.Solidi, 42 (1970) 35.

50. J.Klafter and J.Jortner, Chem.Phys. 26 (1977) 421.

51. K.Cho and Y.Toyozawa, J.Phys.Soc.Jpn., 30 (1971) 1555.

52. J.Ihm and J.C.Phillips, Phys.Rev. B 27 (1983) 7803.

53. M.Schreiber, Phys.Rev. B 34 (1986) 2914.

54. J.Takeda, T.Ishihara and T.Goto, Solid State Commun. 56 (1985) 101.

55. B.Kopainsky, J.K.Hallermeier and W.Kaiser, Chem.Phys.Lett., 87 (1982) 7.

\section{FLUORESCENCE PROPERTIES OF POLYMER-FULLERENE (POLYACETYLENE) MIXED FILMS}

\subsection{Introduction}

In the last years fullerenes attract attention of scientists as one of the most interesting from fundamental point of view and practically important materials [1-3]. Their spectral properties such as superconductivity $[4,5]$, photoconductivity $[6,7]$ and optical nonlinearity [8] were studied. The possibility was demonstrated of manufacturing photodiods, photovoltaic cells, different kinds of detectors and solar energy converters [2,3]. This work shows preliminary investigations of structure and electronic structure of fullerene C60 films obtained with the method of vacuum deposition, films based on fullerene $C_{60}$ - PA or fullerene $C_{60}$ - PPV compositions and films based on PA - PPV compositions. Bilayers of PPV film (donor)with fullerene $C_{60}$ film (acceptor) which form heterojuction interface and could be used as photodiodes or photovoltaic cells were also investigated.

\subsection{Structure and Optical Characteristics of Vacuum Deposited Fullerene $\mathbf{C}_{60}$ Films}

To obtain fullerene $C_{60}$ films, we used vacuum deposition method, which was thoroughly described in the report no. 1 . The powder of fullerene $C_{60}$ with purity of $99,5 \%$ was used without special cleaning. The heater for vacuum deposition was designed so that 


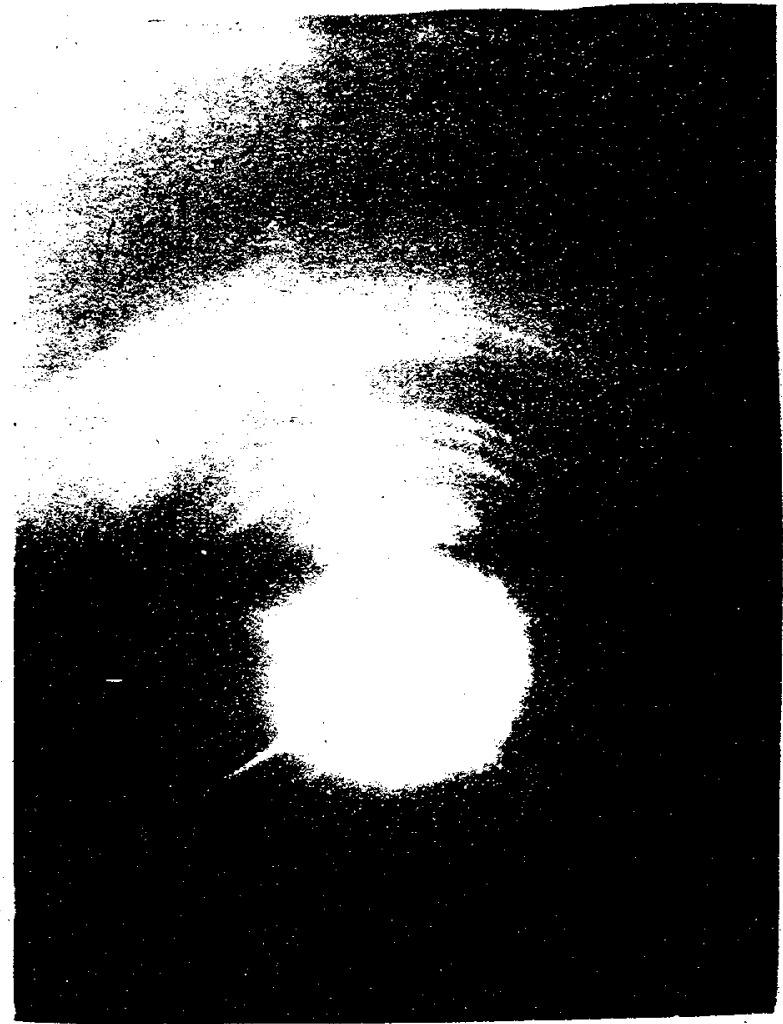

Fig.1(4.2) Reflection electron diffraction pattem of crystalline vacuum deposited fullerene $\mathrm{C}_{60}$ film. 
the substrate temperature decreased almost linear with increasing distance between substrate and fullerene $\mathrm{C}_{60}$ powder.

Our investigations showed that the appearance and crystal structure of fullerene $C_{60}$ films depend on the substrate temperature and the distance between the substrate and the $\mathbf{C}_{60}$ powder. Investigations of fullerene $\mathbf{C}_{60}$ films vacuum deposited on quartz substrates using the reflection electron diffraction showed that in broad range of temperatures and distances between $C_{60}$ film and the substrate amorphous fullerene $C_{60}$ films form. However, there is a narrow interval of the substrate temperature depending on reactor geometry in which crystalline $\mathrm{C}_{60}$ films form. In a standard reactor the conditions of evaporation are: evaporation temperature $600^{\circ} \mathrm{C}$, the substrate temperature $460^{\circ} \mathrm{C}$. Fig. 1(4.2) shows the reflection electron diffraction pattern of the crystalline fullerene $C_{60}$ film. Figure 1(4.2) shows nine Debae-Scherrer rings suggesting the high crystallinity of fullerene $\mathrm{C}_{60}$ films. The results are qualitatively the same as were obtained by the $\mathrm{X}$-ray diffraction for single fullerene $\mathbf{C}_{60}$ crystals [9].

Thus, the method of vacuum deposition allows one to obtain both amorphous and high crystalline fullerene $\mathrm{C}_{60}$ films.

It was shown early $[8,10]$ that the long-lived triplet emisson was not observed for fullerene $\mathrm{C}_{60}$ films at low excitation intensities. $A$ dramatic increase in the lifetime and the red shift and broadening of the spectrum is observed at high excitation densities. The luminescence intensity is seen to increase as the cube of the excitation intensity. Figures 2(4.2), 3(4.2), and 4(4.2) show photoluminescence spectra of amorphous fullerene $C_{60}$ films taken at different excitation wavelengths of an argon laser. No broadening of emission bands connected with nonlinear luminescence was observed in our experiments. However, the high intensity of photoluminescence for amorphous fullerene $C_{60}$ films without broadening of emission band can be connected with the formation of charge transfer complexes of $\mathrm{C}_{60}$ with oxygen.

\subsection{Electronic Structure of PPV - Fullerene $C_{60}$ Compositions}

The composites of Buckminsterfullerene $\mathrm{C}_{60}$ with conjugated polymers show very interesting photophysical phenomena [2,3]. It was shown that for conjugated polymers with nondegenerate ground states as donors, very fast photoinduced electron transfer from conjugated polymer to fullerene takes place, prior to either radiative or nonradiative recombination of photoexitations $[2,10]$. K.Yoshino and A.A. Zakhidov observed strong quenching of semiconducting polymer luminescence and interpreted the results by doping effect of $\mathrm{C}_{60}$ in the ground state [11]. However, doping of conjugated polymers with nondegenerate ground state is ruled out due to the absence of the electron spin resonance 


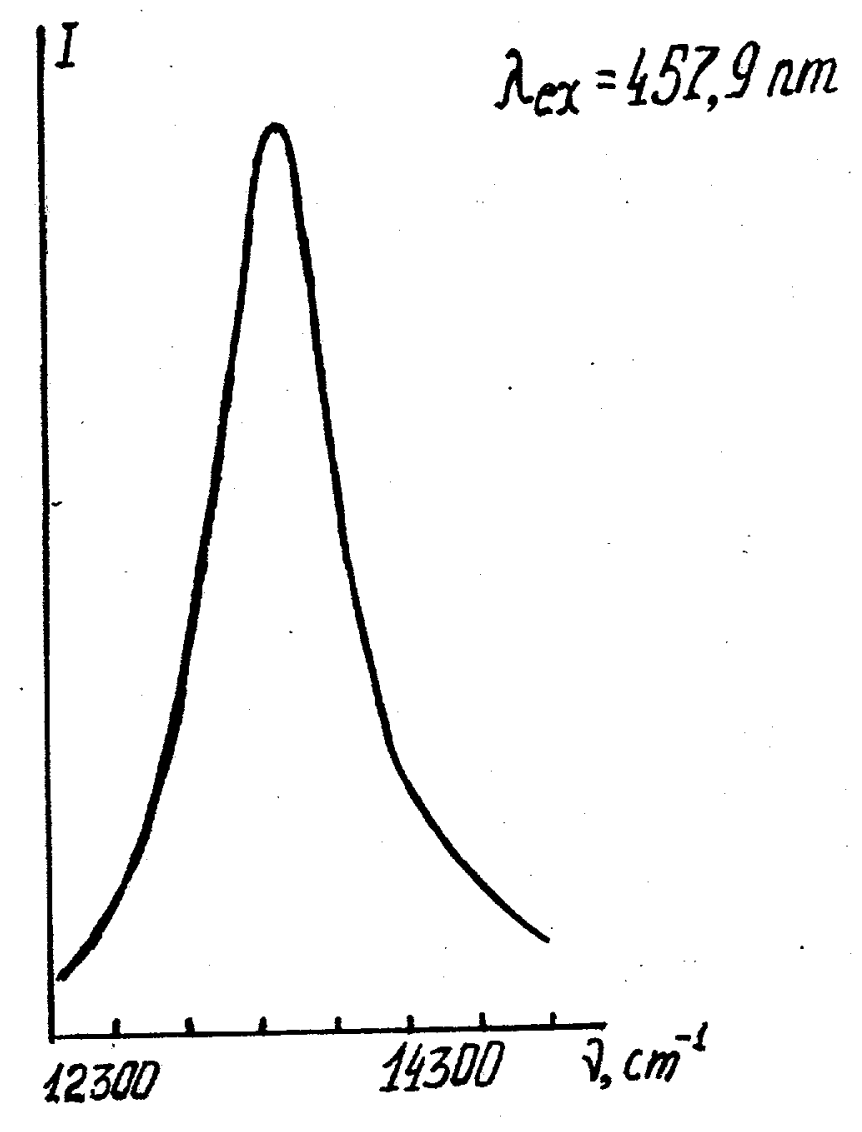

Fig.2(4.2) Photoluminescence of amorphous fullerene C60 films detected under excitation $457.9 \mathrm{~nm}$.

79 


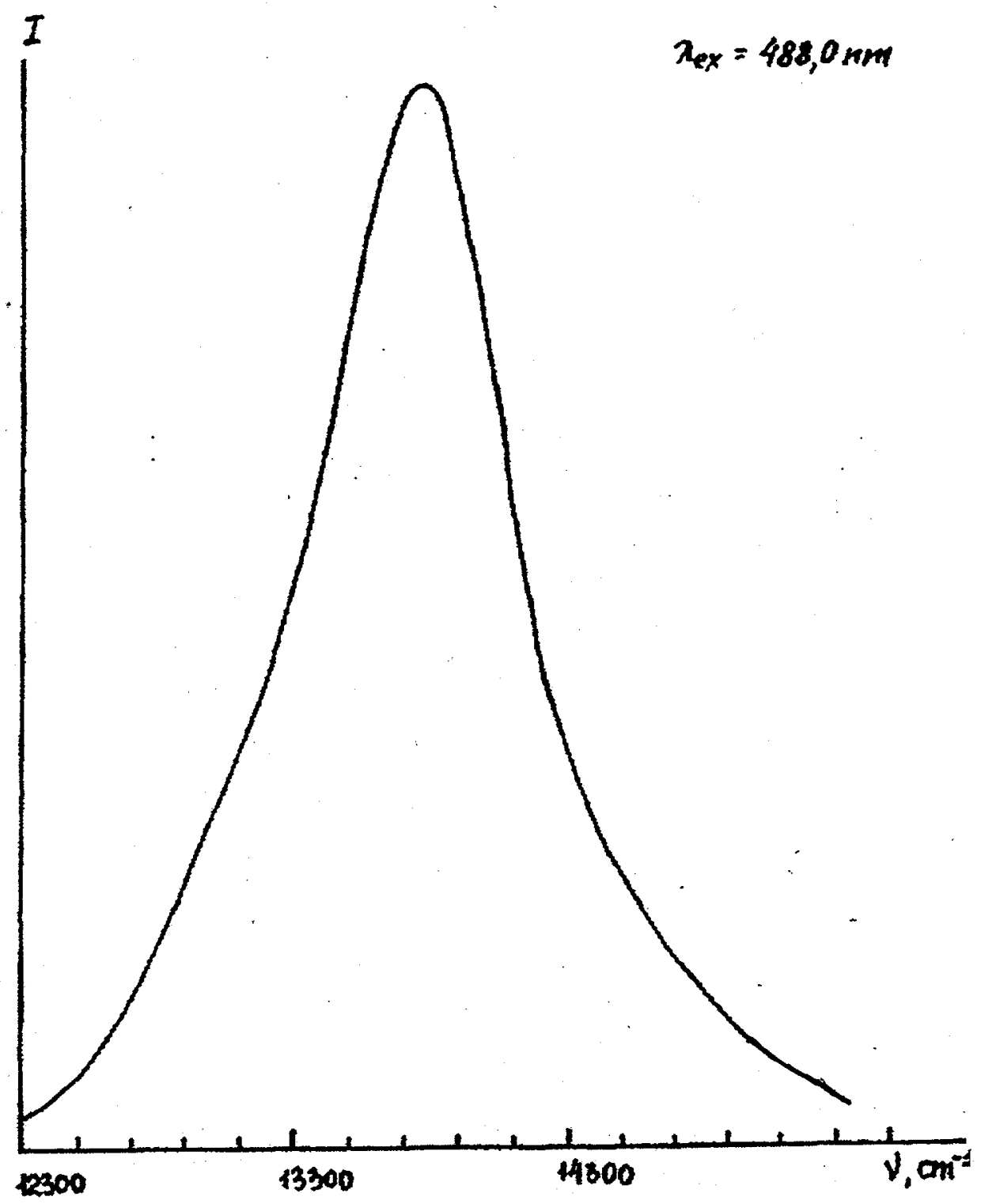

Fig.3(4.2) Photoluminescence of amorphous fullerene $C_{60}$ films detected under excitation $488 \mathrm{~nm}$. 


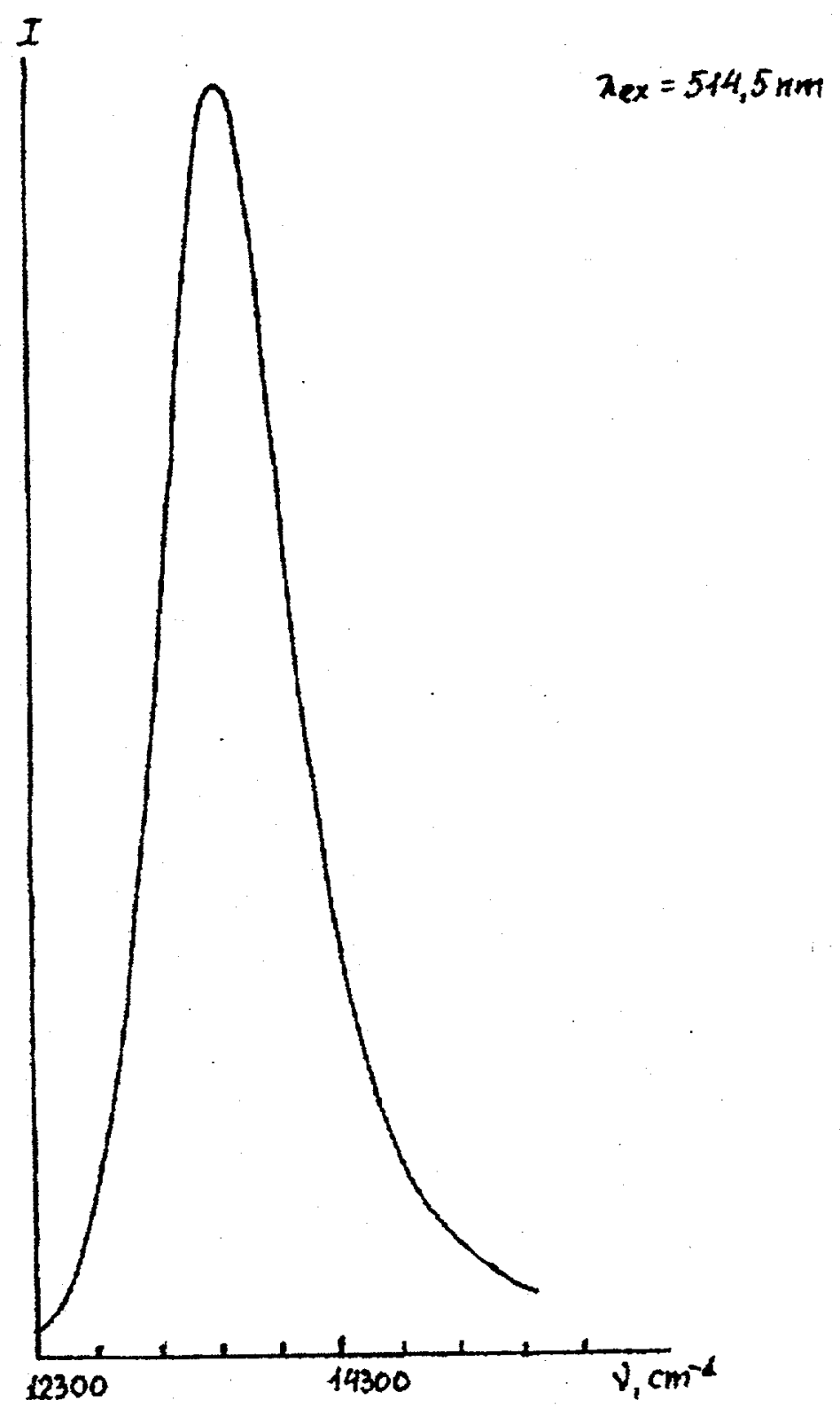

Fig.4(4.2) Photoluminescence of amorphous fullerene $C_{60}$ films detected under excitation $514.5 \mathrm{~nm}$ 
signal from the $\mathrm{C}_{60}$ anion in the ground state $[2,10]$. Therefore, the quenching of luminescence of conjugated polymers with nondegenerate ground state mixed with $\mathrm{C}_{60}$ supports the uitrafast, reversible photoinduced electron transfer from conjugated polymer to $\mathrm{C}_{60}$.We studied the photoluminescent properties of PPV films and mixtures of PPV with $\mathrm{C}_{60}$. Photoluminescent properties of PPV films were discussed in the Section 2.2 in Report no. 1 ( see, for example, Fig. 3(2.2), 5(2.2), 6(2.2) in Report no. 1). One can see from these figures that all forms of PPV films show high intensity of photoluminescence. The mixtures films of PPV with $\mathrm{C}_{60}$ were produced from solution. Films contain from $2 \%$ to $5 \%$ of fullerene $\mathrm{C}_{60}$. Our investigations show that films exhibit very weak photoluminescence. The" photoluminescence intensity of PPV decreases by a factor of 1000 in the presence of $2 \%$ fullerene $C_{60}$.

\subsection{Electronic Structure of Fullerene $\mathbf{C}_{60}$ - PA Composite Films}

It was shown earlier that photoinducted electron transfer is observed in conjugated polymers with nondegenerate ground state mixed with fullerene $C_{60}$. It can be observed by the techniques of photoinduced spectroscopy or photoinduced electron spin-resonance $[2,3,10,11]$. In the case of a conjugated polymer with the degenerate ground state as donor, no indications of photoinduced charge transfer to fullerene $\mathrm{C}_{60}$ were observed $[12,13]$. For investigation two different soluble polydiacetylene derivatives were used [12, 13].

In the present work we used soluble modification of polyacetylene for investigation. Previously we showed that PA we used is characterized by the absence of charged soliton bands and high stability of neutral solitons in photoinduced absorption spectra [14]. These investigations showed that the addition of $5 \%$ fullerene to that type of PA does not result in the appearance of bands characteristic for charged solitons, in photoinduced absorption spectra. Fig. 1(4.4) shows photoinducted absorption spectra of the PA film containing $2 \%$ fullerene. The figure shows the neutral soliton band and the absence of absorption characteristic of charged solitons. In opinion of Heeger, who observed the absence of the photoinduced electron transfer in the polydiacetylene fullerene $\mathrm{C}_{60}$ mixture, this is connected with the fact that the rate of the photoinduced formation of a neutral soliton in PA is greater then the rate of photoinduced electron transfer [13].

The PA - fullerene $\mathrm{C}_{60}$ mixture films were investigated with the IR and resonance Raman spectroscopy. Figure 2(4.4) and Fig. 3(4.4) show resonance Raman spectra of the PA film and of the PA film containing $5 \%$ of fullerene $\mathrm{C}_{60}$, respectively. Their comparison shows that the presence of fullerene $C_{60}$ has no effect on Raman spectra of PA 


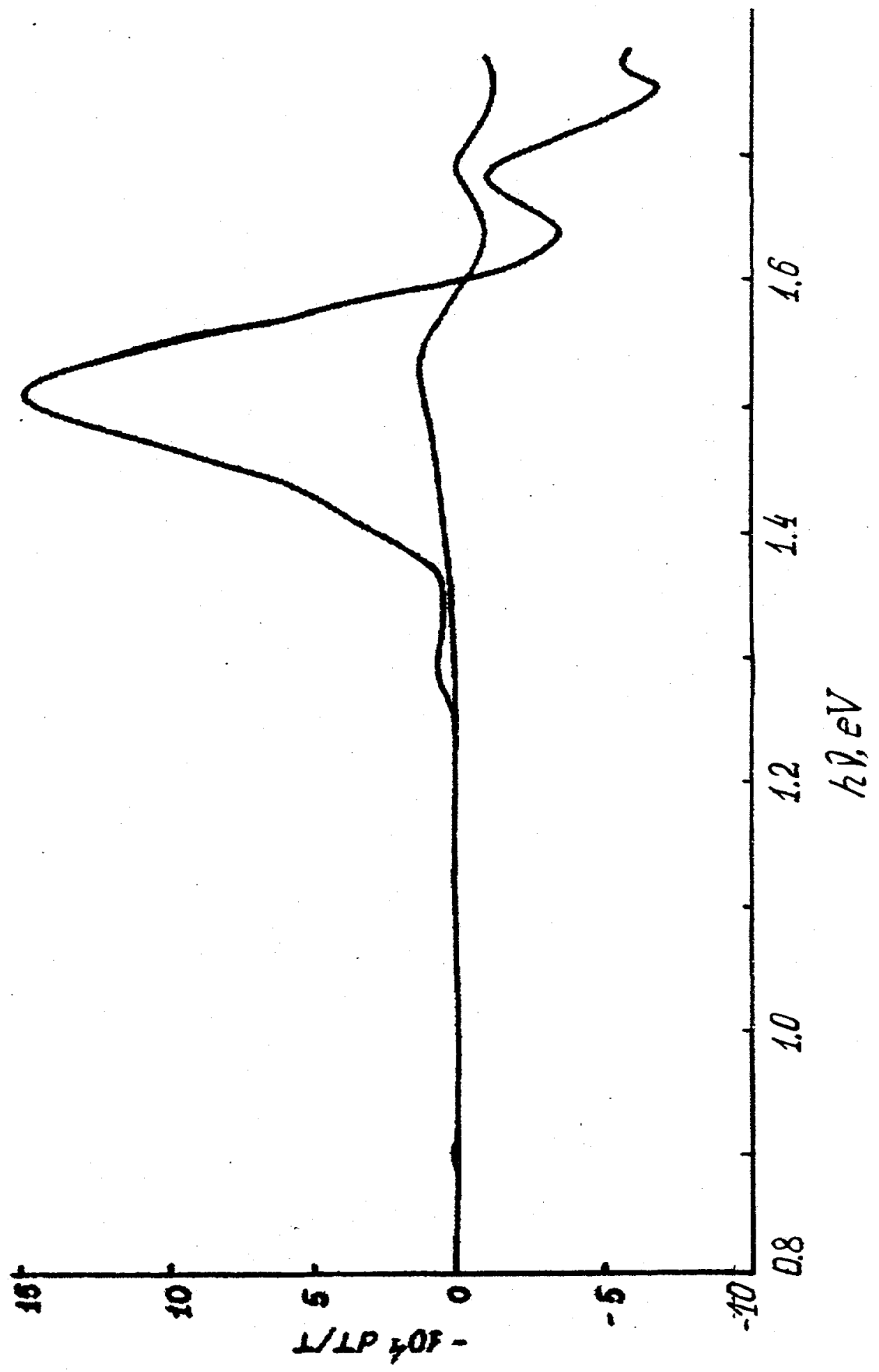

Fig.1(4.4) Photoinduced absorption spectrum of film from PA-PVB blend containing $2 \%$ fullerene $\mathrm{C}_{60}$. 


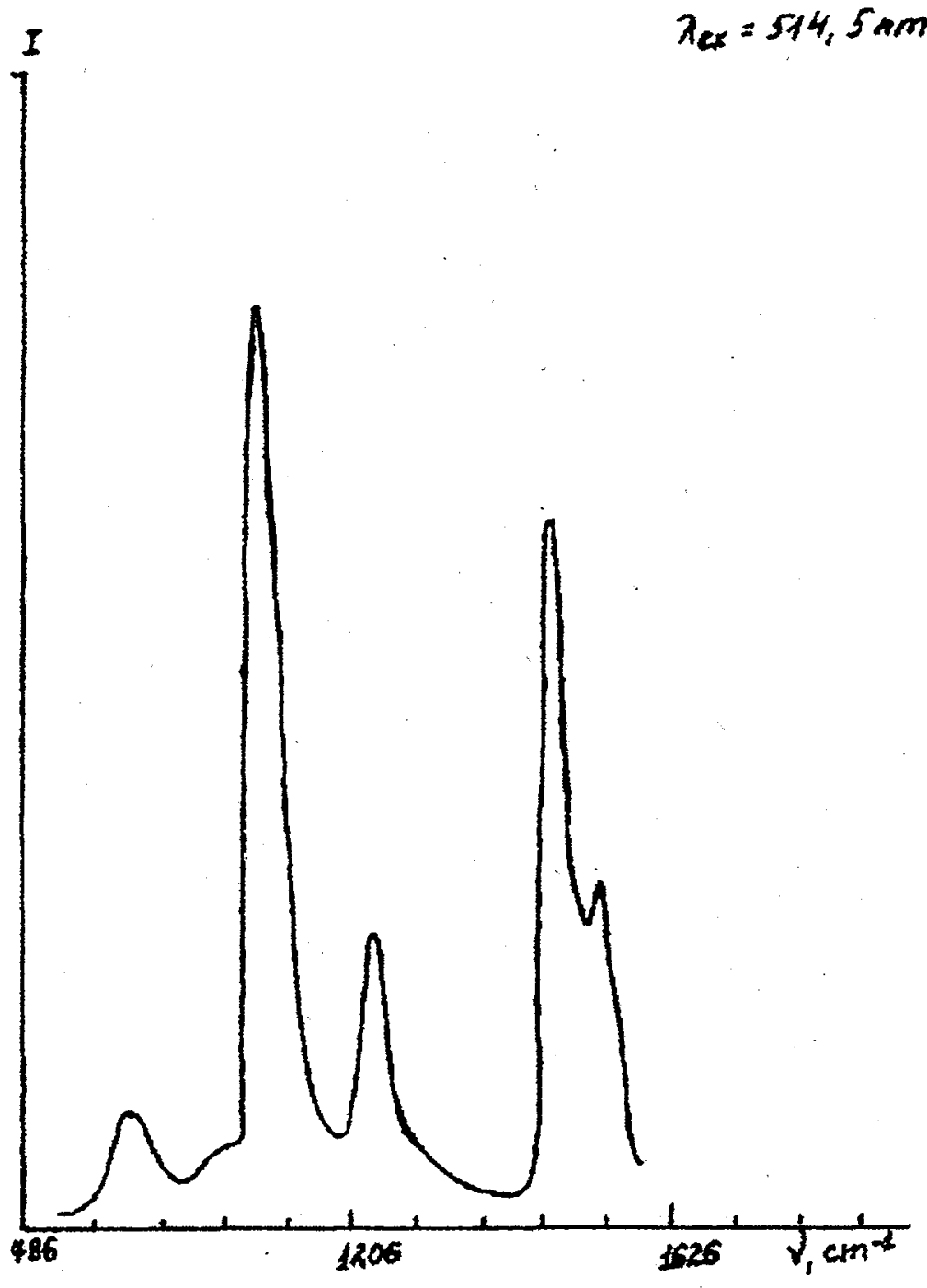

Fig.2(4.4) Resonant Raman scattering spectrum of PA-PVB blend. (excitation $514,5 \mathrm{~nm}$ ). 


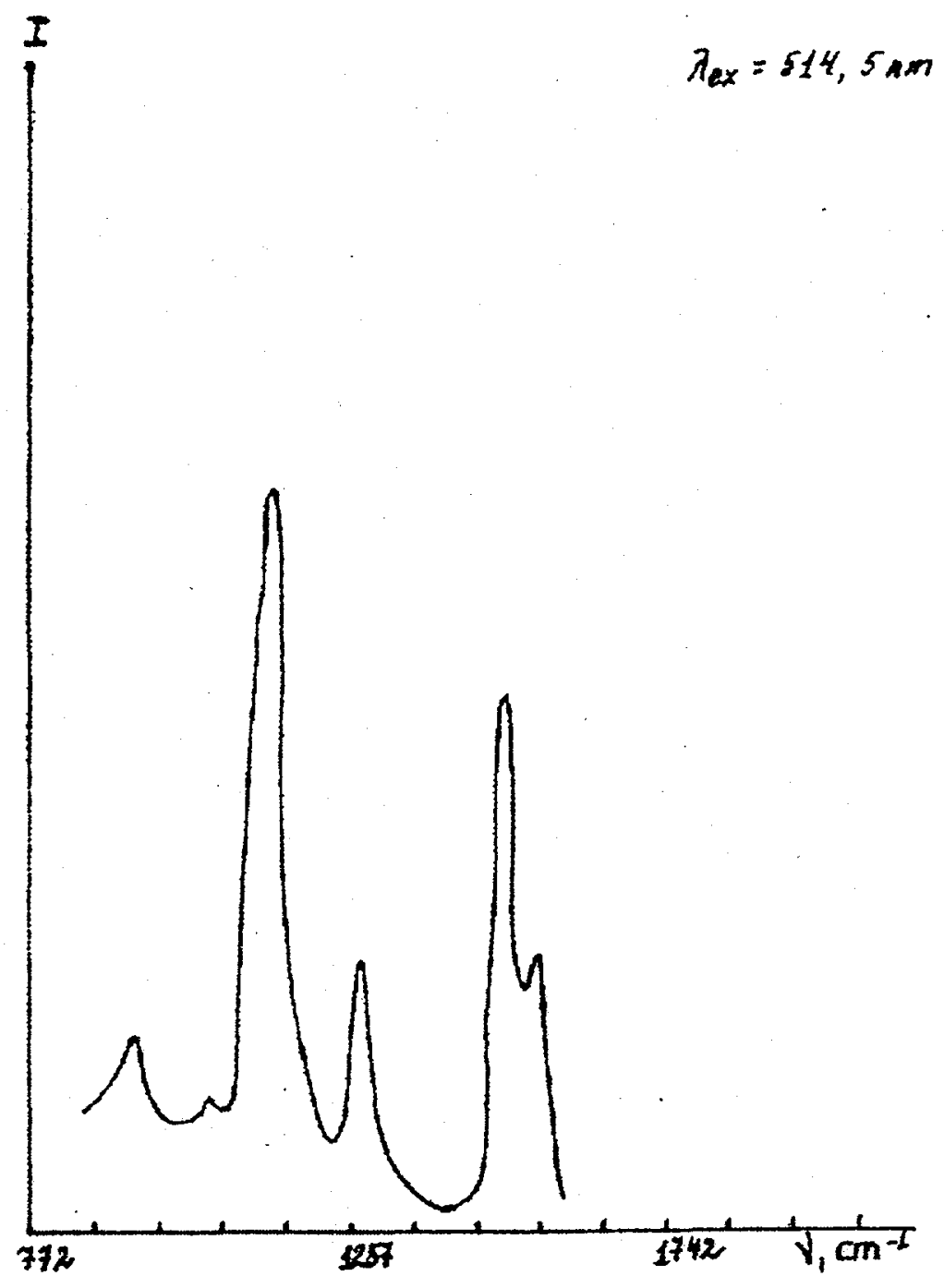

Fig.3(4.4) Resonant Raman scattering spectrum of PA-PVB blend containing \%5 of fullerene $C_{60}$. 


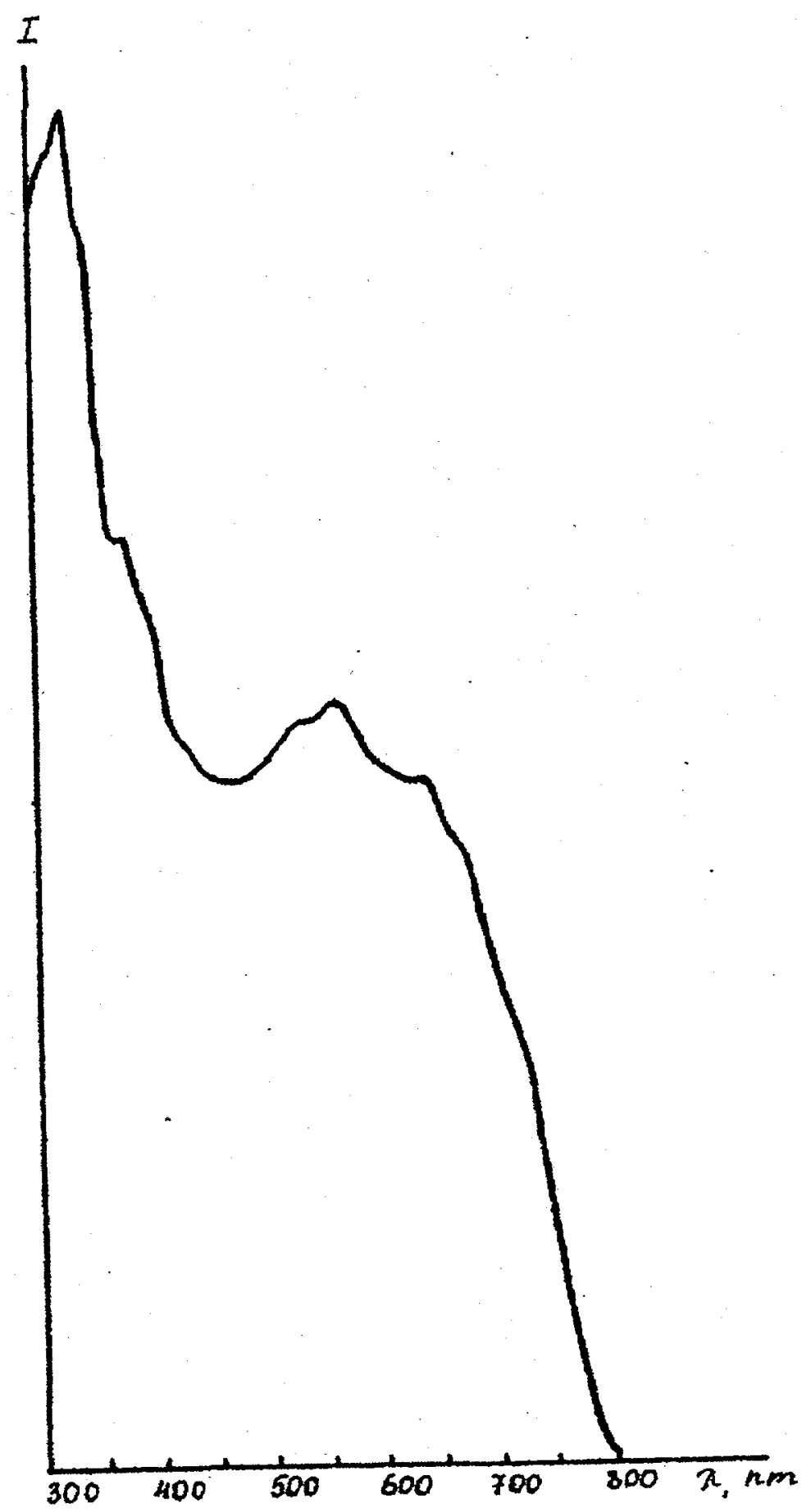

Fig.1(4.5) Absorption spectrum of PA-PPV blend in PVB matrix. 
We assume that the absence of the electron transfer is explained by the fact that photoexcited PA does not act as the electron donor for fullerene $C_{60}$.

\subsection{Electronic Structure of PPV - PA Mixtures}

Comparison of photoinduced optical properties of fullerene $C_{60}$ mixed with conjugated polymers with nondegenerate and degenerate ground states shows that photoinduced electron transfer are observed only for polymers with the nondegenerate ground state. For example, in the case of PPV, the efficient electron transfer to fullerene $\mathrm{C}_{60}$ during photoexcitation was observed. In the case of PA, the situation is opposite: no electron transfer to fullerene $\mathrm{C}_{60}$ was observed. We suppose that PA does not act as a donor for fullerene $\mathrm{C}_{60}$ upon photoexcited. We assume that the position of the level of a neutral soliton in PA may result in its ability to act as the electron acceptor to photoexcited polyconjugated polymers with nondegenerate ground state. In this case, for example, the electron transfer from PPV to PA should be observed during photoexcitation. To check this assumption, we prepared films containing PPV and PA with molar ratio of $1 / 1$. Figure $1(4.5)$ shows the typical absorption spectrum of the PAPPV mixture in a PVB matrix. One can see that the film contains PA and partly conjugated PPV in almost equal molar ratio. The absorption spectrum of partly conjugated PPV is shown in Fig. 2(4.5). Studies of photoluminescence of films obtained showed that PA is the exceptionally strong quencher of the PPV fluorescence. We failed to observe the PPV fluorescence even at very high exciting light intensity. These results are preliminary and suggest that the fluorescence quenching is connected with the electron transfer from PPV to PA during photoexcitation.

\section{References}

1. W.Kratschmer, L.Lamb, K.Fostiropoulos, D.Huffman, Nature, 347 (1990) 354.

2. N.S.Sariciftci, A.J.Heeger, Intern. J. of Modern Phys. B 8 (1994) 237-274.

3. N.Serdar Sariciftci Progr.Quant.Electr. 19 (1995) 131-159.

4. A.F.Hebard, M.J.Rosseinsky, R.C.Haddon, D.W.Murphy, S.H Glanum, T.T.M.Palstra, A.P.Ramizer, A.R.Kortan Nature 350, 600 (1992).

5. Proceedings of the Adriatico Research Conference, (World Scientific, Singapore, 1993) pp.383-445.

6. Y.Wang, Nature 356, 585 (1992).

7. R.Konenkamp, J.Erxmeyer, A.Weidinger, ApplPhys.Lett. 65, 758-760 (1994).

8. HJ.Byme, W.K.Maser, M.Kaiser, L.Akselrod, J.Anders, W.W.ruhle, X.Q.Zhou, A.Mittelbach, S.Roth, Applied Phys. A, Solids and Surfaces 57, 81-86 (1993). 


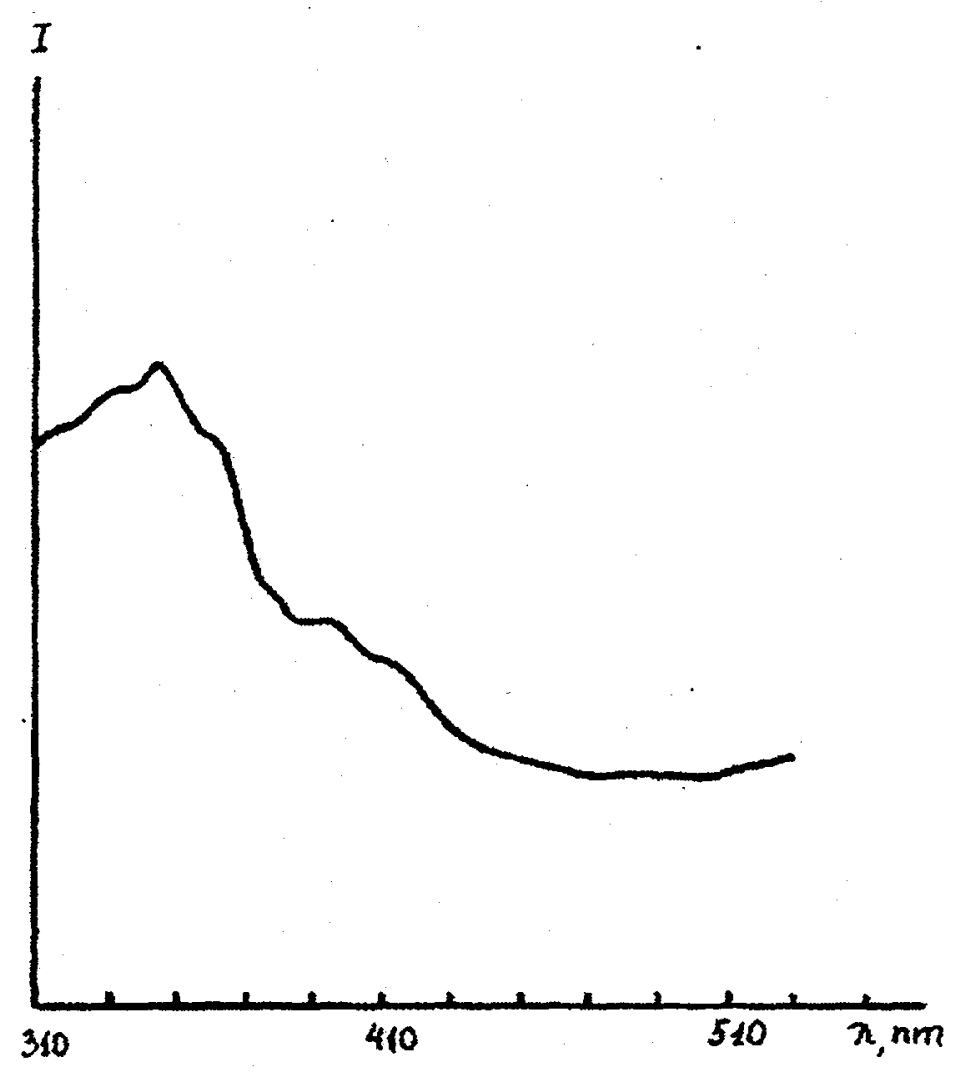

Fig.2(4.5) Absorption spectrum of PPV in PVB matrix.

88 
9. R.F.Xiao, W.Ho, L.Chow, K.K.Fung, J.Zheng, J.Appl.Phys., 77 (1995) 3572.

10. N.S.Sariciftci, L.Smilowitz, A.J.Heeger, F.Wudl, Science, 258 (1992) 1474.

11. S.Morita, A.A.Zakhidov, K.Yoshino, Solid State Commun., 82 (1992) 249.

12. L.Smilowith, N.S.Sariciftci, R.Wu, C.Gettinger, A.J.Heeger, F.Wudl, Phys.Rew., B 47, 13835-13842 (1993.

13. N.S.Sariciftci, B.Kraabel, C.H.Lee, K.Pakbaz, A.J.Heeger, D.J.Sandman, Phys.Rev., B 50, 12044-12051 (1994).

14. D.Yu.Paraschuk, T.A.Kulakow, V.M.Kobryanskii, Phys.Rev., B 50 (1994) 907.

\section{SELECTIVE LASER SPECTROSCOPY OF MOLECULES IN SOLIDS: MODERN ASPECTS}

We have used the techniques of fluorescence line narrowing and spectral hole burning to study optical properties of conjugated polymers in thin films and of Jaggregates of cyanine dyes in solutions and Langmuir-Blodgett films at room and liquid helium temperatures. These methods originally developed for studying homogeneous spectra of impurity molecules in solids in the presence of strong inhomogeneous broadening can also be applied for an investigation of energy transfer, spectral diffusion, and the dynamics of optical excitations in polymers and molecular aggregates (see Report 1). Below we discuss the general principles of selective spectroscopy of molecules in condensed phase.

\subsection{Introduction}

The techniques of fluorescence line narrowing (FLN) [1,2] and spectral hole burning (HB) $[3,4]$ are two variants of frequency- selective, high-resolution laser spectroscopy of solids based on selective excitation of molecules or ions with a narrowband laser. The selective excitation eliminates the inhomogeneous broadening of optical absorption and emission profiles and thus provides the information about the homogeneous spectra consisting of the narrow zero-phonon lines (ZPLs) accompanied by broad phonon sidebands (PSBs) [ 5 - 7].

The selective excitation can be achieved because of the existence of ZPLs in optical spectra, which have at low temperatures very small linewidths and high peak intensities. The homogeneous ZPLs cannot be observed using conventional broadband excitation since they are masked by the large inhomogeneous broadening caused by the 
statistical distribution of ZPL frequencies resulting from a matrix imperfection. Because the natural linewidth of a purely electronic ZPL for the allowed transition in the spectrum of polyatomic molecule with a typical fluorescence lifetime of several nanoseconds is of the order of $10^{-3} \mathrm{~cm}^{-1}$, while the inhomogeneous broadening in amorphous matrices is about $100 \mathrm{~cm}^{-1}$, monochromatic excitation allows one to increase the spectral resolution by five orders of magnitude. In the case of forbidden transitions involving long-lived metastable levels, the resolution can be increased in principle by several more orders of magnitude.

However, it is clear that in the general case the selective excitation of impurity centers via narrow ZPLs always occurs simultaneously with the nonselective excitation of other centers from an inhomogeneous ensemble through broad overlapped PSBs. Therefore, even highly monochromatic excitation does not allow one to eliminate completely the inhomogeneous broadening and to obtain a true homogeneous spectrum. This raises fundamental problems concerning the relation between the shapes of homogeneous luminescence (absorption) spectra and those of luminescence (hole) spectra recorded upon laser excitation, and of the excitation conditions that provide the maximum selectivity. These problems were investigated in detail in our papers [8 - 17].

In the following we present a brief review of our model studies of the mechanism of eliminating imhomogeneous broadening in the optical spectra of impurity centers upon monochromatic excitation resulting in FLN and $\mathrm{HB}$.

\subsection{Monochromatic Excitation of Luminescence of Molecules and Molecular Aggregates in Solids}

Consider an ensemble of impurity centers in an inhomogeneous matrix at low temperature. We assume that the concentration of the centers is low so that they do not interact with one another. The homogeneous absorption $A\left(v-v_{0}\right)$ and luminescence $E\left(v-v_{0}\right)$ spectra in the region of an electronic transition (purely electronic or vibronic) can be written in the form [18]

$$
\begin{aligned}
& A\left(v-v_{0}\right)=\alpha_{1} z_{1}\left(v-v_{0}\right)+\left(1-\alpha_{1}\right) p_{1}\left(v-v_{0}-\Delta_{1}\right), \\
& E\left(v-v_{0}\right)=\alpha_{2} z_{2}\left(v-v_{0}\right)+\left(1-\alpha_{2}\right) p_{2}\left(v-v_{0}+\Delta_{2}\right),
\end{aligned}
$$

where $\alpha \mathrm{Z}$ and $(1-\alpha) \mathrm{p}$ are ZPL and PSB, respectively, $\alpha$ is the Debye-Waller factor, $v_{0}$ is the frequency of the ZPL maximum and $\Delta$ is the Stokes shift (the distance between the 
ZPL and PSB maxima). The integrals of all the functions $A, E, z$, and $p$ are normalized to unity.

The imperfection of a matrix results in a spread of the ZPL frequencies $v_{0}$, which can be described by the statistical inhomogeneous distribution function (IDF) $N\left(v_{0}\right)$. Depending on the type of matrix defects, this function may have a Gaussian, a Lorentzian [19], or more general Voigt shape [20].

If the inhomogeneous broadening is large, as is the case for amorphous media, the absorption spectrum in the region of an electronic transition consists of a broad band formed by two overlapped bands described by convolutions $\alpha_{1} N^{*} z_{1}$ and $\left(1-\alpha_{1}\right) N^{*} p_{1}$.

Upon irradiation of such an inhomogeneous ensemble of impurity centers by monochromatic light at the frequency $v_{L}$ with the spectral linewidth $\gamma_{L}$ much smaller than the ZPL homogeneous linewidth $\gamma_{1}$, a subsystem of centers is excited whose ZPL frequency distribution is described by a new IDF $M\left(v_{0}-v_{L}\right)[8,9]$. The properties of this ID, namely, the dependence of its shape on the exciting laser frequency and on the irradiation time determine all the features of the monochromatic excitation of luminescence and hole burning.

\subsubsection{Photostable Molecular Systems}

Consider first photostable systems in which no photoinduced transformations occur upon optical excitation. In this case, the IDF of the subsystem of centers selected by the monochromatic light has the form $[8,9]$

$$
\mathbf{M}\left(v_{0}-v_{L}\right)=\mathbf{N}\left(v_{0}\right) \mathbf{A}\left(v_{0}-v_{L}\right) / \mathbf{A}(0)
$$

This DF consists of a narrow resonance peak located at the excitation frequency, and a broad band. The peak is the IDF of subsystem of centers excited resonantly and selectively via the ZPLs. Its width is equal to the homogeneous ZPL width $\gamma_{1}$. The wide band is the IDF of another subsystem of centers excited nonresonantly and nonselectively via broad PSBs. The width of this band is determined by the widths of the IDF $N\left(v_{0}\right)$ and of PSB $\left(1-\alpha_{1}\right) p_{1}$. In particular, for Gaussian IDF and PSB shapes, this width is equal to $\Gamma_{1} /\left(1+n^{2}\right)^{1 / 2}$ (where $\left.n=\Gamma_{1} / \Gamma\right)$ : The shape of the IDF (2) depends strongly on the excitation frequency, the ratio of intensities of the peak and of the broad band and the distance between their maxima being dependent on this frequency $[8,9]$ (for Gaussian shapes of the IDF and PSB, this distance is equal to $\left(n^{2} v_{L}+\Delta_{1}\right) /\left(n^{2}+1\right)$. 
The integrated intensities of the peak and of a broad band of IDF (2) are equal to the number of centers excited selectively and nonselectively, respectively. The ratio of these intensities characterizes the selectivity of the excitation and is equal to [8,9]:

$$
R\left(v_{L}\right)=\frac{\alpha_{1} \int_{-\infty}^{\infty} N\left(v_{0}\right) z_{1}\left(v_{0}-v_{L}\right) d v_{0}}{\left(1-\alpha_{1}\right) \int N\left(v_{0}\right) p_{1}\left(v_{0}-v_{L}+\Delta_{1}\right) d v_{0}}=\frac{\alpha_{1} N\left(v_{L}\right)}{\left(1-\alpha_{1}\right) N * p_{1}}
$$

(In the integration of the convolution in the numerator of (3) and below we consider the ZPL to be a delta function because its width is much less than those of the IDF and PSB).

The dependencies of the excitation-selectivity coefficient (3) on the laser frequency for different shapes of the IDF and PSB are shown in Fig.1(5.2). For Gaussian or Lorentzian shapes of the IDF and PSB this coefficient takes the form [12]:

$$
\begin{aligned}
& R_{G}\left(v_{L}\right)=\frac{\alpha_{1}\left(n^{2}+1\right)^{1 / 2}}{1-\alpha_{1}} \exp \left(\frac{\Delta_{1}^{2}}{\Gamma_{1}^{2}} \ln 2\right) \exp \left[-\left(\frac{v_{L}+\Delta_{1} / n^{2}}{\Gamma\left(1+1 / n^{2}\right)^{1 / 2}}\right) \ln 2\right] \\
& R_{L}\left(v_{L}\right)=\frac{\alpha_{1}(n+1)}{1-\alpha_{1}} \frac{1+\left[\left(v_{L}-\Delta_{1}\right) /\left(\Gamma_{1}+\Gamma\right)\right]^{2}}{1+\left(v_{L} / \Gamma\right)^{2}}
\end{aligned}
$$

The luminescence spectrum observed upon monochromatic excitation is described by a convolution of the IDF (2) with the homogeneous luminescence spectrum (1b):

$$
F\left(v-v_{L}\right)=i N\left(v_{0}\right) A\left(v-v_{L}\right) E\left(v-v_{0}\right) d v_{0}
$$

It follows from (1) that spectrum (5) contains four contributions [18]. The ZPL in (5) is described by the convolution

$$
F_{z z}=\alpha_{1} \alpha_{2}\left(N z_{1}\right)^{*} z_{2}=\alpha_{1} \alpha_{2} N\left(v_{L}\right) z_{1}\left(v_{L}-v_{0}\right) * z_{2}\left(v-v_{0}\right)
$$

while the PSB is the superposition of three contributions:

$$
\begin{aligned}
& W=F_{\mathrm{qp}}+\mathrm{F}_{\mathrm{pz}}+\mathrm{F}_{\mathrm{pp}} \\
& \mathrm{F}_{\mathrm{q}}=\alpha_{1}\left(1-\alpha_{2}\right)\left(N \mathrm{Nz}_{1}\right)^{*} \mathrm{p}_{2}=\alpha_{1}\left(1-\alpha_{2}\right) \mathrm{N}\left(v_{\mathrm{L}}\right) \mathrm{p}_{2}\left(v-v_{\mathrm{L}}+\Delta_{2}\right) \\
& \mathrm{F}_{\mathrm{pz}}=\left(1-\alpha_{1}\right) \alpha_{2}\left(N \mathrm{~Np}_{1}\right)^{*} \mathrm{z}_{2}=\left(1-\alpha_{1}\right) \alpha_{2} \mathrm{~N}(v) \mathrm{p}_{1}\left(v_{L}-v-\Delta_{1}\right), \\
& \mathrm{F}_{\mathrm{pp}}=\left(1-\alpha_{1}\right)\left(1-\alpha_{2}\right) \mathrm{N}(v) \mathrm{p}_{1}\left(v_{\mathrm{L}}-v-\Delta_{1}\right) \mathrm{p}_{2}\left(v-v_{\mathrm{L}}+\Delta_{2}\right) .
\end{aligned}
$$



The contribution $F_{z z}+F_{z p}$ corresponds to impurity centers excited selectively via narrow $Z$ PLs, while $F_{p z}+F_{p p}$ corresponds to centers excited nonselectively through broad PSBs.

The selective contribution virtually coincides with the homogeneous luminescence spectrum (1b), the only difference being that the ZPL $F_{z z} s$ the convolution of ZPLs $z_{1}$ and $\mathbf{z}_{2}$ :

$$
F_{z z}+F_{\mathbf{4}}=\alpha_{1} N\left(v_{L}\right)\left[\alpha_{2} z_{1}^{*} z_{2}+\left(1-\alpha_{2}\right) p_{2}\right]
$$

The relative contribution of the selectively excited spectrum $F_{z z}+F_{z p}$ to the spectrum (5) observed experimentally is equal to $[10,12]$

$$
P\left(v_{L}\right)=\frac{\int_{-\infty}^{\infty}\left(F_{z z}+F_{s p}\right) d v}{\int_{-\infty}^{\infty} F d v}=\frac{R\left(v_{L}\right)}{1+R\left(v_{L}\right)}
$$

Equation (8) also describes the ratio $\alpha_{E} / \alpha_{2}$ of the effective Debye-Waller factor $\alpha_{E}$ (which is determined from the experimental spectrum by measuring the ratio of the integrated intensity of the ZPL $F_{Z z}$ to that of the total spectrum (5)) and the true DebyeWaller factor $\alpha_{2}$ for the homogeneous spectrum (1b).

Equation (8) relates the homogeneous luminescence spectrum (1b) to the experimentally recorded spectrum (5). Eqs. 3, 4, 8 and Fig.1(5.2) illustrate an important result (4) according to which for any impurity system there exists a certain excitation frequency that provides the maximum selectivity of excitation. In this case the shape of the spectrum observed is as close to the true homogeneous spectrum as possible for a given impurity system. In particular, for Gaussian shapes of the IDF and PSB, this frequency is equal to $v_{L}=-\Delta_{1}\left(\Gamma / \Gamma_{1}\right)^{2}$, as follows from (4a).

It follows from the above that the shape of luminescence spectrum (5) strongly depends on the excitation frequency. As $v_{L}$ increases, the relative intensity of the ZPL in (5) decreases, while the PSB W broadens, its shape is changed and its maximum shifts from the ZPL maximum.

Equations (6a) - (6d) show that in the case of Gaussian shapes of the IDF and PSB and a Lorentzian ZPL, the selective contribution $F_{z z}+F_{\text {ap }}$ follows the laser frequency upon its tuning $\left(v_{z z}=v_{L}\right.$ and $v_{y p}=v_{L}-\Delta_{2}$, where $v_{z z}$ and $v_{z p}$ are positions of maxima of $F_{z z}$ and $F_{4 p}$, respectively), while the shifts of bands $F_{p z}$ and $F_{p p}$ are $v_{p z}=\left(v_{L}-\Delta_{1}\right) /\left(n^{2}+1\right)$ and $v_{p p}=v_{L} /\left(n^{2}+1\right)-\left[\Delta_{1} /\left(n^{2}+1\right)+\Delta_{2}\right]$, respectively, i.e. they are shifted by a factor of 
$1 /\left(n^{2}+1\right)$ slower than the laser frequency. This results in a change of the shape of the PSB W upon scanning the laser frequency.

We observed the above dependence of the shape of the luminescence spectrum on the excitation frequency for porphyrins in amorphous matrices excited by a tunable dye laser at helium temperature $[8,9,21,22]$ and quantitatively interpreted it in $[8,9]$. Similar dependencies were observed for luminescence spectra of perylene [18] and anthracene [23] and were qualitatively interpreted in [18, 24].

Note that impurity systems with a strong electron-phonon coupling, whose homogeneous spectra do not exhibit sharp ZPLs but show only broad PSBs because of the low Debye-Waller factor, can also be selectively excited through these bands. The luminescence spectrum observed upon such excitation will be described by equation ( $6 \mathrm{~d}$ ). The half-width of this spectrum will be narrowed to $[10,12]$

$$
\Gamma_{c m}=\left[\left(\Gamma^{-2}+\Gamma_{1}^{-2}\right)^{-1}+\Gamma_{1}^{2}\right]^{1 / 2}
$$

as compared to the half-width $\left(\Gamma^{2}+\Gamma_{1}{ }^{2}\right)^{1 / 2}$ of the luminescence spectrum observed upon broadband excitation (we assume in (9) for simplicity that half-widths $\Gamma_{1}$ and $\Gamma_{2}$ of PSBs $p_{1}$ and $p_{2}$ in (1a) and (1b) are the same).

However, it is obvious that the effect of line narrowing will be less pronounced than upon exciting via sharp ZPLs, so that it is appropriate to call monochromatic excitation through broad PSBs a quasi-selective excitation. A characteristic feature of this excitation is that upon scanning the laser frequency $v_{L}$ across the inhomogeneous absorption band, the selected luminescence spectrum will shift slower than $v_{L}$ in contrast to excitation via ZPLs, the shift of vibronic bands in this spectrum being described by the equation $[10,12]$

$$
v_{e m}=v_{L} /\left(n^{2}+1\right)-\Delta\left(n^{2}+2\right) /\left(n^{2}+1\right)-\Omega
$$

where $\Omega$ is the molecular vibrational mode frequency (we assume in (10) for simplicity that $\Delta_{1}=\Delta_{2}=\Delta$ ).

The slope $1 /\left(\mathrm{n}^{2}+1\right)$ of the linear dependence $v_{c m}\left(v_{L}\right)$ is determined by the ratio $n=\Gamma_{1} / \Gamma$ of half-widths of the PSB and IDF. This ratio in amorphous impurity systems is about 0.5 , corresponding to the slope value of 0.8 , which can be readily experimentally distinguished from a unit slope observed upon selective excitation via ZPLs (in the latter case, the slope $1 /\left(n^{2}+1\right)=1$ because $\left.n=\gamma_{1} / \Gamma \ll 1\right)$. 
Recently, we observed the above effects upon selective excitation of thin films of poly(p-phenylene) via PSBs at room temperature (see Report 1).

We also studied [13] the dependence of the luminescence spectrum shape on the excitation frequency in the most general case for the impurity systems with quasi-local vibrations. These vibrations appear as narrow peaks superimposed on a broad acoustic PSB and thus provide additional channels for the selective excitation. As a result, the spectrum recorded exhibits additional narrow lines that are absent in the true homogeneous luminescence spectrum [22].

\subsubsection{Photosensitive Molecular Systems}

Consider now photosensitive systems, in which the monochromatic excitation of luminescence is accompanied by hole burning $[12,14,16]$. In this case, upon irradiation by a laser at frequency $v_{L}$, the number of impurity molecules with the ZPL maximum at $v_{0}$ will decrease with time as

$$
N\left(v_{0}-v_{L}, t\right)=N\left(v_{0}\right)\left\{C_{1} \exp \left[-\left(C_{1}+C_{2}\right) t\right]+C_{2}\right\} /\left(C_{1}+C_{2}\right)
$$

where $C_{1}=I \sigma_{z} \cdot \varphi A\left(v_{0}-v_{0}\right) / A(0)$ is the rate of hole burning $\left(s^{-1}\right), C_{2}$ is the rate of the reverse reaction, $I$ is the laser power density at the sample (photon $\mathrm{cm}^{-2} \mathrm{~s}^{-1}$ ), $\sigma_{z}$ is the molecular absorption cross section at the ZPL maximum $\left(\mathrm{cm}^{2}\right)$, and $\varphi$ is the quantum yield of the hole burning reaction. The nonstationary IDF (11) was obtained from the kinetic equations for a four-level scheme of hole burning in a quasi-stationary approximation taking into account the reverse reaction.

The nonstationary IDF of a subsystem of luminescent centers excited by a laser at a frequency $n$ is equal to the product of the IDF (11) by the homogeneous absorption spectrum $A\left(v_{0}-v_{L}\right) / A(0)$ :

$$
M\left(v_{0}-v_{L}, t\right)=\frac{N\left(v_{0}\right) A\left(v_{0}-v_{L}\right)}{\left(C_{1}+C_{2}\right) A(0)}\left\{C_{1} \exp \left[-\left(C_{1}+C_{2}\right) t+C_{2}\right]\right\}
$$

The stationary $\mathrm{DF}(2)$ is a particular case of $(12)$ for $C_{1}=C_{2}=0$.

The shape of IDF (12) drastically changes during hole burning, the intensity of its narrow peak rapidly decreases because of resonance burning off centers via narrow ZPLs since the cross section for absorption via ZPLs is very large. Because the burning rate at the peak maximum is much higher than on its wings, the form of the peak changes during the burning and gradually deviates from its initial Lorentzian shape. This is 


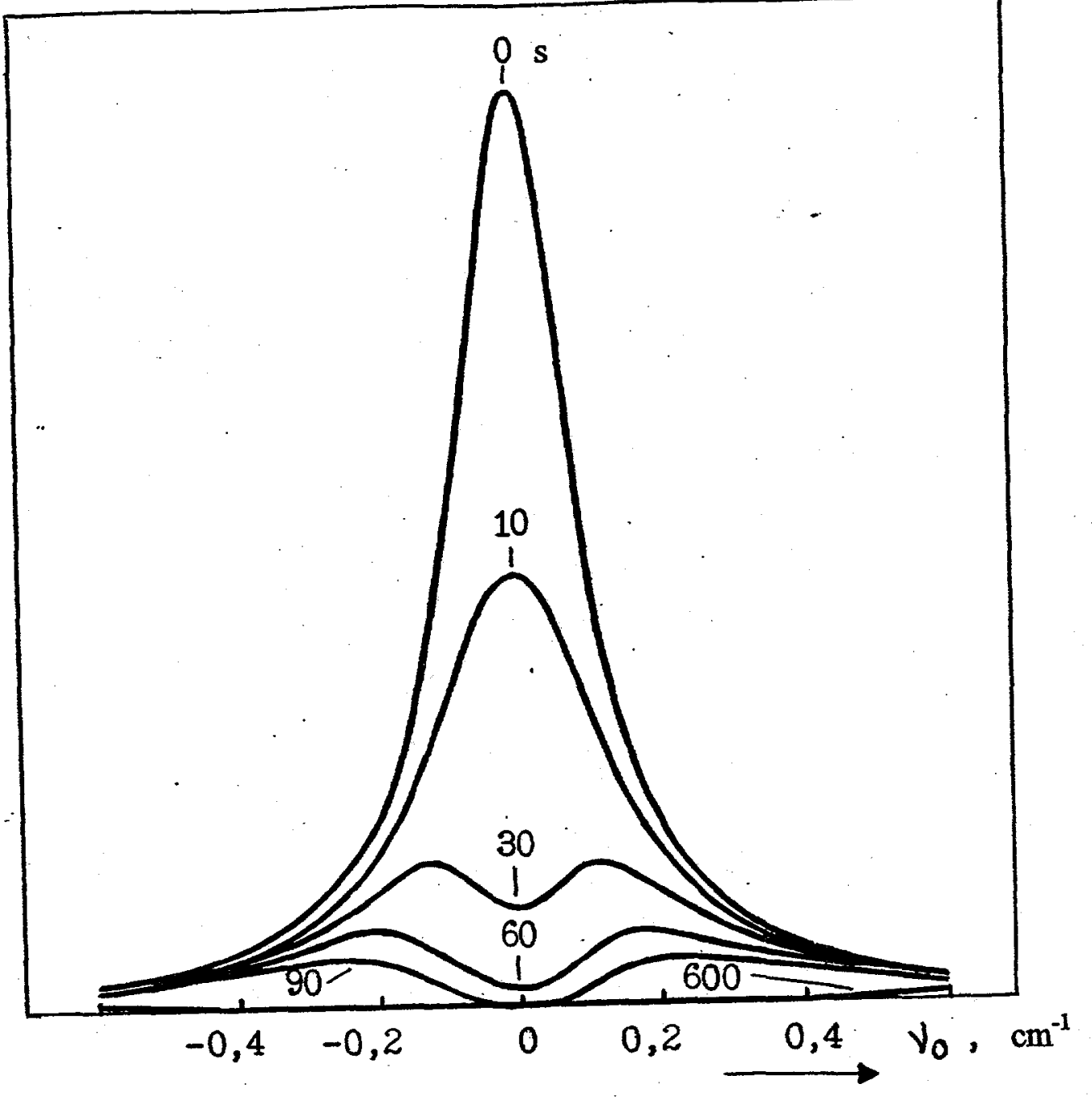

Fig.2(5.2). Hole burning in the peak of IDF (12). The model parameters are as in Fig.1(5.2). $C_{2}=0, I \sigma_{z} \varphi=0.074 \mathrm{~s}^{-1}, v_{L}=0$. The numbers at the curves indicate the burning time in seconds $[12,16]$. 
followed by hole burning in the peak, which splits thus into two peaks, as shown in Fig.2(5.2). By differentiating (12) with respect to $n$, we obtain the time variation of the positions of the two new peaks. For a Lorentzian shape of the ZPL, we have

$$
v_{1,2}=v_{L}+\left(\gamma_{1} / 2\right)\left(I \sigma_{z} \varphi t-1\right)
$$

where $\gamma_{1}$ is the FWHM (full width at half maximum) of the ZPL.

It follows from this equation that burning of a hole in the peak of the IDF (12) begins at $t=1 / 1 \sigma_{z} \varphi$. Thus, it is possible in principle to observe in the luminescence spectrum, which is a convolution of the IDF (12) with the homogeneous spectrum (1b), hole burning in the natural contour of the homogeneous ZPL.

The intensity of the broad band of the IDF (12) corresponding to the nonselectively burnt centers decreases in the course of the burning much slower than the intensity of the peak, because the ratio of the burning rates via ZPL and PSB is proportional to $\sigma_{z} / \sigma_{p}=\Gamma_{1} / \gamma_{1}>100$ for pure electronic transitions in complex molecules at the liquid helium temperature. For this reason the form of the luminescence spectrum changes during the excitation $[14,15]$.

\subsection{Burning Persistent Holes in Absorption Spectra of Molecules and Molecular Aggregates}

\subsubsection{Dependence of the Hole Shape on the Excitation Frequency and Irradiation}

Time (Dose). Relationship between the Hole Shape and the Homogeneous

\section{Absorption Spectrum}

Consider a general picture of hole burning taking into account both selective burning off centers via narrow ZPLs and nonselective burning off other centers from the inhomogeneous ensemble via broad PSBs. The zero-phonon hole properties are discussed in the next section.

If the irradiation of the inhomogeneous ensemble of impurity centers by monochromatic light at frequency $v_{L}$ results in the change of electronic energy of these centers due to some photochemical reaction or their reorientation in a matrix then the DF

of the ZPLs frequencies $v_{0}$ of the subsystem of centers burnt off for a time $t$ has the form [15]

$$
M\left(v_{0}-v_{L}, t\right)=\left[C_{1} /\left(C_{1}+C_{2}\right)\right] N\left(v_{0}\right)\left\{1-\exp \left[-\left(C_{1}+C_{2}\right) t\right]\right\}
$$


The IDF (14) describes the shape of the hole burnt in the initial IDF $N\left(v_{0}\right)$. The shape of the hole in the absorption spectrum is described by the convolution

$$
H\left(v-v_{L}, t\right)=i M\left(v_{0}-v_{L}, t\right) A\left(v-v_{0}\right) d v_{0}
$$

All the properties of the hole: the dependence of its shape, width, and depth on the burning frequency, power, and irradiation time are determined by the dependence of the shape of IDF (14) on these parameters. The properties of this DDF were studied in our papers [11, 15]. The shape of the IDF (14) strongly depends on the burning frequency and on the irradiation dose It.

The resonance peak of IDF (14) at the burning frequency $n$ describes the subsystem of centers burnt selectively via ZPLs, whereas the broad band corresponds to another subsystem of centers burnt nonselectively via PSBs. At a low irradiation dose, when It $\ll 1 / \sigma_{z} \varphi$, the shape of IDF (14) coincides with that of stationary IDF (2).

The hole-burning selectivity is determined by the ratio of numbers of selectively burnt centers and centers burnt nonselectively, i.e. by the ratio of the integrated intensities of the peak and broad band. This ratio decreases upon burning, i.e.the burning selectivity decreases. The reason is that burning via ZPLs occurs much more rapidly than via PSBs, so that the peak of IDF (14) saturates rapidly, while the broad band continues to increase. Thus, in the absence of the reverse reaction $\left(C_{2}=0\right)$, virtually the entire inhomogeneous ensemble of centers will be burnt off after some time, whereas in the presence of the reverse reaction $\operatorname{IDF}(14)$ will stabilize at $N\left(v_{0}\right) C_{1} /\left(C_{1}+C_{2}\right)$.

To calculate the form of hole (15) as a function of the burning frequency and of the irradiation time, we expand the exponential in (14) (at $\mathrm{C}_{2}=0$ ) in a series, neglecting the cross terms of the type $z_{1}{ }^{m-1} p_{1}{ }^{k}(k=1,2,3, \ldots m-1)$ [25]. Then IDF (14) can be approximately written in the form $M=M_{z}+M_{p}$, where

$$
M_{Z}\left(v_{0}-v_{L}, t\right)=N\left(v_{0}\right)\left\{1-\exp \left[-\beta \operatorname{ta} \alpha_{1} z_{1}\left(v_{0}-v_{L}\right)\right]\right\}
$$

and

$$
M_{p}\left(v_{0}-v_{L}, t\right)=N\left(v_{0}\right)\left\{1-\exp \left[-\beta t\left(1-\alpha_{1}\right) p_{1}\left(v_{0}-v_{L}+\Delta_{1}\right)\right]\right\}
$$

are IDFs of the subsystems of centers burnt selectively and nonselectively, respectively $\left(\beta=I \sigma_{z} \varphi / A(0)\right)$. 
The nonstationary coefficient of hole-buming selectivity is equal to the ratio of integrals of (16a) and (16b). At a low irradiation dose, when It $\ll 1 / \sigma_{z} \varphi$, this coefficient coincides with the luminescence-excitation selectivity coefficient (3).

It follows from (15) and (16) that the hole spectrum contains four contributions and consists of the zero-phonon hole

$H_{z L}\left(v-v_{L}, t\right)=\alpha_{1} \int_{-\infty}^{\infty} N\left(v_{0}\right)\left\{1-\exp \left[-\beta t \alpha_{1} z_{i}\left(v_{0}-v_{L}\right)\right]\right\} z_{1}\left(v-v_{0}\right) d v_{0}$

located on a broad pedestal made up of the superposition of three phonon holes:

$$
\begin{aligned}
& \mathrm{H}_{\mathbf{p}}\left(v-v_{\mathrm{L}}, \mathrm{t}\right)=\left(1-\alpha_{1}\right) \int_{-\infty}^{\infty} \mathrm{N}\left(v_{0}\right)\left\{1-\exp \left[-\beta \mathrm{t} \alpha_{1} z_{1}\left(v-v_{0}\right)\right]\right\} \mathrm{x} \\
& \operatorname{xp}_{1}\left(v-v_{0}-\Delta_{1}\right) \mathrm{d} v_{0} \\
& \mathrm{H}_{\mathrm{pz}}\left(v-v_{\mathrm{L}}, t\right)=\alpha_{1} \mathrm{~N}\left(v_{0}\right)\left\{1-\exp \left[-\beta \mathrm{t}\left(1-\alpha_{1}\right) \mathrm{p}_{1}\left(v-v_{\mathrm{L}}+\Delta_{\mathrm{l}}\right)\right]\right\} \\
& \mathrm{H}_{\mathrm{pp}}\left(v-v_{\mathrm{L}}, \mathrm{t}\right)=\left(1-\alpha_{1}\right) \int_{-\infty}^{\infty} \mathrm{N}\left(v_{0}\right)\left\{1-\exp \left[-\beta \mathrm{t}\left(1-\alpha_{1}\right) \mathrm{p}_{1}\left(v_{0}-v_{\mathrm{L}}+\Delta_{1}\right)\right]\right\} \mathrm{x} \\
& \operatorname{xp}_{1}\left(v-v_{0}-\Delta_{1}\right) \mathrm{d} v_{0}
\end{aligned}
$$

For low irradiation doses, the zero-phonon hole (17a) $H_{z z}=\alpha_{1}^{2} N\left(v_{L}\right) \beta t z_{1} * z_{1}$, therefore its width is twice the width $\gamma_{1}$ of the ZPL $z_{1}$ if the ZPL is a Lorentzian, or equal to $2^{1 / 2} \gamma_{1}$, if the ZPL is a Gaussian.

For low irradiation doses, the spectrum of the selectively burnt hole is

$$
\mathrm{H}_{z z}+\mathrm{H}_{\mathrm{zp}}=\alpha_{1} \beta \mathrm{tN}\left(v_{\mathrm{L}}\right)\left[\alpha_{1} \mathrm{z}_{1}^{*} \mathrm{z}_{1}+\left(1-\alpha_{1}\right) \mathrm{p}_{1}\right]
$$

i.e., it virtually coincides with the homogeneous absorption spectrum, except that the ZPL width is double the width of the homogeneous ZPL in the absorption spectrum. The relative contribution of a selectively burnt hole (18) to the total spectrum of the hole (15) is described by function ( 8 ) which relates the homogeneous absorption spectrum to the hole spectrum.

Fig.1(5.3) shows the dependence of the hole shape on the burning time (dose) and burning frequency. 


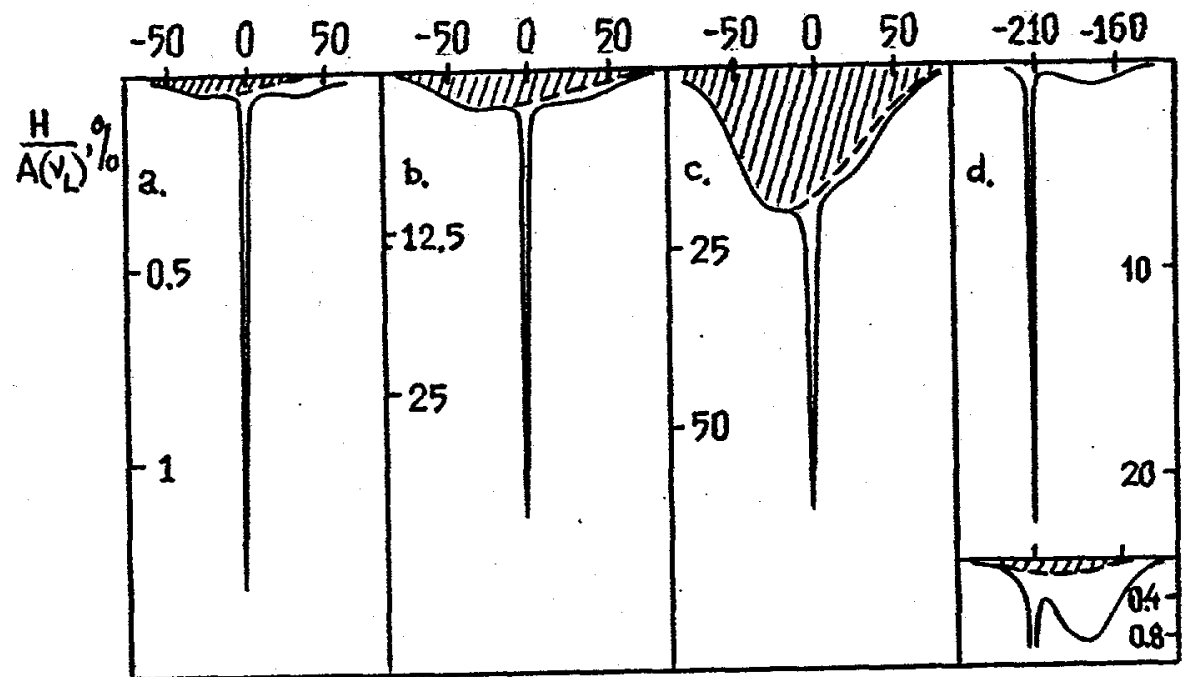

Fig.1(5.3). Dependence of the hole shape on the irradiation time (dose) and burning frequency. Model parameters are: ZPL $\alpha_{1} z_{1}$ is a Lorentzian with FWHM $0.3 \mathrm{~cm}^{-1}$, PSB $\left(1-\alpha_{1}\right) p_{1}$ and IDF N(vo) are Gaussians with FWHMs 40 and $100 \mathrm{~cm}^{-1}$, respectively, $\Delta_{1}=34 \mathrm{~cm}^{-1}, \alpha_{1}=0.42, I \sigma_{z} \varphi=0.052 \mathrm{~s}^{-1}, C_{2}=0 .(\mathrm{a}, \mathrm{b}, \mathrm{c})$ Holes burnt at the IDF maximum $\left(v_{\mathrm{L}}=0\right)$ for $1 \mathrm{~s}(\mathrm{kt}=0.052), 60 \mathrm{~s}(\mathrm{kt}=3.12)$ and $600 \mathrm{~s}(\mathrm{kt}=31.2)$, respectively. Hatched area corresponds to the nonselective contribution $\mathrm{H}_{\mathrm{pz}}+\mathbf{H}_{\mathrm{pp}}$; (d) hole burnt at $v_{\mathrm{L}}=-212.5 \mathrm{~cm}^{-1}$, when the hole burning selectivity reaches a maximum; the inset shows a small nonselective contribution (the hatched area) to the phonon pedestal $[26,27]$. 


\subsubsection{Dependence of the Zero-Phonon Hole Shape, Width, Area, and}

\section{Depth on the Irradiation Time (Dose)}

The zero-phonon hole broadens and changes its shape during hole burning because of gradual burning off impurity centers via ZPLwings and saturation. Therefore, to determine correctly the hole width, one has to take into account this effect by extrapolating the hole width to the zero burning time. Various aspects of the zero-phonon hole burning kinetics have been studied by many authors (see references in [26]). However, it was suggested in these papers that the burning laser linewidth is far smaller than the homogeneous ZPL linewidth in absorption, and hence in calculations the laser line was considered as a delta function. For the allowed electronic transitions in organic molecules whose linewidths can be as small as $10^{-3}-10^{-4} \mathrm{~cm}^{-1}$, this approximation is valid only for the single-frequency lasers having very narrow linewidths of the order of $1 \mathrm{Mhz}\left(310^{-3} \mathrm{~cm}^{-1}\right)$. However, lasers with linewidths in the range from 0.01 to $1 \mathrm{~cm}^{-1}$ are often used in experiments. Therefore, one has to study kinetics of hole buming for various relations between laser and ZPL linewidths.

The zero-phonon hole spectrum is described by the convolution of the homogeneous ZPL $\alpha_{1} Z_{1}\left(v-v_{0}\right)$ with the IDF $M_{2}\left(v_{0}-v_{L}, t\right)$ of the ZPL frequencies of the subsystem of centers burnt off selectively via ZPLs for a time $t$ :

$$
H_{z}\left(v-v_{L}, t\right)=\alpha_{1} \int_{-\infty}^{\infty} M_{z}\left(v_{0}-v_{L}, t\right) z_{1}\left(v-v_{0}\right) d v_{0}
$$

where the IDF is

$M_{Z}\left(v_{0}-v_{L}, t\right)=N\left(v_{0}\right)\left\{1-\exp \left[-\alpha_{1} \sigma \varphi t \int_{-\infty}^{\infty} L\left(v^{\prime}-v_{L}\right) z_{1}\left(v_{0}-v^{\prime}\right) d v^{\prime}\right]\right\}$

Here, $L\left(v^{t}-v_{L}\right)$ is the laser line contour with a maximum at $v_{L} ; \sigma$ is the integrated cross section for the homogeneous spectrum. Expressions (19) and (20) are valid for systems without reverse reactions.

The kinetics of hole burning is determined by the time dependence of IDF (20) . Consider this dependence. In the most general case, shapes of the laser line and ZPL can be described by Voigt profiles. Therefore, the convolution integral in the exponential in (20) is also can be described by the Voigt function [28]. In practice, the laser line shape is close to a Gaussian, whereas the ZPL shape is a Lorentzian. Therefore, we shall consider 
namely this case without loss of generality. Consider three different relations between widths of the laser line and homogeneous ZPL encountered in experiments.

(a) The laser linewidth is far smaller than the ZPL width $(\delta<<\gamma)$

In this case one can treat the laser line as a delta function. Then IDF (20) takes the form

$$
\mathrm{M}_{\mathrm{ZL}}\left(v_{0}-v_{\mathrm{L}}\right) \sim 1-\exp \left[-\frac{k t}{1+\left(v_{0}-v_{L}\right)^{2} / \gamma^{2}}\right]
$$

where $\mathrm{k}=\mathrm{I} \sigma \varphi \alpha / \pi \mathrm{g}=\mathrm{I} \sigma \varphi$ is the rate of hole burning. It is easy to show that the half-width (HWHM) of contour (21) increases with time as

$$
\Gamma_{L}=\gamma\left[\frac{k t}{\ln \{2 /[1+\exp (-k t)]\}}-1\right]^{1 / 2}
$$

Curve (22) has a weak inflection which is difficult to observe in experiments. The broadening (22) is well described by the approximate expressions

$$
\Gamma_{\mathrm{L}}=\gamma(1+0.3 \mathrm{kt}) \quad \text { at } \mathrm{kt}<6
$$

and

$$
\Gamma_{\mathrm{L}}=\gamma(\mathrm{kt} / \mathrm{ln} 2-1) \quad \text { at } \mathrm{kt}>4
$$

The shape of (21) gradually deviates from an initial Lorentzian during hole burning. This deviation is shown in Fig. 2a(5.3) where contours (21) are presented for different kt. One can see that as kt increases, the intensity of the contour wings begins to fall more rapidly than for a Lorentzian, whereas the intensity at the contour center exceeds that of a Lorentzian.

Contours (21) can be approximated for different kt by different Voigt functions, whose Lorentzian and Gaussian components of width were calculated in [26].

The area under the contour (21) is described by the function

$$
A(k t)=2 p g f(x)
$$




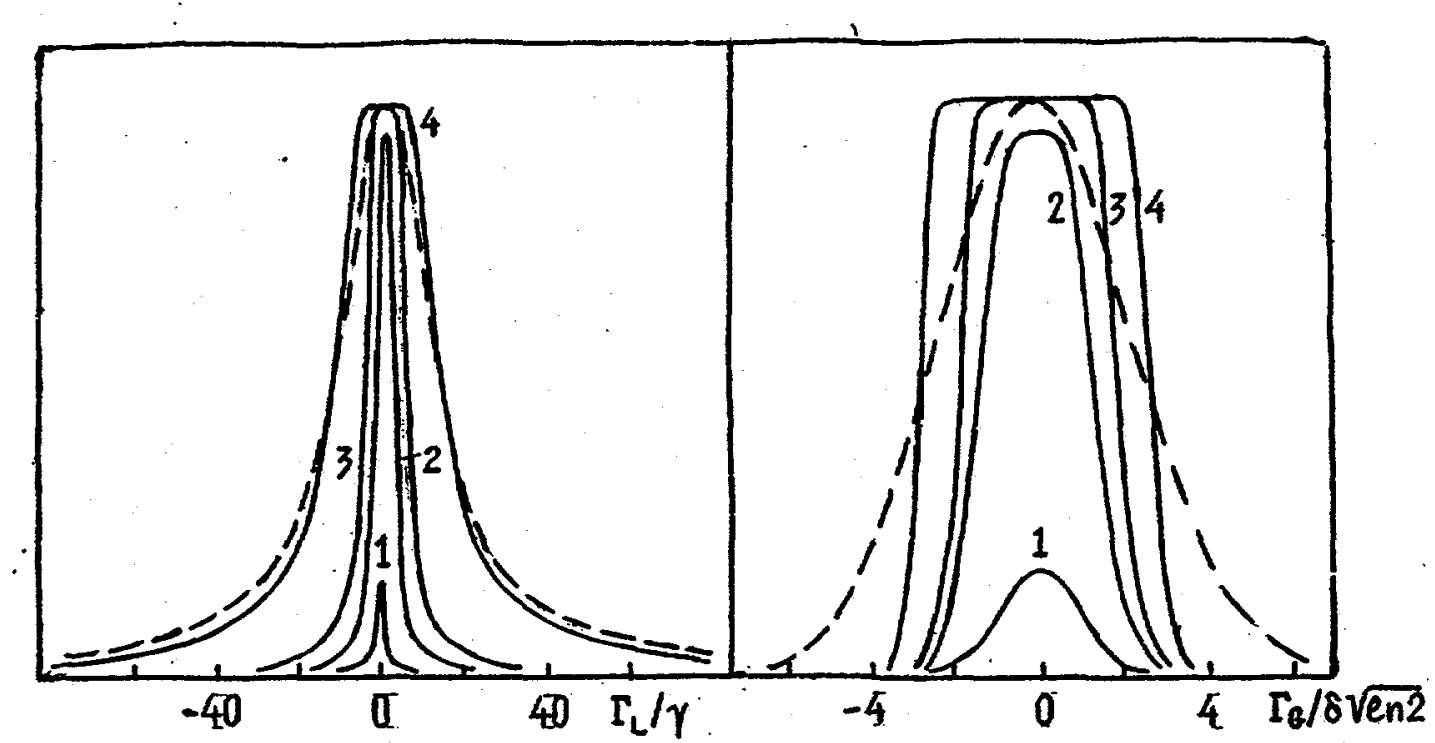

Fig.2(5.3). Dependence of the shape of (a) IDF (21) and (b) (25) on the irradiation dose kt. Shapes 1, 2, 3, 4 correspond to doses $k t=0.2,3,10$ and 100, respectively. Dashed lines show a Lorentzian (Fig. 2a) and a Gaussian (Fig. 2b) with half-widths equal to that of the corresponding IDF at $\mathbf{k t}=100$.

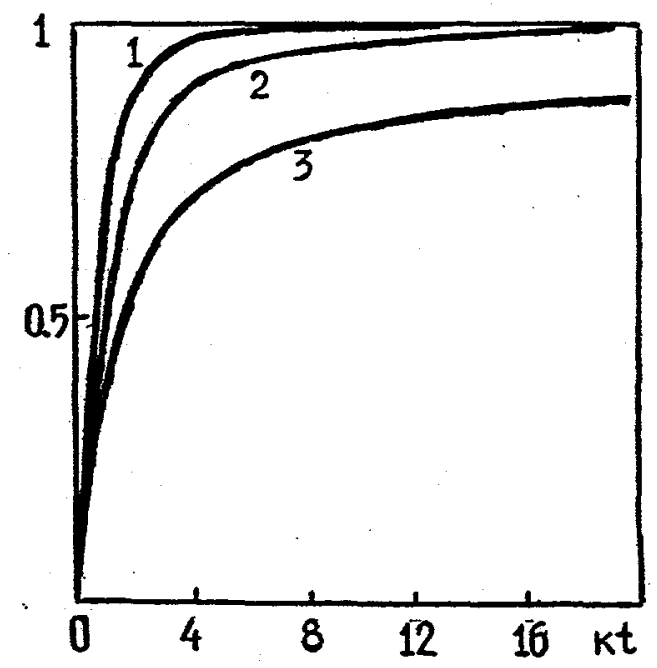

Fig.3(5.3). Kinetics of the hole depth growth: (1) hole in the IDF, (2 and 3) holes in the absorption spectrum in the cases of Gaussian and Lorentzian ZPL shapes, respectively, when the laser line is considerably narrower than the ZPL. 
considered in [30], where $f(x)=x \exp (-x)[J(x)+J(x)], x=k t / 2 ; J(x)$ and $J(x)$ are modified Bessel functions of zeroth and first order, respectively. For small kt $<1$, one can use the linear approximation $A(k t)=\pi \gamma k t$, and for large $k t>20, A(k t)=2(\pi k t)^{1 / 2} \gamma$.

(b) The laser linewidth is far greater than the ZPL width $(\delta>>\gamma)$ In this case the $\mathrm{DF}(20)$ takes the form

$$
M_{z G}\left(v_{0}-v_{L}, t\right) \sim 1-\exp \left\{-k t \exp \left[-\left(v_{0}-v_{L}\right)^{2} / \delta^{2}\right]\right\}
$$

where $k=I \sigma \varphi \alpha / \pi^{1 / 2} \delta$. The HWHM of contour (25) is equal to

$$
\Gamma_{G}=\delta\left[\ln \frac{k t}{\ln [2 /\{1+\exp (-k t)\}]}\right]^{1 / 2}
$$

The broadening (26) is well approximated by equations

$$
\Gamma_{\mathrm{G}}=\delta(\ln 2)^{1 / 2}(1+0.18 \mathrm{kt}) \quad \text { at } \mathrm{kt}<2
$$

and

$$
\Gamma_{\mathrm{G}}=\delta[\ln (\mathrm{kt} / \ln 2)] \quad \text { at } k t>3 .
$$

Upon hole burning, the shape of (25) deviates gradually from an initial Gaussian, its wings being steeper, while at the line center the intensity, on the contrary, becomes higher than for a Gaussian. For kt $\rightarrow \infty$, contour (25) tends to a rectangular one (Fig. $2 \mathrm{~b}(5.3))$.

The area under contour (25) is well approximated in the $0<\mathbf{k t}<30$ region by a simple expression [30]

$$
A(k t)=(\pi)^{1 / 2} \delta k t \exp \left[-(k t)^{1 / 2} / 2\right]
$$

(c) The laser linewidth is comparable with the ZPL width $(\delta \sim \gamma)$ In this case the IDF (20) has the form

$$
M_{z V}\left(v_{0}-v_{L}, t\right) \sim 1-\exp \{-\operatorname{ktF}(a, x)\}
$$


where $F(a, x)$ is the Voigt function [31] and $k=I \sigma \varphi \alpha /(\pi)^{1 / 2} \delta$.

The HWHM $\Gamma_{v}$ of contour (29) is determined from the equation

$$
F\left(a, \Gamma_{V}\right)=(1 / k t) \ln \frac{2}{1+\exp [-k t F(a, 0)]}
$$

using the tables for Voigt functions [31]. The dependence of the area under curve (29) is described by the "curve of growth" [30].

Consider now a change in the hole shape (19) in the absorption spectrum in the process of hole burning. When the laser line is much narrower than the homogeneous ZPL, the hole shapes (19) at various $k t$ are described by Voigt functions that are the convolutions of the ZPL shape with Voigt approximations of hole shapes in the IDF.

The hole depth is described by the function

$$
H_{s z}(0, t)=\int_{-\infty}^{\infty}\left\{1-\exp \left[-\frac{k t}{1+\left(v_{0}-v_{L}\right) / \gamma^{2}}\right]\right\} \frac{d v_{0}}{1+\left(v_{0}-v_{L}\right)^{2} \gamma^{2}}
$$

The integral can be easily calculated [33]:

$$
\mathrm{H}_{\mathrm{zz}}(0, \mathrm{t})=1-\exp (-\mathrm{kt} / 2) \mathrm{I}_{0}(\mathrm{kt} / 2)
$$

Function (32) is shown in Fig.3(5.3) (curve 3) together with the function 1 - $\exp (-$ kt) that describes the depth of the hole burnt in the IDF. One can see that the hole in the absorption spectrum is saturated much more slowly than exponentially. For comparisonthe figure shows also the time dependence of the hole depth in the case of a Gaussian ZPL shape calculated from data of [33].

If the laser line is much broader than the homogeneous ZPL, the kinetics of hole burning in the absorption spectrum coincides with the kinetics of hole burning in the IDF.

In the case of comparable laser and ZPL linewidths, the broadening of hole in the absorption spectrum can be approximately obtained by a simple shift, along the ordinate axis, of the curve of hole broadening in the $\operatorname{IDF}[26,27]$.

Finally, note that all the results for the hole area growth obtained above for the IDF are also valid for the area of a hole in the absorption spectrum, since these areas are equal, as follows from (19) because of the normalization of the integral of $z\left(v-v_{0}\right)$ to unity. 


\section{Conclusions}

We have presented our model calculations of shapes of selectively excited luminescence spectra and persistent holes burnt by monochromatic laser radiation in inhomogeneous absorption spectra of impurity molecules in amorphous matrices. All the features of selective luminescence excitation and hole burning were shown to be determined by the properties of the IDF of the excited subsystems of the centers selected by the monochromatic light from the total inhomogeneous ensemble of impurity centers. It was shown that the IDF shape strongly depends on the excitation frequency and on the irradiation time, which results in a strong dependence of the luminescence and hole spectra on these parameters. Analytic expressions were obtained for the luminescence-excitation and hole-burning selectivity coefficients, as well as expressions relating homogeneous luminescence and absorption spectra with selectively excited huminescence and hole spectra, respectively. It was shown that for each impurity system there exists a definite excitation frequency at which the excitation selectivity reaches a maximum and, therefore, the experimental luminescence (hole) spectrum is most close to the homogeneous luminescence (absorption) spectrum. The results of our model calculations

were confirmed by our studies of porphyrins, cyanine dyes, J-aggregates of cyanine dyes, and polymers in solutions and thin films.

\section{References}

1. Denisov, Yu.V. and Kizel', V.A. (1967), Opt. Spektrosk. 23, 472.

2. Szabo, A (1970), Phys. Rev. Lett. 25, 924.

3. Kharlamov, B.M., Personov, R.I, and Bykovskaya, L.A. (1974), Opt. Commun. 12, 191.

4. Gorokhovskii, Kaarli, RK, and Rebane, L.A. (1974), Pis'ma Zh. Eksp. Teor. Fiz. 20, 474.

5. Kane, E.O. (1960), Phys. Rev. 119, 40.

6. Krivoglaz, M.A. (1964), Fiz. Tverd. Tela (Leningrad) 6, 1707.

7. Sapozhnikov, M.N., Phys. Status Solidi B (1976) 75, 11.

8. Sapozhnikov, M.N. and Alekseev, V.I. (1984), Chem. Phys. Lett. 107, 265.

9. Sapozhnikov, M.N. and Alekseev, V.I. (1984), Dokl. Akad. Nauk SSSR 279, 358.

10. Sapozhnikov, M.N. (1986), Dokl.Akad. Nauk SSSR 287, 839.

11. Sapozhnikov, M.N. (1986), Fiz. Tverd. Tela 28, 1904.

12. Sapozhnikov, M.N. (1986), Opt. Spektrosk. 61, 331.

13. Sapozhnikov, M.N. (1987), Kratk. Soobshch. Fiz 2, 3.

14. Sapozhnikov, M.N. (1987), Kratk. Soobshch. Fiz. 2, 7. 
(1) Preparation and investigation of high-quality vacuum deposited PPP films on different substrates (dielectrics and semiconductors);

(2) optical studies of electronic excitations in J-aggregates by techniques of steady-state fluorescence spectroscopy, selective laser spectroscopy, and time-resolved spectroscopy;

(3) preparation and study of conjugated polymers mixed with fullerene $C_{60}$ and polyacetylene;

(4) the modern aspects of the selective laser spectroscopy of molecules and molecular aggregates in disordered molecular systems.

\section{The main results are as follows:}

(1) Procedures of preparation of high-quality vacuum deposited PPP films on different substrates (ITO, Si, GaAs and etc.) were developed. Dependences of the structure and optical properties on the conditions of preparation were investigated;

(2) Conditions of preparation of samples giving the high photoluminescence quantum yield were found. The fluorescence lifetimes and spectra of highly oriented vacuum deposited PPP films were studied as a function of the degree of polymerization. The maximum fluorescence yield was achieved for the mean chain length of appoximately 35 monomer units;

(3) Time-resolved fluorescence of initial materials and polymer films on different substrates was measured. For high-quality films, the fluorescence decay times were estimated to be: for PPP on a quartz $t_{1}=1.1 \mathrm{~ns}$, two-exponential decay on GaAs and Si $t_{1}=1.0 \mathrm{~ns}$ and $t_{2}=4.1 \mathrm{~ns}$ and on $A u, t_{1}=1.0 \mathrm{~ns}$ and $t_{2}=2.1 \mathrm{~ns}$.

(4) The selective excitation of luminescence PPP thin films was performed at the temperature range from 5 to $300 \mathrm{~K}$. The total intensity of luminescence monotonically decreases with decreasing temperature and the additional vibronic structure appears at $\mathbf{T}$ $<200 \mathrm{~K}$ instead of a smooth red shoulder observed at room temperature. Spectral positions of peaks are virtually independent of the temperature and the dependence of $v_{\mathrm{cm}}$ on $v_{\text {ex }}$ at $10 \mathrm{~K}$ differs only slightly from that at room temperature.

(5) The dynamics of photoexcitations in different types of J-aggregates of the carbocyanine dye was studied at different temperatures in frozen matrices. The temperature dependent process of phototransformation of one type of J-aggregate to another was discovered. The related potential barrier is from 20 to $30 \mathrm{~cm}^{-1}$ in height.

(6). The temperature dependences of the fluorescence linewidth and of the Urbach tail suggest that the static disorder disappears during exciton relaxation. The Stokes shift seems to be determined completely by exciton-phonon relaxation (dressing) resulting in the self-trapped state. 
(7) For quasi-one-dimensional J-aggregates, we found the systems where the fluorescence spectra are determined either completely by intrasegment processes (localization) or by both intrasegment and intersegment (energy transfer) processes. The optical properties of relatively simple J-aggregates with pure intrasegment relaxation may clarify the problem of the relationship between intrasegment and itersegment processes in the formation of luminescent states in more complicated conjugated polymers.

(8) Conditions of preparation of highly crystalline fullerene $C_{60}$ films by the method of vacuum deposition were found.

(9) Composites of $\mathrm{C}_{60}$ with conjugated polymers PPV and polyacetylene (PA) were prepared. Preliminary results on fluorescence quenching, IR and resonant Raman spectroscopy are consistent with earlier reported ultrafast photoinduced electron transfer from PPV to $\mathrm{C}_{60}$ and show that the electron transfer is absent in the case of the PA - $\mathrm{C}_{60}$ composition.

(10) Strong quenching of PPV fluorescence was observed in the PPV - PA blends. The electron transfer from PPV to PA can be considered as one of the possible mechanisms of the quenching.

(11) Model calculations of shapes of selectively excited luminescence spectra and persistent holes burnt by monochromatic laser radiation in inhomogeneous absorption spectra of molecules and molecular aggregates in amorphous matrices are presented.

The spectral band shapes strongly depend on the excitation frequency and on the irradiation time. The results of these calculations were used for adequate interpretation of selective spectroscopy data obtained for polymer films and J-aggregates in this Report (Parts 1 and 2).

\section{New Proposals}

We are planning to:

: Continue studying the nature of electronic excitations in the linear molecular systems;

developing the methods of preparation of new mixed vacuum deposited highly oriented films of conjugated polymers on various substrates.

In particular, we plan to study:

(1) Optimum conditions of deposition of conjugated polymer films on semiconductor and metal substrates and the time-resolved fluorescence parameters for these systems;

(2) optical properties of sandwiches and mixed structures of conjugated polymer/ $/ \mathrm{C}_{60}$ and of conjugated polymer/conjugated polymer; 
(3) preparation conditions and fluorescent properties of polymer thin films containing dye molecules and J-aggregates;

(4) energy transfer and spectral diffusion in thin films containing conjugated polymers and $\mathrm{J}$-aggegares by the techniques of persistent hole burning and fluorescence line narrowing;

(5) luminescent parameters of the PPP film at elevated temperatures;

(6) transient picosecond photoconductivity of conjugated polymers using the Auston microstripline technique;

(7) the dependence of fluorescence properties of molecular J-aggregates on the chain (segment) length which can be controlled either by the preparation procedure or by laser irradiation;

(8) the properties of polymer film/LB film, polymer film/semiconductor, and polymer film/metal interfaces; and

(9) perform computer simulations of luminescence characteristics of linear molecular systems (polymers and J-aggregates)

In addition, we plan also use techniques of photoinduced and electroinduced spectroscopy, and study the electron-hole recombination in polymer films upon alphaparticle excitation for monitoring of electroluminescence.

\section{Acknowledgments}

The authors thank Profs.V.M.Agranovich, E.L.Frankevich, L.V.Keldysh, Sh.Mukamel and R.Silbey for useful discussions.

\section{List of Researchers:}

1.Dr. S.A. Arnautov

2.Mr. A.S. Averjushkin

3.Mr. M.A. Drobizhev

4.Mrs. N.V.Jidkova

5.Mrs.T.G. Kaplanova

6.Dr. N.V. Kiriakova

7.Prof. V.V. Klyachkovskaya

8. Prof. V.M. Kobryanskii

17. Mr. I.G. Scheblykin
9. Mrs. S.L. Kotova

10. Dr. E.M. Nechvolodova

11. Dr. K.A. Ryjkova

12. Prof. M.N. Sapozhnikov

13. Dr. M.I. Sluch

14. Dr. O.P. Varnavsky

15. Mr. D.M. Basko

16. Mr. A.V. Pimenov 
I N T E R O F F I C E M E MOR A N D U M

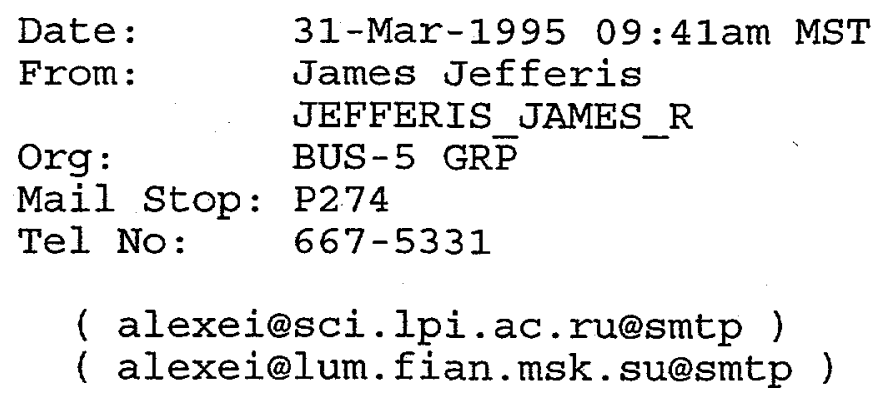

Subject: Agreement 898900004-35, Task Order 001

Dear Dr. Vitukhnovsky:

The first report under task order 001 has been received. In order to receive payment for this effort, please prepare an invoice in accordance with paragraph 1.B.(2) of SECTION F - SPECIAI PROVISIONS, of the Master Task Ordering Agreement. The invoice may be submitted by letter, fax, or e-mail.

Should you have any questions, please contact me. 